\title{
Formation and dynamics of coherent structures involving phase-space vortices in plasmas
}

\author{
B. Eliasson*, P.K. Shukla \\ Institut für Theoretische Physik IV, Fakultät für Physik und Astronomie, Ruhr-Universität Bochum, D-44780 Bochum, Germany
}

Accepted 13 October 2005

Available online 29 November 2005

editor: S. Cowley

\begin{abstract}
We present a comprehensive review of recent theoretical and numerical studies of the formation and dynamics of electron and ion holes in a collisionless plasma. The electron hole is characterized by a localized positive potential in which a population of electrons is trapped, and a depletion of the electron number density, while the ion hole is associated with localized negative potential in which a population of ions is trapped and a depletion of both the ion and electron densities occur. We present conditions for the existence of quasi-stationary electron and ion holes in unmagnetized and magnetized electron-ion plasmas, as well as in an unmagnetized pair-ion plasma. An interesting aspect is the dynamic interactions between ion and electron holes and the surrounding plasma. The dynamics is investigated by means of numerical simulations in which analytical solutions of quasistationary electron and ion holes are used as initial conditions. Our Vlasov simulations of two colliding ion holes reveal the acceleration of electrons and a modification of the initially Maxwellian electron distribution function by the ion hole potentials, which work as barriers for the electrons. A secondary effect is the excitation of high-frequency plasma waves by the streams of electrons. On the other hand, we find that electron holes show an interesting dynamics in the presence of mobile ions. Since electron holes are associated with a positive electrostatic potential, they repel positively charged ions. Numerical simulations of electron holes in a plasma with mobile ions exhibit that the electron holes are, in general, attracted by ion density maxima and repelled by ion density minima. Therefore, standing electron holes can be accelerated by the self-created ion cavities. In a pair plasma, both the temperatures and masses of the positively and negatively charged particles are assumed equal, and therefore one must treat both species with a kinetic theory on an equal footing. Accordingly, a phase-space hole in one of the species is associated with reflected particles in the opposite species. Here standing solitary large-amplitude holes are not allowed, but they must have a propagation speed close to the thermal speed of the particles. We discuss extensions of the existing non-relativistic theories for the electron and ion holes in two directions. First, we describe a fully nonlinear kinetic theory for relativistic electron holes (REHs) in an unmagnetized plasma, and show that the REHs have amplitudes and widths much larger than their non-relativistic counterparts. Second, we extend the weakly nonlinear theories for the electron and ion holes to include the effects of the external magnetic field and the plasma inhomogeneity. The presence of the external magnetic field gives rise to the electron and ion hole structures which have differential scale sizes along and across the magnetic field direction. Consideration of the density inhomogeneity in a magnetized plasma with non-isothermal electron distribution function provides the possibility of bipolar electrostatic pulses that move with the electron diamagnetic drift across the density inhomogeneity and the magnetic field directions. In a plasma with sheared magnetic field we can have fast reconnection involving phase-space vortices in the lower-hybrid frequency range. We further present theoretical and numerical investigations of the nonlinear interaction between ion and electron holes and large amplitude highfrequency electrostatic and electromagnetic waves in plasmas. Since the ion hole is associated with a depletion of both the electron
\end{abstract}

\footnotetext{
* Corresponding author. Tel.: +49 23432 23729; fax: +4923432 14177.

E-mail address: bengt@tp4.rub.de (B. Eliasson).
} 
and ion densities, it can work as a resonance cavity for trapped Langmuir waves whose frequencies are below the electron plasma frequency of the unperturbed plasma. Large-amplitude Langmuir waves can modify the ion hole due to the ponderomotive force which pushes the electrons away from the center of the hole. This process can be modeled by a coupled nonlinear Schrödinger and Poisson system of equations, which exhibit a discrete set of trapped Langmuir eigenmodes in the ion hole in plasmas without and with high- $Z$ charged impurities. In a kinetic description, however, the trapped Langmuir waves are Landau damped due to the relatively small length scale of the ion hole. Intense electromagnetic waves (IEMWs) can also be trapped by the REHs in an unmagnetized plasma. We study the localization of arbitrary large amplitudes IEMWs by incorporating nonlinearities associated with relativistic electron mass increase, relativistic ponderomotive force of the IEMWs, and non-isothermal electron distributions. We find that the combined effects of these three nonlinearities produce extremely large amplitude REHs which traps localized IEMWs. Such a nonlinear structure can accelerate electrons to very high energies. The relevance of our investigation to numerous localized structures observed in laboratory and space plasmas is discussed. We also propose to conduct new experiments that can confirm our new theoretical and simulation models dealing with kinetic nonlinear structures in electron-ion and electron-positron plasmas. Finally, we present our views of possible extensions of theoretical models and computer simulations that have been reviewed in here.

(C) 2005 Elsevier B.V. All rights reserved.

\section{PACS: 52.35.Mw; 52.35.Ra; 94.30.Tz}

Keywords: Electron holes; Ion holes; Phase-space vortices; Double layers; Pair plasmas; Electromagnetic waves

\section{Contents}

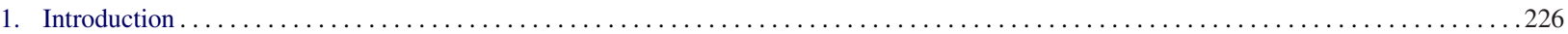

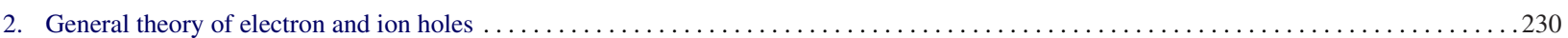

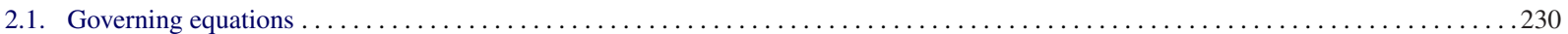

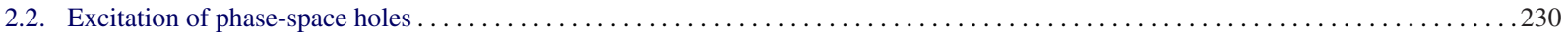

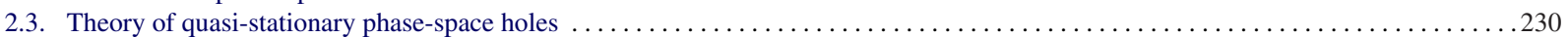

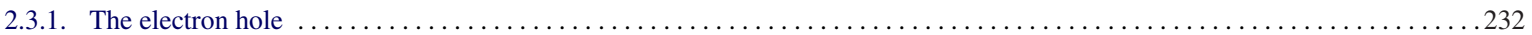

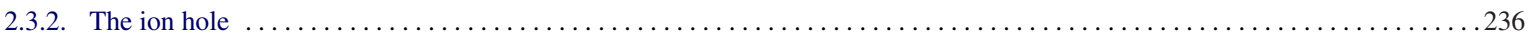

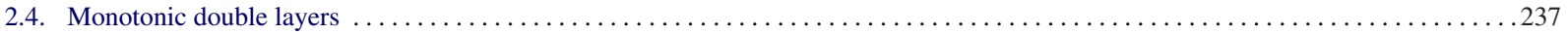

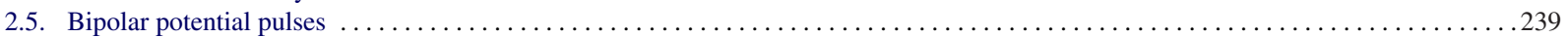

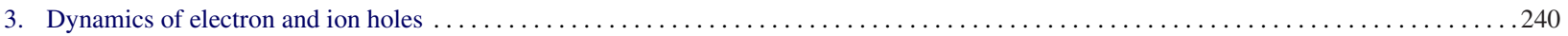

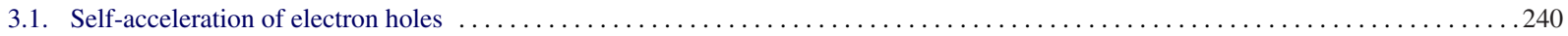

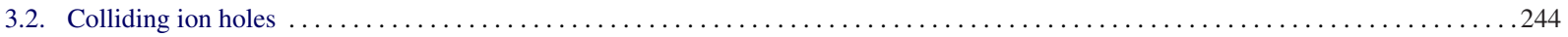

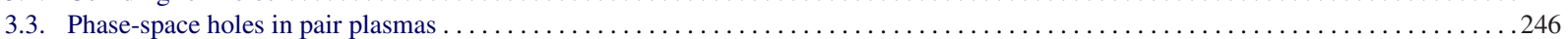

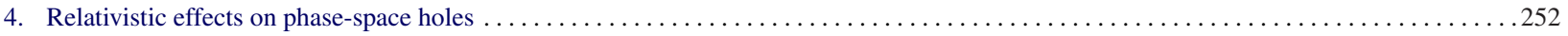

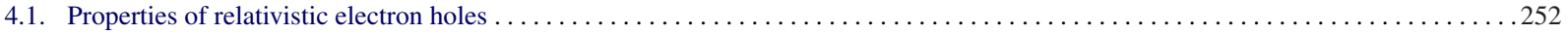

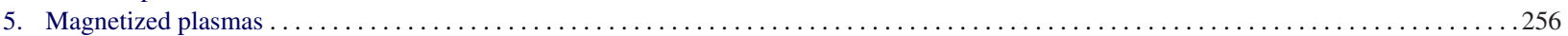

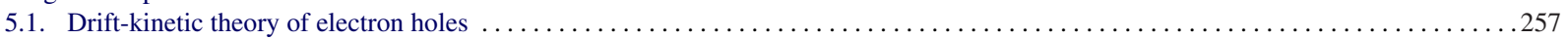

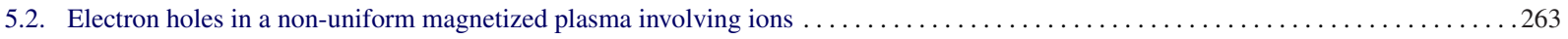

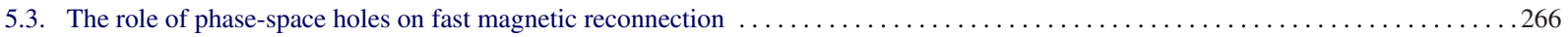

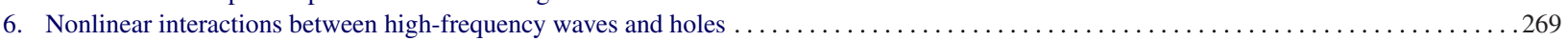

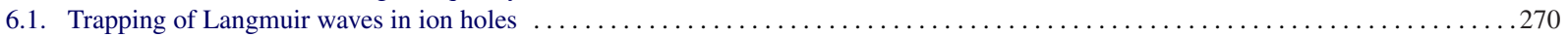

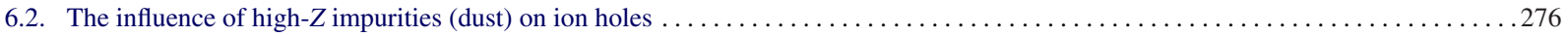

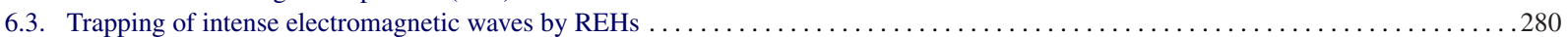

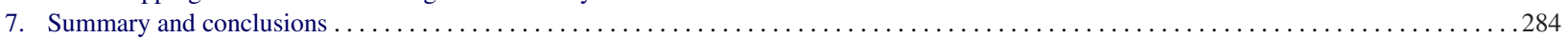

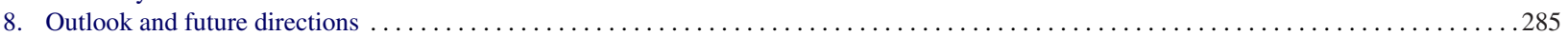

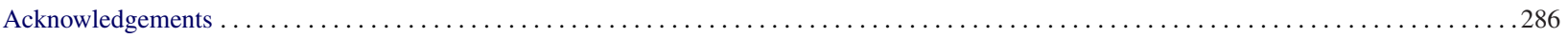

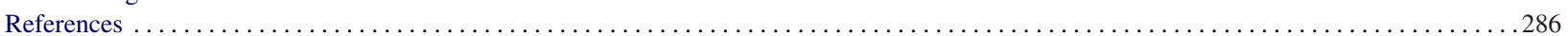

\section{Introduction}

Plasmas support a great variety of coherent nonlinear structures [1,2]. These include shocks, double layers, bare solitons (or solitary waves), envelope solitons, vortices, etc. Coherent nonlinear structures in plasmas involve dispersion and nonlinearities together with collisionless and collisional dissipation. Fluid and kinetic models are frequently used to investigate the formation and dynamics of numerous nonlinear structures which are observed in both laboratory and 
space plasmas. While in a fluid treatment one considers macroscopic aspects, a kinetic treatment provides the possibility of microscopic plasma behavior including the wave-particle interactions. Coherent nonlinear structures involving the latter are referred to as Bernstein-Greene-Kruskal (BGK) modes [3], which appear in plasmas with non-isothermal distribution functions and finite-amplitude electrostatic and electromagnetic fluctuations. The kinetic treatment extends also to the theory of incoherent light in nonlinear media which can be described with a Vlasov-like equation in some limits [4].

The electron and ion holes are BGK modes in which a population of charged particles (electrons, ions) is trapped in a self-created electrostatic potential which is positive for the electron hole and negative for the ion hole. Associated with the electron hole there is a depletion of the electrons at the center of the hole, while the ion hole is associated with a depletion of both the ion and electron number densities; the latter are due to the negative potential of the ion hole which repels the electrons locally. The existence of the ion hole is possible only if the electron temperature is sufficiently large so that the electrons can form a high-pressure background, which does not neutralize the ion density depletion of the ion hole. Generally speaking, the electron and ion holes are saturated states of two-stream unstable collisionless (or weakly collisional) plasmas in which the fluctuation growth is halted by the particle trapping in the wave potential. The trapping of plasma particles implies that the electron and ion holes are not amenable to macroscopic descriptions like the magnetohydrodynamic or other fluid descriptions. The Vlasov/Boltzmann picture has to be adopted for studying BGK modes in plasmas where the distribution functions of charged particles are non-Maxwellian.

The pioneering work of Bernstein, Greene and Kruskal led Dory [5] and Roberts and Berk [6] to carry out computer simulations of the dynamics of linearly unstable electron modes in one-dimensional (1D) electron plasma with a fixed ion background. They followed the motion of the phase-space boundaries of an incompressible and constant-density phase-space fluids, and observed the formation and condensation of electron holes, leading to long-lived nonlinear structures composed of depleted electron densities and rotating vortices in phase-space of trapped particles around the holes. Sakanaka [7] carried out numerical simulations of the ion-ion two-stream instability and found the appearance of a quiescent, heated dense region behind the shock, generated by the beam front in association with an ion phasespace vortex, which linked the hot state with the incoming two-stream state. Ion phase-space vortices thus play a role as fundamental as ion-acoustic shocks in plasmas. Numerical and theoretical studies of the interaction between the electron and ion holes have since then been performed by several authors. Newman et al. [8] studied numerically the dynamics and instability of 2D phase-space tubes, whereas Daldorff et al. [9] investigated the formation and dynamics of ion holes in three dimensions. Krasovsky et al. [10] showed theoretically and by computer simulations that the electron holes perform inelastic collisions. Vetoulis and Oppenheim [11] studied the radiation generation due to bounce resonances in electron holes. Guio et al. [12,13] investigated numerically the dynamics of phase-space vortices in a collisionless plasma, as well as the generation of phase-space structures by an obstacle in a streaming plasma. Saeki and Genma [14] examined the disruption of electron holes in an electron-ion plasma. The asymptotic nonlinear saturation of electrostatic waves has been treated both theoretically and numerically by several authors [15-17]. Dupree [18] found that the BGK mode is the most probable state for turbulent fluctuations which create self-trapping structures in a weakly turbulent plasma. It has also been pointed out that plasma waves can be undamped due to particle trapping effects in waves with arbitrarily small amplitudes [19,20]. Theoretical investigations of trapped particle effects in a magnetized plasma show that trapped ions influence strongly the electromagnetic ion-cyclotron waves [21].

About three decades ago, Schamel presented theories for the electron and ion holes [22-26], where a vortex distribution is assigned for the trapped particles and where the integration over the trapped and untrapped particle species in velocity space gives the particle number density as a function of the electrostatic potential. The latter is then calculated self-consistently from Poisson's equation. This model has been used in theoretical investigations for establishing existence criteria [26], and for determining the stability of the electron and ion holes [27,25,20], in addition to finding applications of solitary waves in coasting beams in synchrotrons [28]. Similar to the ion-acoustic waves in an electron-ion plasma [29], a modified Korteweg-de-Vries equation [30,31] can be derived for the dynamics of electron and ion holes in the small amplitude limit, but with a stronger nonlinearity due to the trapped particles [20].

The existence of the electron and ion holes has been demonstrated in both laboratory experiments and by satellites in the Earth's ionosphere and magnetosphere. In laboratory experiments, the formation and dynamics of solitary electron holes [32,33] and ion holes [34-37], as well as accelerated periodic ion holes [38] have been observed. Experiments with non-neutral plasmas in Penning-Malmberg traps have also shown trapped-particle modes associated with anomalous transport of the plasma particles across the external magnetic field [39]. Coherent nonlinear structures, often called electrostatic solitary waves (ESWs), are ubiquitous in the Earth's magnetosphere and in the interplanetary 
space. Numerous spacecraft missions have documented the characteristic signature of ESWs in the form of pulses of the electric field component parallel to the geomagnetic field direction. The spiky, bipolar and almost perfectly antisymmetric electric field signal parallel to the magnetic field, observed in satellite data, clearly results from the rapid propagation past the spacecraft of a structure which is localized along the magnetic field line and has a positive or negative potential. Most frequently these pulses are bipolar, i.e. they feature a positive and a negative signal (of the same intensity) of the electric field parallel to the magnetic field direction, along which they are propagating. They appear in the regions of near-Earth plasma that are prone to various linear instabilities due to the presence of either strong particle beams or sharp boundaries containing velocity shear, temperature, density and current gradients. The latter usually give rise to magnetic field explosions in association with non-isothermal particle distributions in space plasmas. The ion holes (or ion solitary waves) were detected first by the S3-3 [40] and Viking [41] spacecrafts, and recently the high-resolution measurements by the POLAR [42,43] and FAST (Fast Auroral SnapshoT) [44,45] satellites have provided data about their velocity and 3D spatial structures. They have been observed mostly in the low-altitude auroral zone, which is strongly magnetized and characterized by strong upward currents carried by ion beams travelling at $100-400 \mathrm{~km} / \mathrm{s}$. In some cases, they feature an overall potential drop, as those observed by Viking [41], and can be interpreted as weak double layers. Observations of broad electrostatic noise (BEN) by the GEOTAIL [46] and FAST spacecrafts [47-49] in the auroral acceleration regions have revealed that BEN is connected to solitary electron holes. Recent observations by the WIND satellite in the Earth's bow shock also reveal localized structures with bipolar electric fields, typical for the electron holes [50-52]. The geomagnetic field-aligned bipolar electric field pulses associated with electron phase-space holes have also been observed in the high-altitude acceleration region by the POLAR spacecraft [53] and in the magnetosheath and at the Earth's magnetopause by the POLAR and Cluster spacecrafts [43,54-56]. One-dimensional electron holes correlated with reconnection at the Earth's magnetotail have been recently observed by the Cluster spacecraft [57]. Electron solitary waves propagate along the geomagnetic field lines with a typical speed $250-2500 \mathrm{~km} / \mathrm{s}$, which is comparable to both the electron thermal speed and the speed of electron beams existing in those regions. Their amplitudes do not exceed the electron thermal energy, $e \phi<k_{\mathrm{B}} T_{e}$, while the duration is longer than the electron gyroperiod, sometimes as long as the gyroperiod of $\mathrm{H}^{+}$ions. They appear either isolated, or in a wavetrain organized with frequencies close to those of the ion-cyclotron or lower-hybrid waves [47]. The presence of the perpendicular, predominantly unipolar spikes of the electric field indicates a fully 3D geometry of the structure, but the actual shape is rather difficult to determine from the existing data. In the FAST data $[47,48]$, the parallel electric field is typically several times larger than the perpendicular one, and corresponds to the parallel size of $500 \mathrm{~m}$, i.e. longer than the local plasma Debye radius, $r_{\mathrm{D}} \sim 80 \mathrm{~m}$. However, the observed parallel and perpendicular electric fields in some cases were the same. Conversely, in the POLAR data [53,58] three somewhat different types of structures were observed, in which the perpendicular component of the electric field was either smaller or larger than the parallel electric field. In some events, it also possessed the same bipolar, rather than unipolar profile. These variations may be attributed to the different trajectories of the satellite through the structure. The presence of the perpendicular electric field leads to the transfer of the perpendicular momentum to the ions, which in the literature was referred to as 'effective perpendicular ion heating'. In this process, the perpendicular ion kinetic energy becomes comparable to the kinetic energy of the parallel electron streaming.

Nonlinear interactions between low-frequency ion-acoustic perturbations with high-frequency electromagnetic (EM) waves and Langmuir waves in a Maxwellian plasma have been considered by Hasegawa [59], Karpman [60-62] and Zakharov [63]. Here the high-frequency EM and Langmuir waves nonlinearly interact with low-frequency ion-acoustic perturbations via the ponderomotive force arising due to the spatial gradient of the high-frequency wave intensity. This nonlinear interaction is typically described by the hydrodynamic and Maxwell equations, and the governing equations admit the localization of EM and Langmuir wavepackets, leading to the formation of envelope EM and Langmuir wave solitons [64-67]. The latter are composed of electron/ion density depletion which traps EM and Langmuir wave envelops. A theory for Langmuir envelope solitons in an unmagnetized plasma with non-isothermal electron distribution function (trapped electrons in the ion wave potential) was developed by Schamel and Shukla [68]. Schamel and Maslov [69] studied theoretically the contraction of Langmuir waves trapped in a small-amplitude electron hole. Yan'kov [70] examined the response of kinetic untrapped ions in the Langmuir envelope solitary wave theory, and predicted the formation of sub ion thermal small-amplitude negative potential wells in an unmagnetized plasma. Mokhov and Chukbar [71] found a Langmuir envelope soliton accompanied with small-amplitude negative potential well created by localized Langmuir wave electric fields in a quasi-neutral plasma with non-isothermal ions whose temperature is much smaller than the electron temperature. Califano and Lontano [72] and Wang et al. [73] studied by Vlasov simulations 
the dynamics of finite-amplitude high-frequency Langmuir waves in 1D electron-ion plasma, where the wave collapse and heating of the electrons were observed. In two and three dimensions, one encounters EM wave self-focusing and Langmuir wave collapse [63,74,75]. The formation of cavitons has been observed in the Earth's ionosphere [76], as well as in several laboratory experiments [77-79].

Relativistic effects play a very important role in high-energy laser-plasma experiments [80,81], in plasma-based electron and photon accelerators [82-84], in supernova remnants and in gamma ray bursts [85], where electrons can be accelerated to relativistic energies by strong electrostatic fields. For short laser pulse intensities exceeding $10^{19} \mathrm{~W} / \mathrm{cm}^{2}$, the electrons in the laser beam oscillate relativistically. Interactions between intense short laser pulses and background plasma give rise to a number of nonlinear effects [80,82-84] associated with relativistic electron mass increase in the electromagnetic fields and the plasma density modification due to relativistic radiation ponderomotive force. In the past, several authors presented theoretical [86-88] and particle-in-cell (PIC) simulation studies [88-91] of intense electromagnetic envelope solitons in a cold plasma where the plasma slow response to the electromagnetic waves is modeled by the electron continuity and relativistic momentum equations, supplemented by Poisson's equation. Experimental observations [92] show bubble-like structures in proton images of laser-produced plasmas, which are interpreted as remnants of electromagnetic envelope solitons.

In this article, we present a comprehensive review of theoretical models and computer simulations of kinetic nonlinear structures involving phase-space vortices under various conditions. We start with the fundamental stationary theories for the electron and ion holes and double layers in the small and finite amplitude limits in an unmagnetized plasma, and then built up on recent developments in the area of coherent nonlinear structures via phase-space vortices. We show that one can have both monopolar potential pulses as well as double layers characterized by a potential step in electron-ion plasmas with non-isothermal electron and ion distribution functions. We present recent studies of the hole dynamics in an electron-ion plasma, in addition to the formation and dynamics of large amplitudes holes in a pair-ion plasma. Our simulation results of the complex dynamics of electron and ion holes interacting with the background plasma exhibit that the dynamics of the electron holes is significantly affected by the ion dynamics. Specifically, Vlasov simulations of fully nonlinear interactions between the electron holes and oxygen ions show that large-amplitude electron hole potential accelerates the ions locally and that the self-created ion density cavity, in turn, accelerates the electron hole which propagates away from the ion cavity with a constant speed close to half the electron thermal speed. Furthermore, colliding ion holes may lead to non-Maxwellian electron distributions due to the acceleration of electrons in the negative ion hole potential, which works as a barrier for the electrons. Streams of electrons are accelerated by colliding ion holes, and these electron streams excite high-frequency Langmuir waves. The electron hole theory has been further extended to include relativistic electron mass increase in a relativistically hot plasma. Here the distribution functions for untrapped and trapped electrons are solutions of the relativistic Vlasov equation in which the unperturbed distribution function is of a relativistic Jüttner-Synge form. Poisson's equation with the relativistic distribution function then determines the existence and profile of the localized REHs. The amplitudes and widths of the latter are much larger than those of non-relativistic electron holes in an unmagnetized plasma. The influence of an external magnetic field and the plasma inhomogeneity on the formation of electron and ion holes is also examined. We find that the external magnetic field produces anisotropic holes that have differential scale sizes along and across the magnetic field direction. The effect of the plasma inhomogeneity is to introduce a new class of bipolar pulses that move with the electron diamagnetic speed, and which have a stronger electric field strength on account of the trapped electron-induced nonlinearity in our non-uniform plasma. In plasmas, we can have the simultaneous presence of high-frequency plasma waves and kinetic structures. In such a situation, intense high-frequency fields can nonlinearly interact with electron and ion holes. We find that Langmuir waves can be trapped in the density cavity of an ion hole. Numerical studies show that trapped Langmuir waves are Landau damped due to the relatively small size of the ion holes. The effect of high- $Z$ impurities on the trapping of Langmuir waves in ion holes is also examined. Finally, we present an interesting investigation of intense electromagnetic wave localization and its trapping in REHs in a relativistically hot plasma.

The manuscript is organized in the following fashion. In Section 2, we review the general theory of electron and ion holes and monotonic double layers based on Schamel's solution of the quasi-stationary Vlasov-Poisson system. The dynamics of the electron and ion holes and their interactions with the plasma and kinetic electrons are discussed in Section 3. An extension of Schamel's theory for the electron holes to account for relativistic effects is presented in Section 4, while extensions of the theory for the electron holes to a uniform and non-uniform magnetoplasmas is presented in Section 5. In Section 6, we study nonlinear interactions between high-frequency waves and phase-space holes and demonstrate the trapping of Langmuir waves in ion holes and the trapping of electromagnetic waves in REHs. 
The main results of the present article are summarized in Section 7. Possible extensions of the presented theoretical and numerical results is discussed in Section 8.

\section{General theory of electron and ion holes}

\subsection{Governing equations}

The dynamics of electrons and ions in an unmagnetized, collisionless plasma is governed by the Vlasov-Poisson system of equations

$$
\frac{\partial f_{j}}{\partial t}+v \frac{\partial f_{j}}{\partial x}-\frac{q_{j}}{m_{j}} \frac{\partial \varphi}{\partial x} \frac{\partial f_{j}}{\partial v}=0
$$

and

$$
\frac{\partial^{2} \varphi}{\partial x^{2}}=\frac{e}{\epsilon_{0}}\left(n_{\mathrm{e}}-n_{\mathrm{i}}\right),
$$

where the particle number density is

$$
n_{j}=\int_{-\infty}^{\infty} f_{j} \mathrm{~d} v
$$

where $f_{j}$ is the distribution function of particle species $j$ ( $j$ equals e for electrons and i for ions), $m_{j}$ is the mass, $q_{\mathrm{e}}=-e$, $q_{\mathrm{i}}=e, e$ is the magnitude of the electron charge, and $\varphi$ is the electrostatic potential.

\subsection{Excitation of phase-space holes}

The generation of phase-space holes is a natural nonlinear saturation mechanism for large-amplitude electrostatic waves resulting from streaming instabilities or from other free energy sources in plasmas. The creation of periodic non-relativistic electron holes by a large-amplitude electrostatic wave is illustrated in Fig. 1, where we have solved numerically the Vlasov equation for the electrons with a neutralizing ion background of constant particle density, $n_{\mathrm{i}}=n_{0}$ [109]. The electrons are assumed to form a large-amplitude, density-modulated wave with the electron distribution function of the form $f_{\mathrm{e}}=\left[n_{0} /(2 \pi)^{1 / 2} V_{T e}\right]\left[1+0.5 \cos \left(0.5 x / r_{\mathrm{De}}\right)\right] \exp \left(-v^{2} / 2 V_{T e}^{2}\right)$ (depicted in the left panel of Fig. 1), which is released at $t=0 \omega_{\mathrm{pe}}^{-1}$, and is allowed to evolve in time. Here $V_{T e}=\left(k_{\mathrm{B}} T_{\mathrm{e}} / m_{\mathrm{e}}\right)^{1 / 2}$ is the electron thermal speed, $k_{\mathrm{B}}$ is Boltzmann's constant, $T_{\mathrm{e}}$ is the electron temperature, $r_{\mathrm{De}}=\left(\epsilon_{0} k_{\mathrm{B}} T_{\mathrm{e}} / n_{0} e^{2}\right)^{1 / 2}$ is the electron Debye radius, $n_{0}$ is the equilibrium electron number density, $\epsilon_{0}$ is the electric permittivity in vacuum, and $\omega_{\mathrm{pe}}=\left(n_{0} e^{2} / \epsilon_{0} m_{\mathrm{e}}\right)^{1 / 2}$ is the electron plasma frequency. Fig. 1 exhibits that at $t=7 \omega_{\mathrm{pe}}^{-1}$ some of the electrons have been accelerated by the self-consistent electrostatic field and have formed streams in velocity space. At $t=70 \omega_{\mathrm{pe}}^{-1}$ the streams of electrons have mixed in a complex fashion. Two electron holes have been created at $v \approx \pm 3 V_{T e}$, associated with a depletion of the electron distribution function and a population of trapped electrons moving with the electron holes.

In Fig. 2, we show the electron phase-space distribution from a PIC simulation [93] of a mildly relativistic ion beam instability. Here, the ion beam is moving with the speed $0.8 c$ ( $c$ is the speed of light in vacuum) relative to the bulk plasma, and excites large-amplitude electrostatic Langmuir waves with approximately the same phase speed as the ion beam. The instability saturates by the trapping of electrons and the formation of the periodic electron holes displayed in Fig. 2. It is found that relativistic effects play an important role for the stabilization of the periodic BGK modes against the sideband (coalescence) instability [94,95], in which they tend to merge and collapse [96].

\subsection{Theory of quasi-stationary phase-space holes}

In order to describe the electron and ion holes mathematically, we look for quasi-stationary solutions moving with constant speeds $u_{j 0}$. In this case, we introduce the ansatz $f_{j}=f_{j}(\xi, v)$ and $\varphi=\varphi(\xi)$, where $\xi=x-u_{j 0} t$, which 

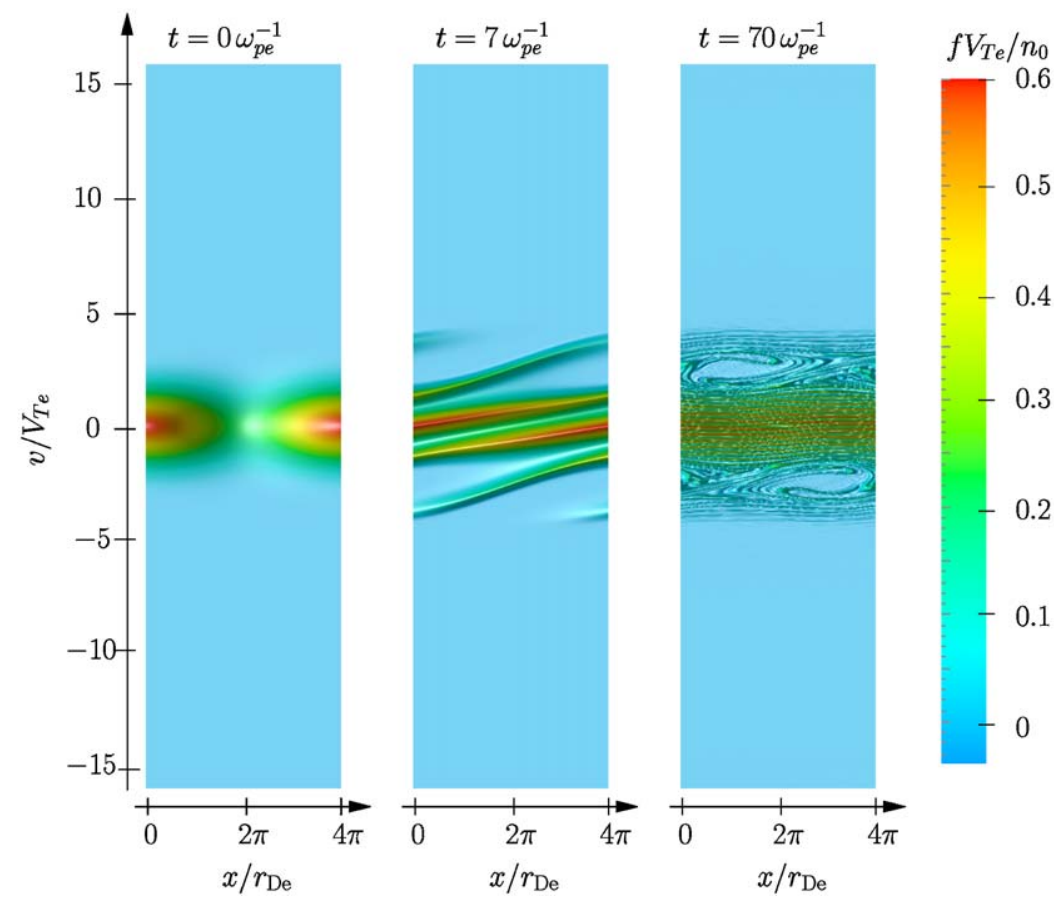

Fig. 1. The electron distribution function at $t=0 \omega_{\mathrm{pe}}^{-1}$ (left panel), $t=7 \omega_{\mathrm{pe}}^{-1}$ (middle panel) and $t=70 \omega_{\mathrm{pe}}^{-1}$ (lower panel). (After Ref. [109].)

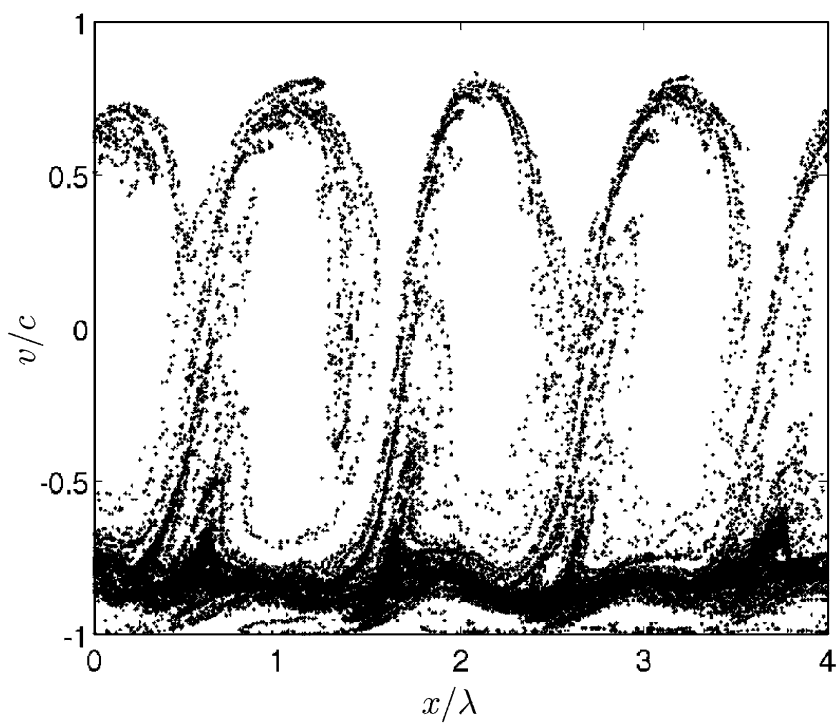

Fig. 2. The electron distribution function taken from a PIC simulation of a mildly relativistic ion beam instability, in a frame moving with the ion beam. Here, $\lambda$ denotes the principal wavelength of the excited wave. (After Ref. [93].)

transforms the system of Eqs. (1) and (2) to a new set of equations

$$
\left(v-u_{j 0}\right) \frac{\partial f_{j}}{\partial \xi}-\frac{q_{j}}{m_{j}} \frac{\partial \varphi}{\partial x} \frac{\partial f_{j}}{\partial v}=0
$$




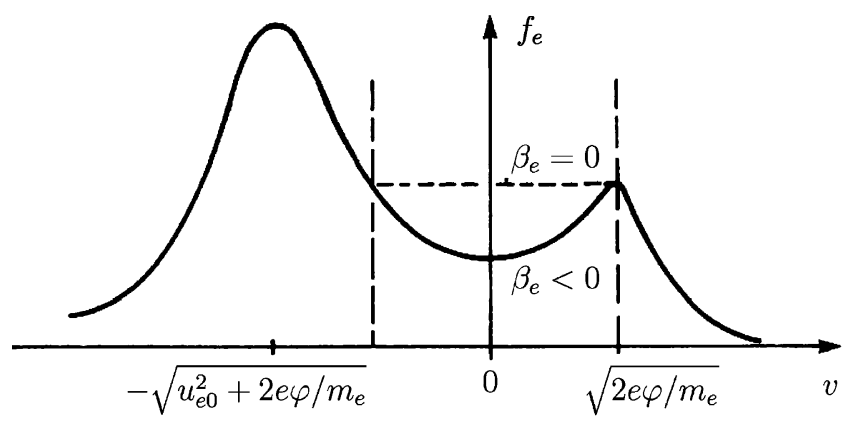

Fig. 3. The electron distribution function in velocity space. In a frame moving with speed of the electron hole, the electrons with a velocity $|v|<\sqrt{2 e \phi / m_{\mathrm{e}}}$ are trapped. Negative $\beta_{\mathrm{e}}$ corresponds to an excavated vortex distribution, while $\beta_{\mathrm{e}}=0$ corresponds to a flat (constant) distribution. (After Ref. [25].)

and

$$
\frac{\mathrm{d}^{2} \varphi}{\mathrm{d} \xi^{2}}=\frac{e}{\epsilon_{0}}\left(n_{\mathrm{e}}-n_{\mathrm{i}}\right),
$$

where $n_{\mathrm{e}}$ and $n_{\mathrm{i}}$ are given by Eq. (3). Eq. (4) can be integrated along its particle trajectories, so that $f_{j}$ is a function of the particle energies

$$
\mathscr{E}_{\mathrm{e}}=\frac{m_{\mathrm{e}}\left(v-u_{\mathrm{e} 0}\right)^{2}}{2}-e \varphi
$$

and

$$
\mathscr{E}_{\mathrm{i}}=\frac{m_{\mathrm{i}}\left(v-u_{\mathrm{i} 0}\right)^{2}}{2}+e \varphi
$$

for the electrons and ions, respectively. Here $\mathscr{E}_{j} \geqslant 0$ corresponds to untrapped (free) particles, while $\mathscr{E}_{j}<0$ corresponds to trapped particles.

\subsubsection{The electron hole}

An electron hole is characterized by a localized positive potential, in which a population of negatively charged electrons can be trapped. A special class of electron hole solutions can be found by prescribing the distribution function for the free and trapped electrons [22,23], viz.

$$
f_{\mathrm{e}}= \begin{cases}\frac{n_{0}}{(2 \pi)^{1 / 2} V_{T e}} \exp \left\{-\frac{1}{k_{\mathrm{B}} T_{\mathrm{e}}}\left[\sigma_{\mathrm{e}} \mathscr{E}_{\mathrm{e}}^{1 / 2}+\left(\frac{m_{\mathrm{e}} u_{\mathrm{e} 0}^{2}}{2}\right)^{1 / 2}\right]^{2}\right\}, & \mathscr{E}_{\mathrm{e}}>0, \\ \frac{n_{0}}{(2 \pi)^{1 / 2} V_{T e}} \exp \left[-\frac{1}{k_{\mathrm{B}} T_{\mathrm{e}}}\left(\beta_{\mathrm{e}} \mathscr{E}_{\mathrm{e}}+\frac{m_{\mathrm{e}} u_{\mathrm{e} 0}^{2}}{2}\right)\right], & \mathscr{E}_{\mathrm{e}}<0,\end{cases}
$$

where $\sigma_{\mathrm{e}}=\operatorname{sign}\left(v-u_{\mathrm{e} 0}\right)$ and $\beta_{\mathrm{e}}$ is the trapping parameter describing the "temperature" of the trapped electrons. Negative values of $\beta_{\mathrm{e}}$, which we are interested in here, lead to a vortex distribution for the trapped electrons, where the distribution is excavated in velocity space; see Fig. 3, while $\beta_{\mathrm{e}}=0$ leads to a flat distribution in velocity space. Electrons with negative energies are trapped locally in the electron distribution function as illustrated in Fig. 4, where they propagate along closed trajectories in phase space, while electrons with positive energy propagate along open trajectories, in a frame moving with the electron hole. In the study of electron holes that are moving much faster than the ion sound speed, one can assume that the ions form a constant neutralizing background, and write

$$
n_{\mathrm{i}}=n_{0} .
$$




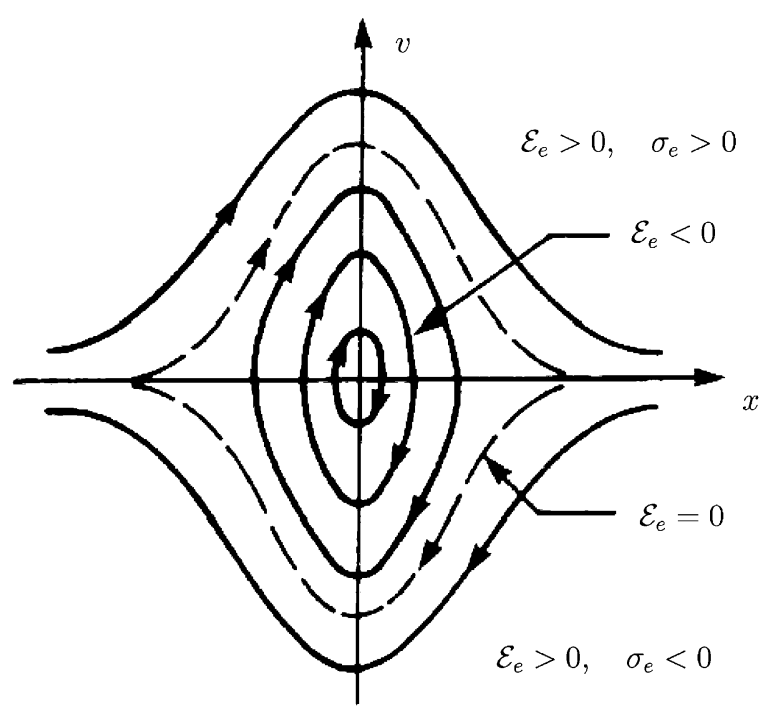

Fig. 4. Particle trajectories in the vicinity of an electron hole, for different energies $\mathscr{E}$ e. Trapped electrons have negative energies $(\mathscr{E}$ e $<0)$, while free electrons have positive energies $(\mathscr{E} \mathrm{e}>0)$. Here, $\sigma_{\mathrm{e}}$ denotes the sign of the velocities of the free electrons, in a frame moving with the speed of the electron hole (after Ref. [25]).

This approximation clearly breaks down if the electron holes have a very low speed so that the background ions have the time to be accelerated by the positive electron hole potential, or for a plasma with equal or comparable masses of the positive and negative particle species. These cases will be discussed in Sections 3.1 and 3.3, respectively.

An integration of the electron distribution function over velocity space gives the electron number density as a function of the electrostatic potential [26]. We have

$$
n_{\mathrm{e}}=n_{0} \exp \left(-\frac{M_{\mathrm{e}}^{2}}{2}\right)\left[I\left(\phi_{\mathrm{e}}\right)+\kappa\left(\frac{M_{\mathrm{e}}^{2}}{2}, \phi_{\mathrm{e}}\right)+\frac{2 W_{\mathrm{D}}\left[\left(-\beta_{\mathrm{e}} \phi_{\mathrm{e}}\right)^{1 / 2}\right]}{\left(\pi\left|\beta_{\mathrm{e}}\right|\right)^{1 / 2}}\right],
$$

where we have denoted the electron Mach number $M_{\mathrm{e}}=u_{\mathrm{e} 0} / V_{T e}$ and the normalized potential $\phi_{\mathrm{e}}=e \varphi / k_{\mathrm{B}} T_{\mathrm{e}}$. The special functions are defined as [22,26]

$$
\begin{aligned}
& I(\Phi)=\exp (\Phi)[1-\operatorname{erf}(\sqrt{\Phi})] \\
& \kappa(X, \Phi)=\frac{2}{\sqrt{\pi}} \int_{0}^{\pi / 2} \sqrt{X} \cos \theta \exp \left(-\Phi \tan ^{2} \theta+X \cos ^{2} \theta\right) \operatorname{erf}(\sqrt{X} \cos \theta) \mathrm{d} \theta
\end{aligned}
$$

and the Dawson integral is

$$
W_{\mathrm{D}}(u)=\exp \left(-u^{2}\right) \int_{0}^{u} \exp \left(t^{2}\right) \mathrm{d} t .
$$

The ion and electron number densities inserted into Eq. (5) give the equation for the self-consistent potential of the electron hole.

In order to gain insight into the electron hole, we consider the small-amplitude limit (viz. $\left.\phi_{\mathrm{e}} \ll 1\right)$. To leading order, we then have $[24,20]$

$$
n_{\mathrm{e}}=n_{0}\left(1+a_{\mathrm{e}} \phi_{\mathrm{e}}-\frac{4}{3} b_{\mathrm{e}} \phi_{\mathrm{e}}^{3 / 2}\right)
$$

where

$$
a_{\mathrm{e}}=-\frac{1}{2} Z_{r}^{\prime}\left(\frac{M_{\mathrm{e}}}{\sqrt{2}}\right)
$$




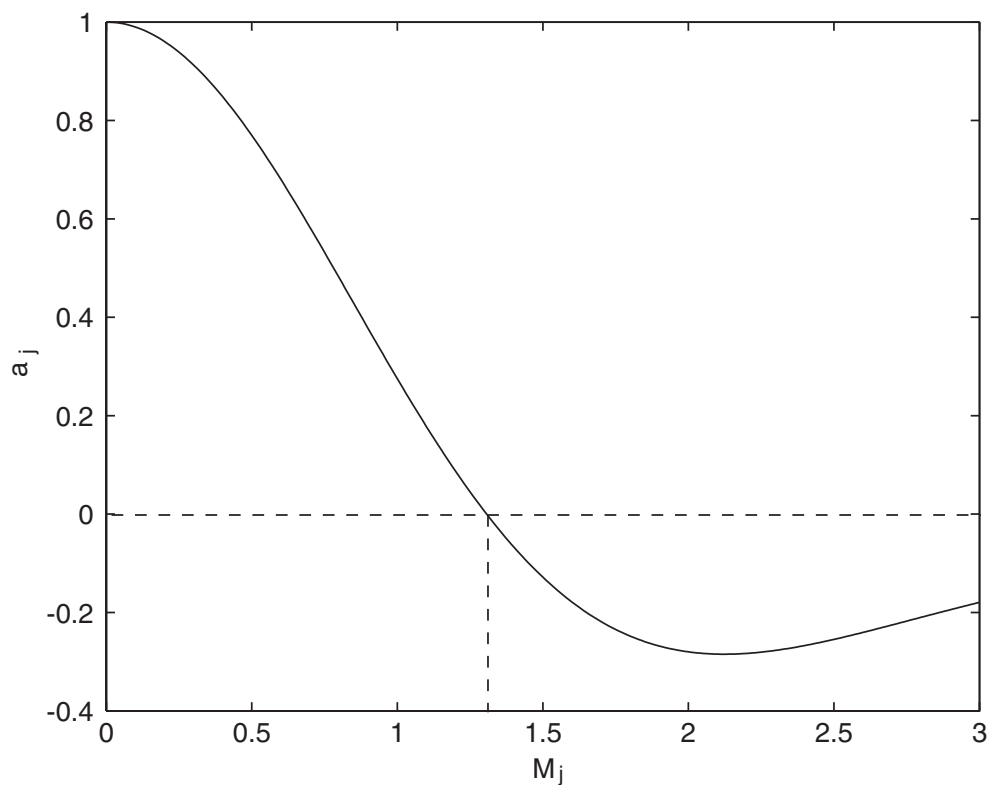

Fig. 5. The function $a_{j}=-(1 / 2) Z_{r}^{\prime}\left[M_{j} / \sqrt{2}\right]$ as a function of $M_{j}$, where $j$ equals e for electrons and i for ions.

and the real part of the plasma dispersion function $Z(u)$ is

$$
Z_{r}(u)=\pi^{-1 / 2} p \int_{-\infty}^{\infty} \frac{\exp \left(-t^{2}\right)}{t-u} \mathrm{~d} t=-2 W_{\mathrm{D}}(u)
$$

and its derivative

$$
Z_{r}^{\prime}(u)=-2 \pi^{-1 / 2} p \int_{-\infty}^{\infty} \frac{t \exp \left(-t^{2}\right)}{t-u} \mathrm{~d} t=-2\left[1-2 u W_{\mathrm{D}}(u)\right],
$$

where $p$ denotes the principal value of the integral. In Fig. 5 we have plotted the function $a_{j}$ as a function of $M_{j}$ (where $j$ equals $e$ for electrons and $i$ for ions). The factor in front of the nonlinearity in Eq. (14) is

$$
b_{\mathrm{e}}=\pi^{-1 / 2} \exp \left(-M_{\mathrm{e}}^{2} / 2\right)\left(1-\beta_{\mathrm{e}}-M_{\mathrm{e}}^{2}\right) .
$$

Poisson's equation (5) with the ion and electron number densities given by Eqs. (9) and (14) can be written in the form of an energy integral

$$
\frac{r_{\text {De }}^{2}}{2}\left[\phi_{\mathrm{e}}^{\prime}(\xi)\right]^{2}+V\left(\phi_{\mathrm{e}} ; M_{\mathrm{e}}, \beta_{\mathrm{e}}\right)=0
$$

where the Sagdeev potential is

$$
V\left(\phi_{\mathrm{e}} ; M_{\mathrm{e}}, \beta_{\mathrm{e}}\right)=-\frac{a_{\mathrm{e}}}{2} \phi_{\mathrm{e}}^{2}+\frac{8}{15} b_{\mathrm{e}} \phi_{\mathrm{e}}^{5 / 2} .
$$

For a bell-shaped potential having a maximum value $\phi_{\mathrm{e}}=\psi_{\mathrm{e}}$ at $\xi=0$, we have $V\left(\psi_{\mathrm{e}} ; M_{\mathrm{e}}, \beta_{\mathrm{e}}\right)=0$ at $\xi=0$ and at $|\xi| \rightarrow \infty$, and $V\left(\phi_{\mathrm{e}} ; M_{\mathrm{e}}, \beta_{\mathrm{e}}\right)<0$ in $0<\phi_{\mathrm{e}}<\psi_{\mathrm{e}}$. We thus have the nonlinear dispersion relation

$$
a_{\mathrm{e}}-\frac{16}{15} b_{\mathrm{e}} \psi_{\mathrm{e}}^{1 / 2}=0,
$$

which can be solved for the electron hole speed, yielding

$$
u_{\mathrm{e} 0}=1.305 V_{T e}\left(1-\frac{16}{15} b_{\mathrm{e}} \psi_{\mathrm{e}}^{1 / 2}\right) .
$$



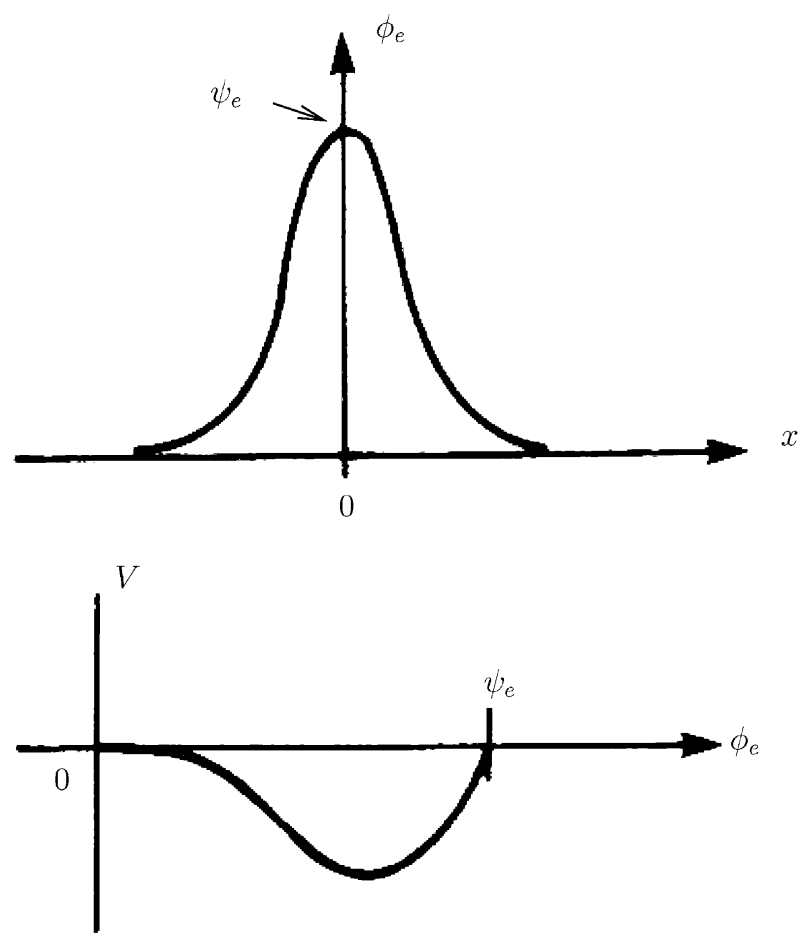

Fig. 6. The electrostatic potential and classical potential for a stationary electron hole. (After Ref. [25].)

This shows that the electron hole is a nonlinear descendent of a non-resonant electron thermal mode. The associated Sagdeev potential and the electron hole potential distribution are, respectively,

$$
V\left(\phi_{\mathrm{e}} ; M_{\mathrm{e}}, \beta_{\mathrm{e}}\right)=-\frac{8}{15} b_{\mathrm{e}} \phi_{\mathrm{e}}^{2}\left(\sqrt{\psi_{\mathrm{e}}}-\sqrt{\phi_{\mathrm{e}}}\right)
$$

and

$$
\phi_{\mathrm{e}}(\xi)=\psi_{\mathrm{e}} \operatorname{sech}^{4}\left(\xi / l_{\mathrm{e}}\right)
$$

where the electron hole width is $l_{\mathrm{e}}=\sqrt{15 / b_{\mathrm{e}} \psi_{\mathrm{e}}^{1 / 2}}$. The typical profile of the electrostatic potential is shown in Fig. 6 . In order for the Sagdeev potential to be negative in the region $0<\phi_{\mathrm{e}}<\psi_{\mathrm{e}}$, we should have both $a_{\mathrm{e}}>0$ and $b_{\mathrm{e}}>0$. The latter condition is violated for $M_{\mathrm{e}}$ larger than approximately 1.305 when $a_{\mathrm{e}}$ becomes negative; see Fig. 5. Thus, there does not exist solitary electron holes with speeds $M_{\mathrm{e}}>1.305$. When $M_{\mathrm{e}} \lesssim 1.305$, we see from Eq. (18) that we have the condition $\beta_{\mathrm{e}}<1-M_{\mathrm{e}}^{2} \approx 0.71$ for $b_{\mathrm{e}}$ to be positive, while the restriction is released for smaller $M_{\mathrm{e}}$.

The potential form of the electron hole deviates from that of the Korteweg-de Vries (K-dV) soliton associated with the nonlinear ion-acoustic wave, which is given by

$$
\phi_{\mathrm{e}}(\xi)=\psi_{\mathrm{e}} \operatorname{sech}^{2}\left(\sqrt{\psi_{\mathrm{e}} / 6} \xi\right) .
$$

We see that the electron hole is narrower than the $\mathrm{K}-\mathrm{dV}$ soliton for small amplitudes. The electron density is diminished at the center, viz

$$
n_{\mathrm{e}} \approx 1-\frac{4}{15} \psi_{\mathrm{e}}^{3 / 2}
$$

The evolution equation for the electron hole is the modified $\mathrm{K}-\mathrm{dV}$ (MK-dV) equation [25]

$$
\frac{\partial \phi_{\mathrm{e}}}{\partial t}+1.305\left(1-2 b_{\mathrm{e}} \sqrt{\phi_{\mathrm{e}}}\right) \frac{\partial \phi_{\mathrm{e}}}{\partial x}-1.305 \frac{\partial^{3} \phi_{\mathrm{e}}}{\partial x^{3}}=0
$$


with a square-root nonlinearity. The MK-dV equation possesses a finite set of conservation laws only and does not belong to the class of integrable differential equations.

\subsubsection{The ion hole}

An ion hole is characterized by a localized negative potential, which can trap a population of the ions. Similarly as for the electron holes, a special class of solutions can be found by prescribing the ion distribution function for free $\left(\mathscr{E}_{\mathrm{i}}>0\right)$ and trapped $\left(\mathscr{E}_{\mathrm{i}}<0\right)$ ions as [26]

$$
f_{\mathrm{i}}= \begin{cases}\frac{n_{0}}{(2 \pi)^{1 / 2} V_{T i}} \exp \left\{-\frac{1}{k_{\mathrm{B}} T_{\mathrm{i}}}\left[\sigma_{\mathrm{i}} \mathscr{E}_{\mathrm{i}}^{1 / 2}+\left(\frac{m_{\mathrm{i}} u_{\mathrm{i} 0}^{2}}{2}\right)^{1 / 2}\right]^{2}\right\}, & \mathscr{E}_{\mathrm{i}}>0, \\ \frac{n_{0}}{(2 \pi)^{1 / 2} V_{T i}} \exp \left[-\frac{1}{k_{\mathrm{B}} T_{\mathrm{i}}}\left(\beta_{\mathrm{i}} \mathscr{E}_{\mathrm{i}}+\frac{m_{\mathrm{i}} u_{\mathrm{i} 0}^{2}}{2}\right)\right], & \mathscr{E}_{\mathrm{i}}<0,\end{cases}
$$

where $V_{T i}=\left(k_{\mathrm{B}} T_{\mathrm{i}} / m_{\mathrm{i}}\right)^{1 / 2}$ is the ion thermal speed, $T_{\mathrm{i}}$ is the ion temperature, $\sigma_{\mathrm{i}}=\operatorname{sign}\left(v-u_{\mathrm{i} 0}\right)$ and $\beta_{\mathrm{i}}$ is the ion trapping parameter, which describes the "temperature" of the trapped ions. We are interested in negative values of $\beta_{\mathrm{i}}$, giving an excavated vortex distribution of the ions. In the study of ion holes, the electrons are usually assumed to be Maxwell-Boltzmann distributed so that

$$
f_{\mathrm{e}}=\frac{n_{0}}{(2 \pi)^{1 / 2} V_{T e}} \exp \left(\frac{e \varphi}{k_{\mathrm{B}} T_{\mathrm{e}}}-\frac{v^{2}}{2 V_{T_{\mathrm{e}}}^{2}}\right)
$$

which simplifies the mathematics somewhat compared to using an exact solution of the Vlasov equation for the electrons. Integrating the ion and electron distribution functions over velocity space, we obtain the particle densities

$$
n_{\mathrm{i}}=n_{0} \exp \left(-\frac{M_{\mathrm{i}}^{2}}{2}\right)\left\{I\left(-\phi_{\mathrm{i}}\right)+\kappa\left(\frac{M_{\mathrm{i}}^{2}}{2},-\phi_{\mathrm{i}}\right)+\frac{2}{\sqrt{\pi\left|\beta_{\mathrm{i}}\right|}} W_{\mathrm{D}}\left[\sqrt{\beta_{\mathrm{i}} \phi_{\mathrm{i}}}\right]\right\}
$$

and

$$
n_{\mathrm{e}}=n_{0} \exp \left(\tau \phi_{\mathrm{i}}\right)
$$

where we have denoted the ion Mach number $M_{\mathrm{i}}=u_{\mathrm{i} 0} / V_{T i}$, the normalized potential $\phi_{\mathrm{i}}=e \varphi / k_{\mathrm{B}} T_{\mathrm{i}}$, and $\tau=T_{\mathrm{i}} / T_{\mathrm{e}}$. The ion and electron densities inserted into Eq. (5) gives the equation for the self-consistent potential of the ion hole.

In the small-amplitude limit (viz. $\phi_{\mathrm{i}} \ll 1$ ), we have to leading order [20]

$$
n_{\mathrm{i}}=n_{0}\left[1-a_{\mathrm{i}} \phi_{\mathrm{i}}-\frac{4}{3} b_{\mathrm{i}}\left(-\phi_{\mathrm{i}}\right)^{3 / 2}\right] \text {, }
$$

where

$$
a_{\mathrm{i}}=-\frac{1}{2} Z_{r}^{\prime}\left(\frac{M_{\mathrm{i}}}{\sqrt{2}}\right)
$$

which is plotted in Fig. 5, and

$$
b_{\mathrm{i}}=\pi^{-1 / 2}\left(1-\beta_{\mathrm{i}}-M_{\mathrm{i}}^{2}\right) \text {. }
$$

The electron density is

$$
n_{\mathrm{e}}=n_{0}\left(1+\tau \phi_{\mathrm{i}}\right) .
$$

Poisson's equation (5) with the ion and electron densities given by Eqs. (32) and (35), respectively, can be written in the form of an energy integral

$$
\frac{\tau r_{\text {De }}^{2}}{2}\left[\phi_{\mathrm{i}}^{\prime}(\xi)\right]+V\left(\phi_{\mathrm{i}} ; M_{\mathrm{i}}, \beta_{\mathrm{i}}, \tau\right)=0,
$$




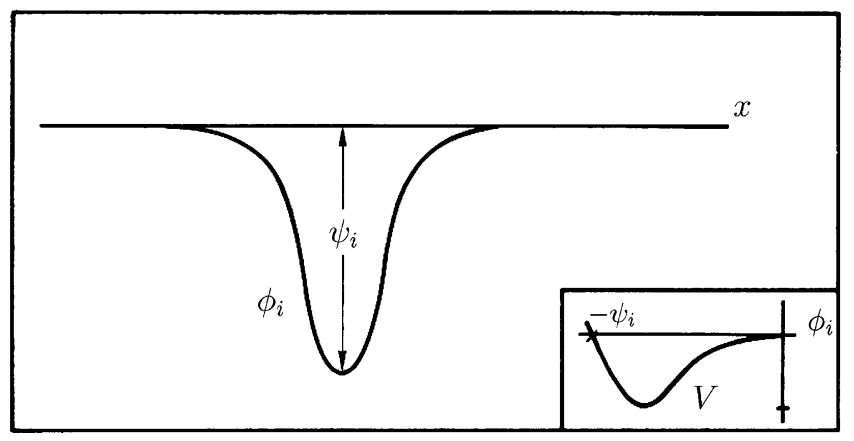

Fig. 7. The electrostatic potential and classical potential for a stationary ion hole. (After Ref. [25].)

where the Sagdeev potential associated with the ion hole is

$$
V\left(\phi_{\mathrm{i}} ; M_{\mathrm{i}}, \beta_{\mathrm{i}}, \tau\right)=\frac{1}{\tau}\left[-\frac{\left(a_{\mathrm{i}}+\tau\right)}{2} \phi_{\mathrm{i}}^{2}+\frac{8}{15} b_{\mathrm{i}}\left(-\phi_{\mathrm{i}}\right)^{5 / 2}\right]
$$

The electric potential has a minimum $\phi_{\mathrm{i}}=-\psi_{\mathrm{i}}$ where $\partial \phi_{\mathrm{i}} / \partial \xi=0$. From the energy law it then follows that $V\left(-\psi_{\mathrm{i}}\right)=0$ or

$$
\left(\tau+a_{\mathrm{i}}\right)=\frac{16}{15} b_{\mathrm{i}} \sqrt{\psi_{\mathrm{i}}},
$$

which relates the Mach number $M_{\mathrm{i}}$, hidden in $a_{\mathrm{i}}$ and $b_{\mathrm{i}}$, and the amplitude $\psi_{\mathrm{i}}$, and can therefore be regarded as a nonlinear dispersion relation. Since $V$ must be negative between $\phi_{\mathrm{i}}=0$ and $\phi_{\mathrm{i}}=-\psi$, well-defined ion holes exist only if $b_{\mathrm{i}}>0$, which together with Eq. (34) implies $\beta_{\mathrm{i}}<1-M_{\mathrm{i}}^{2}$ for the existence of the ion hole. We see in Fig. 5 that $a_{\mathrm{i}}$ becomes negative at $M_{\mathrm{i}} \approx 1.305$, which from Eq. (38) implies that $\tau$ must be sufficiently large if $M_{\mathrm{i}}>1.305$, for the existence of the ion hole. The associated Sagdeev potential and the potential profile of the ion hole are, respectively,

$$
V\left(\phi_{\mathrm{i}}\right)=-\frac{8 b_{\mathrm{i}}}{15 \tau} \phi_{\mathrm{i}}^{2}\left(\sqrt{\psi_{\mathrm{i}}}-\sqrt{-\phi_{\mathrm{i}}}\right)
$$

and

$$
\phi_{\mathrm{i}}(\xi)=-\psi_{\mathrm{i}} \operatorname{sech}^{4}\left(\xi / l_{\mathrm{i}}\right)
$$

where the width of the ion hole is $l_{\mathrm{i}}=\sqrt{15 / b_{\mathrm{i}} \psi_{\mathrm{i}}^{1 / 2}}$. The depleted ion density at the center of the ion hole is $n_{\mathrm{i}}=1-a_{\mathrm{i}} \psi_{\mathrm{i}}-(4 / 15) b_{\mathrm{i}} \psi_{\mathrm{i}}^{3 / 2}$. The depth is stronger the higher the ion temperature. Since the electron density also forms a cavity due to the negative potential, the whole plasma density is depleted locally in the ion hole. A typical profile of the ion hole electrostatic potential is displayed in Fig. 7.

The evolution equation which governs the propagation of the ion hole reads [97]

$$
\frac{\partial \phi_{\mathrm{i}}}{\partial t}+1.305\left(1+\tau-2 b_{\mathrm{i}}\left|\phi_{\mathrm{i}}\right|^{1 / 2}\right) \frac{\partial \phi_{\mathrm{i}}}{\partial x}-1.305 \frac{\partial^{3} \phi_{\mathrm{i}}}{\partial x^{3}}=0
$$

\subsection{Monotonic double layers}

A double layer (DL) is defined as a monotonic transition of the electric potential connecting smoothly two differently biased plasmas [98]. This is achieved by a dipole-like charge distribution, as shown in Fig. 8, where the electric and classical potentials are displayed together with the total charge density $\rho$. Poisson's equation dictates that a positively charged layer gives rise to a region of a negative curvature of $\phi$ and vice versa. Hence, two oppositely charged layers are needed to built up the DL structure. Double layers solutions must satisfy $V(0)=V(\psi)=0$ and $(\mathrm{d} V / \mathrm{d} \phi)_{0}=(\mathrm{d} V / \mathrm{d} \phi)_{\psi}=0$, where $\psi$ is the amplitude of the positive or negative DL potential step. Both fluid and kinetic theories for DLs, have 


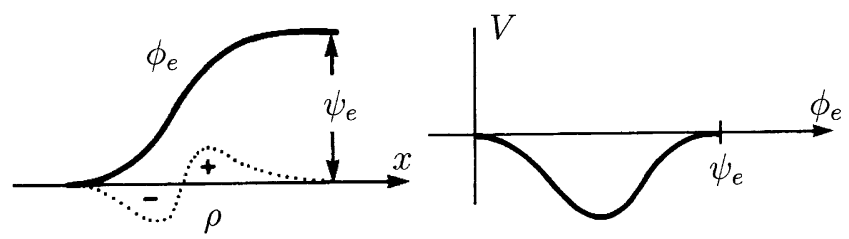

Fig. 8. The electrostatic potential (left) and classical potential (right) for a stationary DL, associated with a dipole-like charge density $\rho$. (After Ref. [25].)

been presented in the past (e.g. Refs. [99-101]). Recently, Newman et al. [102] carried out kinetic 1D simulations of the formation of DLs and electron holes in a current-carrying space plasma. They found that a weak density depression in a current-carrying plasma can lead to the formation of a strong potential ramp (DL).

We now discuss the properties of small-amplitude monotonic DLs. If we assume that the potential $\phi_{\mathrm{e}}=0$ at $\xi=-\infty$ and $\phi_{\mathrm{e}}=\psi_{\mathrm{e}}$ at $\xi=\infty$, and that $n_{\mathrm{e}}=n_{\mathrm{i}}=n_{0}$ at $\phi_{\mathrm{e}}=0$, we then have to leading order [103]

$$
\frac{n_{\mathrm{e}}}{n_{0}}=1+a_{\mathrm{e}} \phi_{\mathrm{e}}-\frac{4}{3} b_{\mathrm{e}} \phi_{\mathrm{e}}^{3 / 2}+\frac{1}{2} G\left(M_{\mathrm{e}}\right) \phi_{\mathrm{e}}^{2}
$$

and

$$
\frac{n_{\mathrm{i}}}{n_{0}}=1-\frac{a_{\mathrm{i}}}{\tau} \phi_{\mathrm{e}}+\frac{4}{3} \frac{b_{\mathrm{i}}}{\tau^{3 / 2}}\left[\psi_{\mathrm{e}}^{3 / 2}-\left(\psi_{\mathrm{e}}-\phi_{\mathrm{e}}\right)^{3 / 2}\right]+\frac{1}{2 \tau^{2}} G\left(M_{\mathrm{i}}\right) \phi_{\mathrm{e}}^{2}-\frac{1}{\tau^{2}}\left[G\left(M_{\mathrm{i}}\right)-a_{\mathrm{i}}^{2}\right] \phi_{\mathrm{e}} \psi_{\mathrm{e}},
$$

where we have kept terms up to order $\phi_{\mathrm{e}}^{2}$, and where $G$ is a monotonic decreasing function which the limits $G(0)=1$ and $G(u)=\exp \left(-u^{2} / 2\right)+3 u^{-4}$ for $|u| \gg 1$. A condition for the DL is that $n_{\mathrm{e}}=n_{\mathrm{i}}$ at $\phi_{\mathrm{e}}=\psi_{\mathrm{e}}$, giving

$$
-\left(a_{\mathrm{e}}+\frac{a_{\mathrm{i}}}{\tau}\right)+\frac{4}{3}\left(b_{\mathrm{e}}+\frac{b_{\mathrm{i}}}{\tau^{3 / 2}}\right) \psi_{\mathrm{e}}^{1 / 2}-\left(C_{1}+C_{2}\right) \psi_{\mathrm{e}}=0
$$

where $C_{1}=(1 / 2)\left[G\left(M_{\mathrm{e}}\right)-G\left(M_{\mathrm{i}}\right) / \tau^{2}\right]$ and $C_{2}=\left[G\left(M_{\mathrm{i}}\right)-a_{\mathrm{i}}^{2}\right] / \tau^{2}$. The particle densities inserted into Poisson's equation and integrated once gives the classical potential

$$
\begin{aligned}
V\left(\phi_{\mathrm{e}} ; a_{\mathrm{e}}, b_{\mathrm{e}}, a_{\mathrm{i}}, b_{\mathrm{i}}, \tau\right) \\
=-\frac{1}{2}\left(a_{\mathrm{e}}+\frac{a_{\mathrm{i}}}{\tau}\right) \phi_{\mathrm{e}}^{2}+\frac{4}{3}\left(\frac{b_{\mathrm{i}}}{\tau^{3 / 2}}\left\{\psi_{\mathrm{e}}^{3 / 2} \phi_{\mathrm{e}}+\frac{2}{5}\left[\left(\psi_{\mathrm{e}}-\phi_{\mathrm{e}}\right)^{5 / 2}-\psi_{\mathrm{e}}^{5 / 2}\right]\right\}+\frac{2}{5} b_{\mathrm{e}} \phi_{\mathrm{e}}^{5 / 2}\right) \\
\quad-\frac{C_{1}}{3} \phi_{\mathrm{e}}^{3}-\frac{C_{2}}{2} \phi_{\mathrm{e}}^{2} \psi_{\mathrm{e}} .
\end{aligned}
$$

The condition $V\left(\psi_{\mathrm{e}} ; a_{\mathrm{e}}, b_{\mathrm{e}}, a_{\mathrm{i}}, b_{\mathrm{i}}, \tau\right)=0$ gives the nonlinear dispersion relation

$$
-\left(a_{\mathrm{e}}+\frac{a_{\mathrm{i}}}{\tau}\right)+\left(\frac{16}{15} b_{\mathrm{e}}+\frac{8}{5} \frac{b_{\mathrm{i}}}{\tau^{3 / 2}}\right) \psi_{\mathrm{e}}^{1 / 2}-\left(\frac{2 C_{1}}{3}+C_{2}\right) \psi_{\mathrm{e}}=0
$$

which, together which Eq. (44) relates the amplitude $\psi_{\mathrm{e}}$ to the velocity (hidden in $a_{\mathrm{e}}, b_{\mathrm{e}}, a_{\mathrm{i}}$ and $b_{\mathrm{i}}$ ). From Eqs. (44) and (46) we have

$$
b_{\mathrm{e}}=\frac{b_{\mathrm{i}}}{\tau^{3 / 2}}+\frac{5}{4} C_{1} \psi_{\mathrm{e}}^{1 / 2}
$$

and

$$
-\left(a_{\mathrm{e}}+\frac{a_{\mathrm{i}}}{\tau}\right)=-\frac{8}{3} \frac{b_{\mathrm{i}}}{\tau^{3 / 2}} \psi_{\mathrm{e}}^{1 / 2}+\left(C_{2}-\frac{2}{3} C_{1}\right) \psi_{\mathrm{e}}
$$




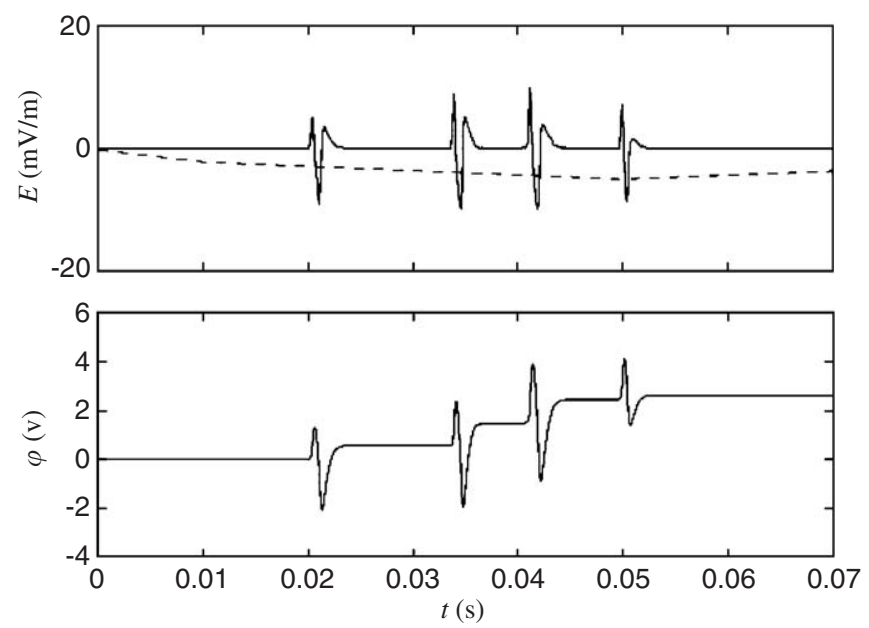

Fig. 9. The electric field (upper panel) and potential (lower panel) of combined DL and localized potential maxima/minima, observed by the Cluster spacecraft. (After Ref. [55].)

which inserted into Eq. (45) gives the classical potential

$$
\begin{aligned}
V\left(\phi_{\mathrm{e}} ; a_{\mathrm{e}}, b_{\mathrm{e}}, a_{\mathrm{i}}, b_{\mathrm{i}}, \tau\right)= & \frac{4}{3} \frac{b_{\mathrm{i}}}{\tau^{3 / 2}}\left\{\phi_{\mathrm{e}} \psi_{\mathrm{e}}^{1 / 2}\left(\psi_{\mathrm{e}}-\phi_{\mathrm{e}}\right)+\frac{2}{5}\left[\left(\psi_{\mathrm{e}}-\phi_{\mathrm{e}}\right)^{5 / 2}-\psi_{\mathrm{e}}^{5 / 2}+\phi_{\mathrm{e}}^{5 / 2}\right]\right\} \\
& -\frac{C_{1}}{3} \phi_{\mathrm{e}}^{2}\left(\psi_{\mathrm{e}}^{1 / 2}-\phi_{\mathrm{e}}^{1 / 2}\right)^{2},
\end{aligned}
$$

where we note that the terms multiplied by $C_{2}$ cancel. In the small amplitude limit $\psi \ll b_{\mathrm{i}} / \tau^{3 / 2}$, all terms involving $C_{1}$ and $C_{2}$ vanish from the equations, and we have from Eqs. (47) and (48) the conditions $b_{\mathrm{e}}=b_{\mathrm{i}} / \tau^{3 / 2}$ and $a_{\mathrm{e}}+a_{\mathrm{i}} / \tau=$ $(8 / 3) b_{\mathrm{i}} \psi^{1 / 2} / \tau^{3 / 2}$ for the existence of a DL. On the other hand, if the factor $b_{\mathrm{i}} / \tau^{3 / 2} \ll \psi$ so that the first term of Eq. (49) can be neglected (and only the higher-order term remains), then we can integrate the energy equation to obtain [101]

$$
\phi_{\mathrm{e}}=\frac{1}{4} \psi_{\mathrm{e}}[1+\tanh (\kappa \xi)]^{2}
$$

where $\kappa=\left(C_{1} \psi_{\mathrm{e}} / 24\right)^{1 / 2}$.

\subsection{Bipolar potential pulses}

Recently, a new type of electrostatic solitary waves has been recorded in the solar wind $[104,105]$, featuring a tripolar electric field signal. The electric field in such isolated electrostatic structures is predominantly aligned with the solar wind magnetic field, with a spatial width of the order $\sim 25 r_{\text {De }}$. There is a small but finite electric potential drop across the structure, implying an average electric field generally directed away from the Sun. In that respect they are similar, although with a smaller amplitude, to the weak DLs observed in the auroral region, and to the electrostatic solitary waves observed in the other regions of the magnetosphere. More detailed observations of tripolar solitary structures have been recorded in the auroral zone by the Cluster multi-spacecraft mission [55]; see Fig. 9. They revealed a simultaneous occurrence of bipolar and tripolar pulses, the latter being somewhat less frequent. Their duration is $1-3 \mathrm{~ms}$, which is $\sim 50 \%$ longer than that of dipoles. Integration over the electric field of these tripolar pulses yields a measurable potential change of about $2.5 \mathrm{~V}$ across all four solitary waves, consistent with weak or hybrid DLs. On several occasions correlated observations of the trains of tripoles (up to four of them) were recorded by two or more of the Cluster spacecrafts.

Within the phase-space vortex model, the WIND and Cluster tripolar pulses whose potential signatures exhibit an overall potential drop coupled with a potential hump and dip as opposed to just one hump or one dip in the case of bipolar pulses, would require both trapped electrons and trapped ions to be sustained. Conversely, DLs that feature a localized potential jump similar to that of a tripole can be described in simple terms as the special case of infinitely 
stretched electron and ion holes [106,25], and as a consequence they must be supported by a non-Maxwellian particle distribution at least on one side. While such behavior at the far boundary can be relatively easily realized in a laboratory experiment by the injection of an appropriate particle beam, it is not clear whether such restrictive asymptotic boundary conditions are always present in the magnetospheric and interplanetary plasmas, where the tripolar structures were recorded. Some attempts have been made to model the tripolar electric signals theoretically in the small-amplitude limit [107], but their full explanation is still an open issue.

\section{Dynamics of electron and ion holes}

Stationary solutions of the electron and ion holes feature the final state of unstable fluctuations that are triggered by free energy sources (viz. the electron and ion beams) in plasmas. The spatio-temporal evolution of the electron and ion holes in plasmas can be investigated by means of computer simulations. As we will see below, the nonlinear dynamics of the electron and ion holes is rather intriguing and interesting.

\subsection{Self-acceleration of electron holes}

Let us discuss the numerical results for the dynamics of non-relativistic electron holes in an electron-ion plasma [108]. In order to investigate the dynamics of electron holes numerically in a controlled manner, we use Schamel's solution of the stationary Vlasov-Poisson system with immobile ions to construct initial conditions for our simulations. Accordingly, we use Eqs. (9) and (10) for the ion and electron density, respectively, in Poisson's equation (5) which is solved numerically to obtain the electrostatic potential, which is then used in Eq. (8) to construct the initial condition for the electron distribution function. In Fig. 10, we have plotted the electric potential and electron number density $N_{\mathrm{e}} \equiv n_{\mathrm{e}} / n_{0}$ of the electron hole for the case of a fixed ion background, viz. $n_{\mathrm{i}}=n_{0}$. We note that larger values of $M_{\mathrm{e}}$ and $\left|\beta_{\mathrm{e}}\right|$ give smaller maxima of the potential and less deep electron density minima, in agreement with Fig. 3(a) of Ref. [26]. The potentials obtained in Fig. 10 are used to construct the numerical initial conditions for the electron distribution function (8) of the electron holes, to be used in our Vlasov simulations including the ion dynamics. We are considering a plasma consisting of electrons and oxygen ions, which is common in the Earth's ionosphere. In the simulations, the Vlasov-Poisson system (1)-(2) is solved numerically, where the ions are assumed to initially form a homogeneous Maxwellian background, $f_{\mathrm{i}}=\left[n_{0} /(2 \pi)^{1 / 2} V_{T i}\right] \exp \left(-v^{2} / 2 V_{T i}^{2}\right)$, with $T_{\mathrm{i}}=T_{\mathrm{e}}$ and $m_{\mathrm{i}} / m_{\mathrm{e}}=29,500$. As an initial condition for the electron distribution function, we use the parameters $M_{\mathrm{e}}=0$ and $\beta_{\mathrm{e}}=-0.7$ (the solid lines in Fig. 10) for an electron hole initially at rest. Since the electron hole potential is positive, it will accelerate ions away from the electron hole, thus creating an ion cavity at the electron hole. In order to study the stability properties of the electron hole, we introduce a small perturbation of the electron density at the electron hole, which will work as a seed for any instability. In natural plasmas, waves and streams occur naturally due to turbulent processes, while
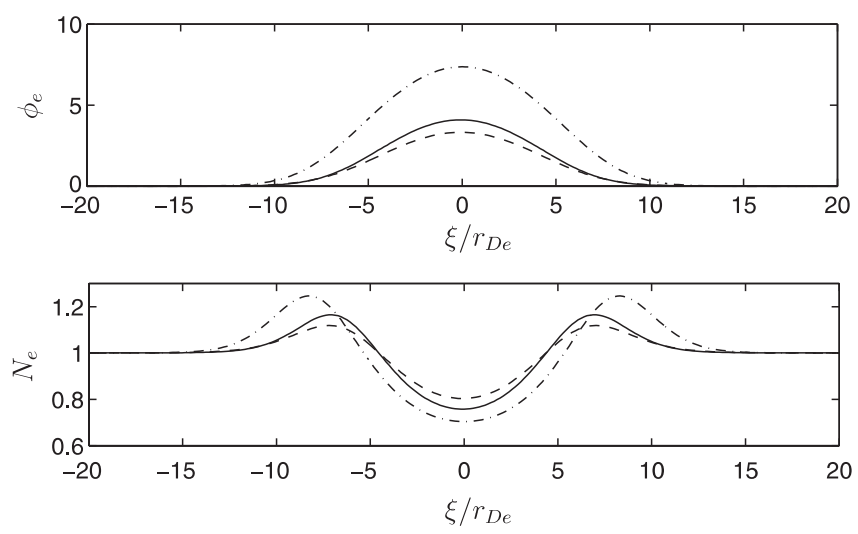

Fig. 10. The potential (upper panel) and the electron density $N_{\mathrm{e}}=n_{\mathrm{e}} / n_{0}$ (lower panel), associated with a standing electron hole $\left(M_{\mathrm{e}}=0\right)$ with $\beta_{\mathrm{e}}=-0.7$ (solid lines) and $\beta_{\mathrm{e}}=-0.5$ (dash-dotted lines), and a moving electron hole with $M_{\mathrm{e}}=0.5$ and $\beta_{\mathrm{e}}=-0.7$ (dashed lines) in plasmas with fixed ion background $\left(N_{\mathrm{i}}=1\right)$. (After Ref. [108].) 

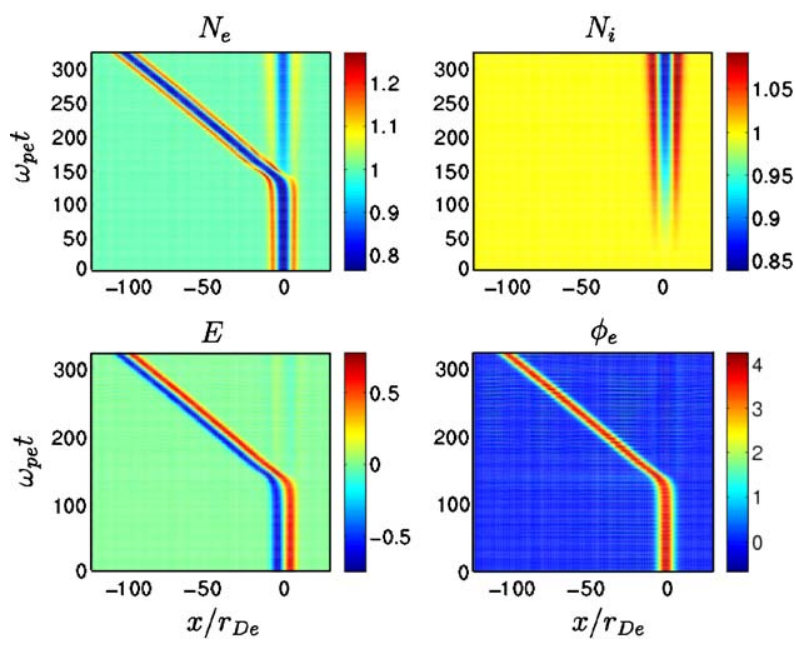

Fig. 11. The electron density $N_{\mathrm{e}}=n_{\mathrm{e}} / n_{0}$ (upper left panel), ion density $N_{\mathrm{i}}=n_{\mathrm{i}} / n_{0}$ (upper right panel), electric field (lower left panel) and potential (lower right panel) of an initially standing electron hole. The chosen trapping parameter is $\beta_{\mathrm{e}}=-0.7$. (After Ref. [108].)
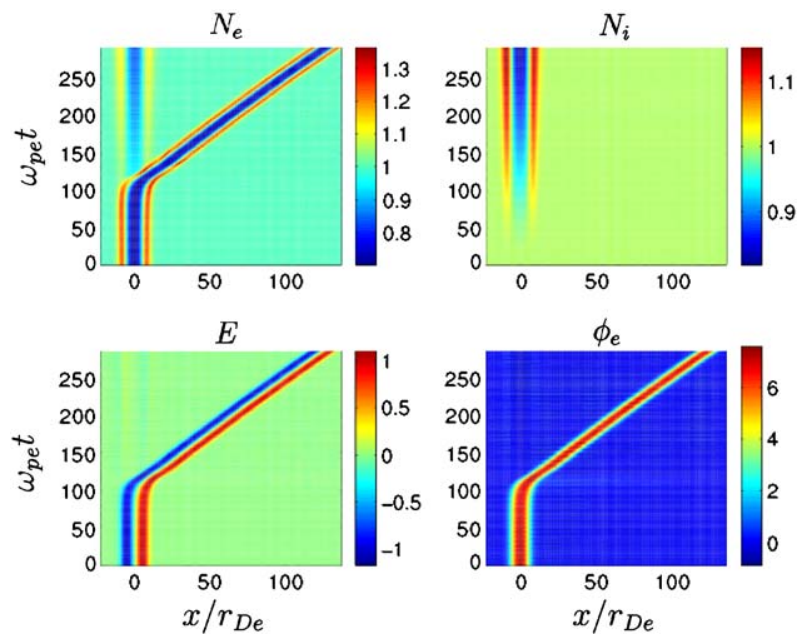

Fig. 12. The electron density (upper left panel), the ion density (upper right panel), the electric field (lower left panel) and the potential (lower right panel) of an initially stationary electron hole. The chosen trapping parameter is $\beta_{\mathrm{e}}=-0.5$ (after Ref. [108]).

in the simulation, we introduce the perturbation in a controlled manner. The perturbation is taken to be a Maxwellian population of electrons added to the initial condition for the electron hole, with the same temperature as the background electrons and with the density perturbation of the form $\delta n_{\mathrm{e}} / n_{0}=-0.008 \sinh \left(x / 2 r_{\text {De }}\right) / \cosh ^{2}\left(x / 2 r_{\text {De }}\right)$. In Fig. 11 we display the time development of the electron and ion particle densities together with the potential and electric field (the latter normalized by $r_{\mathrm{De}} k_{\mathrm{B}} T_{\mathrm{e}} / e$ ). The most striking feature in the simulation, seen at time $t \approx 130 \omega_{\mathrm{pe}}^{-1}$, is that the electron hole "suddenly" starts moving in the negative $x$ direction with a Mach number $M_{\mathrm{e}} \approx 0.55$. The transition happens when the ion density has formed a deep enough cavity, turning the equilibrium of the electron hole into an unstable state. The direction of the propagation is sensitive to the small perturbation in the initial condition. This situation is similar to the classical example of a ball balanced at the top of a hill: Only a very small push in any direction is needed for the ball to start accelerating and rolling downhill. When the electron hole has escaped the ion cavity, the ion density cavity continues to deepen and an electron density cavity is created at the same place, neutralizing the plasma. In Fig. 12, we have repeated the same numerical experiment as in Fig. 11, but with $\beta_{\mathrm{e}}=-0.5$, making the electron hole larger. We have also included the same local perturbation of the plasma as the one used in Fig. 11. In this 

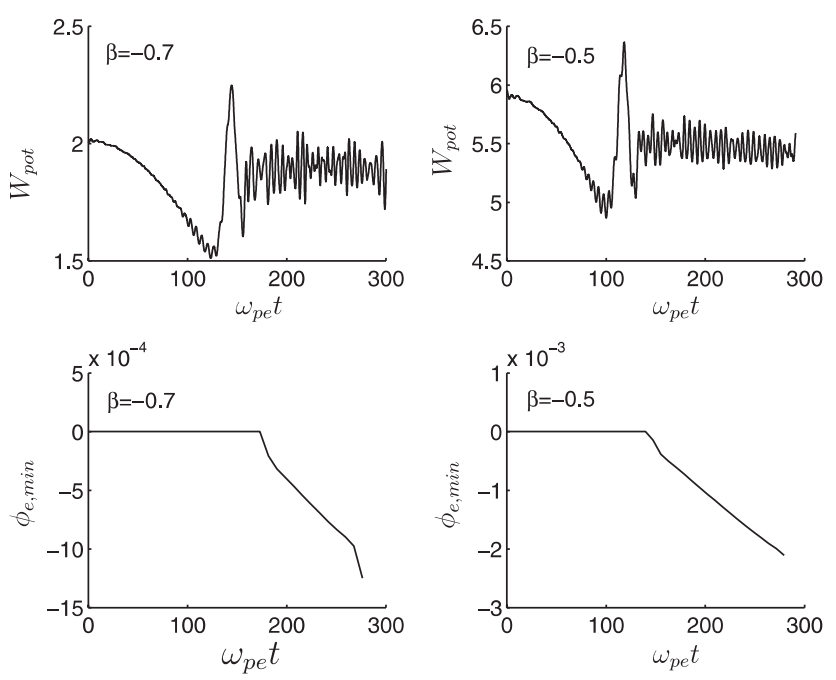

Fig. 13. The total potential energy $W_{\text {pot }}=(1 / 2) \int E^{2} \mathrm{~d} x$ as a function of time (upper panels) and the minimum of the electrostatic potential (lower panels) for $\beta_{\mathrm{e}}=-0.7$ (left panels) and $\beta_{\mathrm{e}}=-0.5$ (right panels), obtained from the model for a stationary electron hole in the presence of a varying ion density, where the ion density is obtained from the Vlasov simulations (after Ref. [108]).

case, the electron hole starts moving in the positive $x$ direction at $t \approx 100 \omega_{\mathrm{pe}}^{-1}$, also with a Mach number $M_{\mathrm{e}} \approx 0.55$. For both $\beta_{\mathrm{e}}=-0.7$ and $\beta_{\mathrm{e}}=-0.5$, the potential maximum decreases slightly during the transition from standing to moving electron holes, from $\psi_{\mathrm{e}}=4.5$ to 3.8 and from $\psi_{\mathrm{e}}=7.8$ to 7.0 , respectively. For $M_{\mathrm{e}}=0.55$, the diagram in Fig. 3(a) of Ref. [26] predicts that the trapping parameter has changed to $\beta_{\mathrm{e}} \approx-0.67$ and $\beta_{\mathrm{e}} \approx-0.45$, respectively, for the two cases. We observe a difference in the transition time of the two holes: The transition of the hole with $\beta_{\mathrm{e}}=-0.7$ occurs at $t \approx 130$, while for the larger hole with $\beta_{\mathrm{e}}=-0.5$, it occurs at an earlier time $t \approx 100$. This may be due to the larger potential and electric field of the electron hole with $\beta_{\mathrm{e}}=-0.5$, resulting in a faster acceleration of the ions and an earlier onset of the acceleration of the electron hole. A parametric study of this phenomenon is left for future research. We next analyze the distribution of energy in the system. The total energy is conserved exactly in the continuous system and to a high degree in our numerical simulations, and is distributed between the kinetic energy of the particles and the potential energy stored in the electric field. In the upper panel of Fig. 13, we have plotted the potential energy $W_{\text {pot }}=(1 / 2) \int E^{2} \mathrm{~d} x$ of the system for the two simulation runs, as a function of time. Here, most of the potential energy is stored in the large-amplitude bipolar electric field of the electron hole, while some potential energy is released in high-frequency Langmuir waves. We observe a gradual decrease of the potential energy till the transition time when the electron hole leaves the ion cavity and the potential energy performs large fluctuations $\left(t \approx 130 \omega_{\mathrm{pe}}^{-1}\right.$ for the $\beta_{\mathrm{e}}=-0.7$ case and $t \approx 100 \omega_{\mathrm{pe}}^{-1}$ for the $\beta_{\mathrm{e}}=-0.5$ case), after which the potential energy performs high-frequency oscillations attributed to Langmuir waves released in the transition, around a somewhat smaller constant $W_{\text {pot }}$ attributed to the propagating electron hole bipolar electric field. The slow decrease of the potential energy in the initial phase indicates that the positive electrostatic potential of the electron hole accelerates the ions which leave the vicinity of the electron hole. In a homogeneous ion background, the single, steady-state electron hole is associated with a positive potential which traps electrons. Since the positive potential of the electron hole and the negative potential of the ion cavity are competing processes, there could be a problem of the existence of a stationary electron hole if the ion density becomes deep enough. In our case, the electron hole remains stable but escapes the ion cavity. In the lower panel of Fig. 13, we have taken the ion density $n_{\mathrm{i}}$ obtained in our Vlasov simulation as an input to Poisson's equation, which we then solve with the electron density given by Eq. (10). The potential of the electron hole is normally positive everywhere when the ions are fixed background. In the presence of a local ion density cavity, the potential may have a slightly negative minimum, and at this point we prescribe a Maxwellian distribution for the untrapped electrons in the same manner as in e.g. Refs. [22,25]. We plotted the potential minimum $\phi_{\min }$ as a function of time, and found that at $t \approx 170 \omega_{\mathrm{pe}}^{-1}$ a part of the potential becomes negative. It seems that the acceleration of the electron hole occurs at approximately the same time as a part of the potential for the theoretical model becomes negative. An alternative way of explaining accelerating 

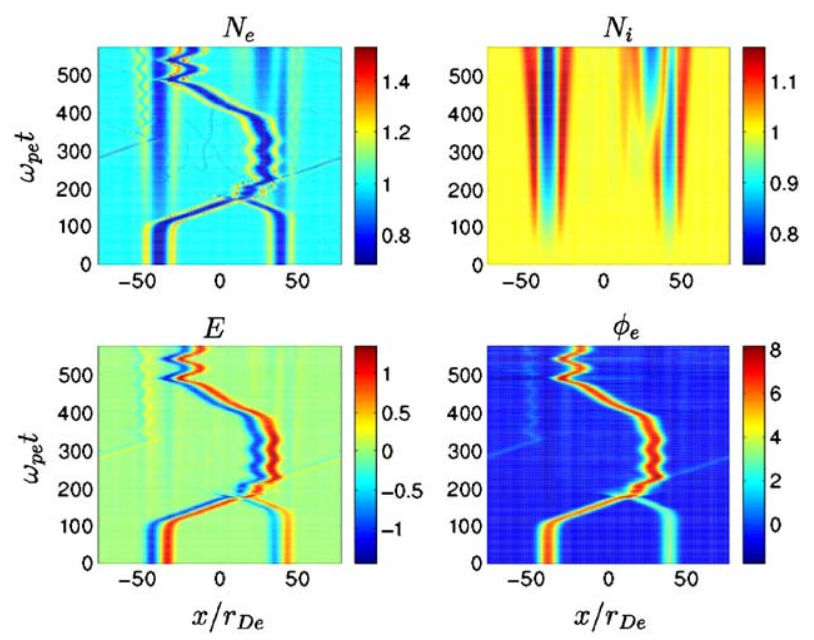

Fig. 14. The electron density (upper left panel), the ion density (upper right panel), the electric field (lower left panel), and the potential (lower right panel) of two electron holes initially placed at $x / r_{\mathrm{De}}= \pm 40$. The trapping parameter $\beta_{\mathrm{e}}=-0.5$ for the left electron hole initially placed at $x / r_{\mathrm{De}}=-40$, and $\beta_{\mathrm{e}}=-0.7$ for the right electron hole placed at $x / r_{\mathrm{De}}=40$ (after Ref. [108]).
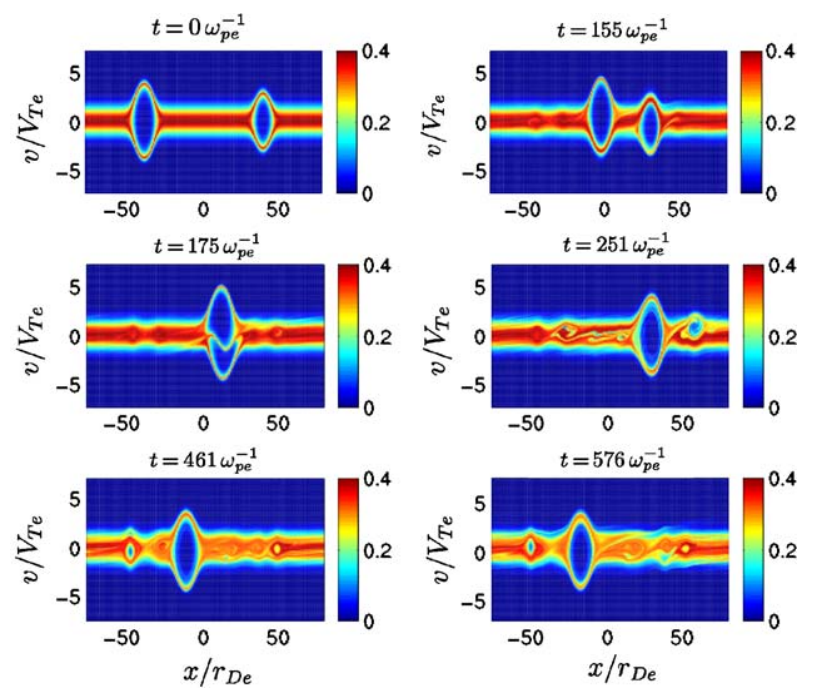

Fig. 15. The electron distribution for two electron holes at $t=0 \omega_{\mathrm{pe}}^{-1}$ (upper left panel), $t=155 \omega_{\mathrm{pe}}^{-1}$ (upper right panel), $t=175 \omega_{\mathrm{pe}}^{-1}$ (middle left panel), $t=251 \omega_{\mathrm{pe}}^{-1}$ (middle right panel), $t=461 \omega_{\mathrm{pe}}^{-1}$ (lower left panel) and $t=576 \omega_{\mathrm{pe}}^{-1}$ (lower right panel). See the associated densities, etc. in Fig. 14. Initially, the left electron hole (placed at $x / r_{\mathrm{De}}=-40$ ) has a trapping parameter $\beta_{\mathrm{e}}=-0.5$, while the right electron hole (placed at $\left.x / r_{\mathrm{De}}=40\right)$ has $\beta_{\mathrm{e}}=-0.7$ (after Ref. [108]).

electron holes is as follows: The positive electron hole potential starts to reflect low energy ions giving rise to a local reduction of $N_{\mathrm{i}}$. After a while, at the center of the electron hole, the condition $r_{\mathrm{De}}^{2} \phi_{\mathrm{e}}^{\prime \prime}(x)=N_{\mathrm{e}}(x)-N_{\mathrm{i}}(x)<0$ for $x$ near zero is no longer met and the standing structure ceases to exist. Then the electron hole is accelerated such that a free ion component exists, giving rise to a free ion density $N_{\text {if }}(x)$ necessary for the maintenance of the inequality near the electron hole center. Fig. 11 indicates that indeed $N_{\mathrm{i}}(x)=N_{\mathrm{if}}(x) \approx 1$ at the location of the propagating electron hole.

Finally, we display results for nonlinear interactions between two electron holes with each other and with ions in a longer simulation; see Figs. 14 and 15 . The electron holes with $\beta_{\mathrm{e}}=-0.5$ and -0.7 were initially placed at $x=-40 r_{\text {De }}$ and $40 r_{\text {De }}$, respectively. A local electron density perturbation was taken to be Maxwellian with the density $\delta n_{\mathrm{e}} / n_{0}=-0.08\left\{\sinh \left[\left(x / r_{\mathrm{De}}-40\right) / 2\right] / \cosh ^{2}\left[\left(x / r_{\mathrm{De}}-40\right) / 2\right]+\sinh \left[\left(x / r_{\mathrm{De}}+40\right) / 2\right] / \cosh ^{2}\left[\left(x / r_{\mathrm{De}}+40\right) / 2\right]\right\}$, i.e. 
the same perturbations as in the single-hole cases, centered at the two electron holes. Here, the electron holes also create local ion density cavities, and after some time escapes the density cavities at the same times as in the single electron hole cases. This can clearly be seen in Fig. 14, where the two electron holes start moving at $t \approx 100 \omega_{\mathrm{pe}}^{-1}$ and $t \approx 130 \omega_{\mathrm{pe}}^{-1}$, respectively. At $t \approx 170 \omega_{\mathrm{pe}}^{-1}$, the two electron holes collide inelastically and merge into a new electron hole, in accordance with the results of Ref. [46], after which the single electron hole propagates slightly in the positive $x$ direction, and becomes trapped at a local ion density maximum at $x \approx 30 r_{\text {De }}$; see the upper right panel of Fig. 14 for the ion density and the lower right panel for the electron hole potential. After $t \approx 400 \omega_{\text {pe }}^{-1}$, a new ion density cavity is created where the electron hole is centered, and at this time the electron hole is again accelerated, now in the negative $x$ direction. At $t \approx 480 \omega_{\mathrm{pe}}^{-1}$, the moving electron hole again encounters an ion density maximum located at $x \approx-30 r_{\text {De }}$, where the electron hole is trapped, performing large oscillations. The electron phase-space density is depicted in Fig. 15: The initial condition (upper left panel), the two electron holes having started moving (upper right panel), collisions between the two electron holes (middle left panel), the newly created electron hole trapped at $x=30 r_{\text {De }}$ (middle right panel), and the electron hole trapped at $x=-30 r_{\text {De }}$ (lower panels). We see that the electron holes remain stable during the acceleration by the ion density cavities.

Poisson's equation (5) was solved for $\phi_{\mathrm{e}}$ as a nonlinear boundary value problem where $\phi_{\mathrm{e}}$ is set to zero far away on each side of the electron hole; a central difference approximation is used for the second derivative in Poisson's equation, leading to a system of nonlinear equations, which is solved iteratively with (a slightly modified) Newton's method. The numerical solutions of the Vlasov-Poisson system were performed with a Fourier method [109]. We used 500 intervals in $x$ space with the domain $-80 \leqslant x / r_{\text {De }} \leqslant 80$, and 300 intervals in velocity space. The electron velocity interval was set to $-15.7 \leqslant v / V_{T e} \leqslant 15.7$ and the ion velocity interval was set to $-0.118 \leqslant v / V_{T e} \leqslant 0.118$. The time step $\Delta t \approx 0.013 \omega_{\mathrm{pe}}^{-1}$ was adapted dynamically to maintain numerical stability.

\subsection{Colliding ion holes}

We now discuss our simulation results for some kinetic effects associated with nonlinear interactions between ion holes and the background electron-ion plasma [110]. Since ion holes are associated with a negative electrostatic potential, they repel electrons. Therefore, low-energy electrons can be trapped between two ion holes, and during collisions between the ion holes, the low-energy electrons are accelerated to high energies by the ion hole potential, forming electron streams escaping the two colliding ion holes. Streams of electrons can excite high-frequency Langmuir waves due to a streaming instability, and thus the processes on the slower ion timescale gives rise to high-frequency waves on an electron timescale. On the other hand, ion holes are characterized by a depletion of the electrons, which gives the possibility that high-frequency Langmuir waves can be trapped in the ion hole. Clearly, if the Langmuir waves have large amplitudes, there will be a modification of the ion hole due to the ponderomotive force acting on the electrons, which are repelled in the direction of decreasing Langmuir wave electric field amplitudes. These electrons then change the ion hole potential, as described via Poisson's equation. Thus, the nonlinear interaction between the ion holes with the background plasma gives rise to a multitude of complex phenomena on different timescales.

In order to investigate the time-dependent dynamics of ion holes as well as kinetic effects for the electrons, we have numerically solved the Vlasov-Poisson system of Eqs. (1) and (2). The initial conditions for $f_{\mathrm{i}}$ is taken as Schamel's distribution [25] for the free and trapped ions, which in the rest frame of the bulk plasma it is given by Eq. (28). We use [111] $m_{\mathrm{i}} / m_{\mathrm{e}}=1836, \beta_{\mathrm{i}}=-1.0$, and $T_{\mathrm{e}} / T_{\mathrm{i}}=10$. The initial condition for the electron distribution function is taken to be the Maxwell-Boltzmann distribution, given by Eq. (29). The potential $\varphi$ for the initial conditions (28) and (29) is obtained by inserting them into Eq. (5), which is then integrated [26,111] to obtain the potential $\varphi$ as a function of $x$; in practice the integration of Eq. (5) is performed numerically. The potential thus obtained is inserted into Eqs. (28) and (29) to obtain the initial conditions for the ion and electron distribution functions.

We present the results for the process of two colliding ion holes, as shown in Figs. 16-18. For the initial conditions, we have assumed that the ion holes initially are well separated in space, and that the interaction between the ion holes is weak, so that the solutions for the single ion holes can be matched in space to form an initial condition for the case with two ion holes. Fig. 16 displays the features of the ion and electron distribution functions for two colliding ion holes, where initially (upper panels) the left ion hole propagates with the speed $u_{0}=0.9 V_{T i}$, and the right ion hole is standing. The ion and electron distribution functions associated with the ion holes are shown before collision at times 

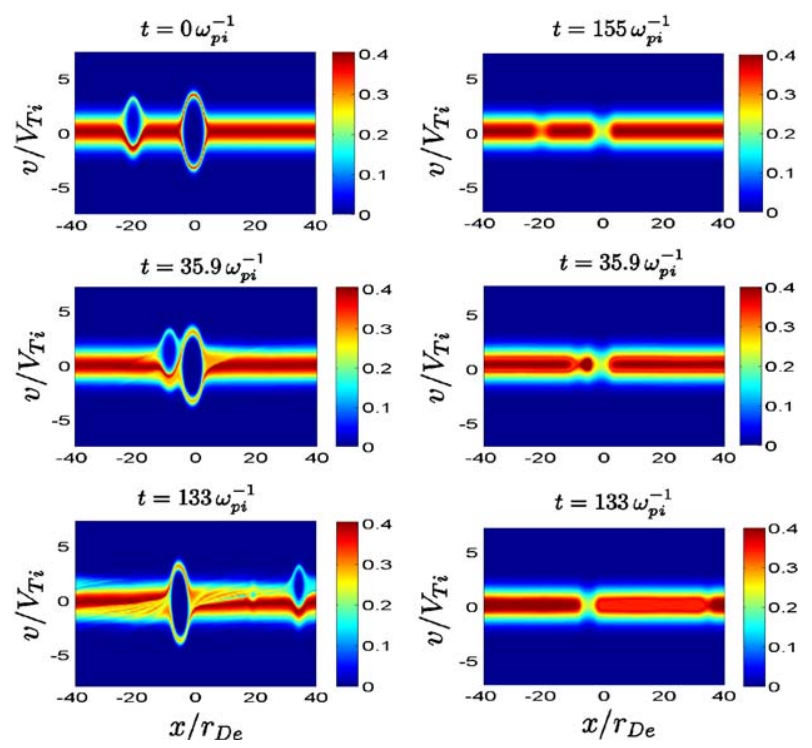

Fig. 16. The normalized ion distribution function $f_{\mathrm{i}} V_{T i} / n_{0}$ (left panels) and electron distribution function $f_{\mathrm{e}} V_{T e} / n_{0}$ (right panels) of two colliding ion holes, before the collision at times $t=0 \omega_{\mathrm{pi}}^{-1}$ (upper panel) and $t=35.9 \omega_{\mathrm{pi}}^{-1}$ (middle panel), and after the collision at $t=133 \omega_{\mathrm{pi}}^{-1}($ lower panel) (after Ref. [110])
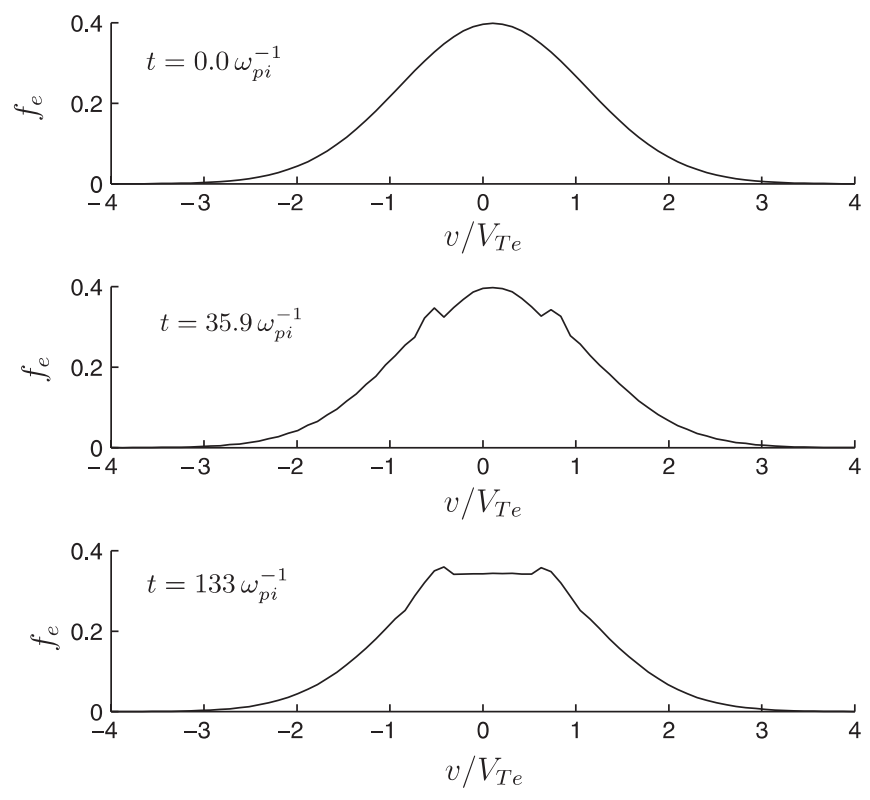

Fig. 17. The electron velocity distribution (normalized by $n_{0} / V_{T e}$ ) at $x=8 r_{\text {De }}$, for $t=0 \omega_{\mathrm{pi}}^{-1}$ (upper panel), $t=35.9 \omega_{\mathrm{pi}}^{-1}$ (middle panel) and $t=133 \omega_{\mathrm{pi}}^{-1}$ (lower panel) (after Ref. [110]).

$t=0 \omega_{\mathrm{pi}}^{-1}$ (upper panels) and $t=35.9 \omega_{\mathrm{pi}}^{-1}$ (middle panels), and after collision at time $t=133 \omega_{\mathrm{pi}}^{-1}$ (lower panels). The left panels of Fig. 16 exhibit that the two ion holes undergo collisions without being destroyed, and as can be seen in the right panels of Fig. 16, the electrons have a strongly non-Maxwellian flat top distribution in the region between the ion holes after that the collision has taken place. We have plotted the electron velocity distribution function against $v / V_{T e}$ at $x=8.0 r_{\mathrm{D}}$ in Fig. 17. We see that the initial Maxwellian distribution (the upper panel) changes to 

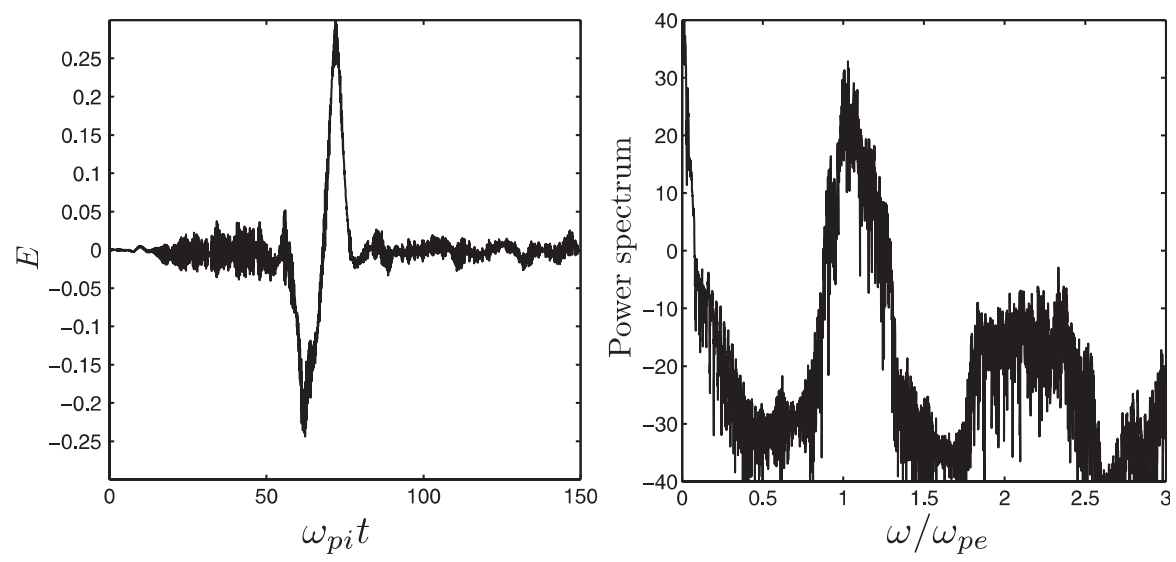

Fig. 18. The electric field at $x=8.0 r_{\text {De }}$ as a function of $\omega_{\text {pi }} t$ (left panel) and the power spectrum of the electric field as a function of $\omega / \omega_{\text {pe }}($ right panel) (after Ref. [110]).

a distribution with beams at $v \approx \pm 0.6 V_{T e}$ (the middle panel) slightly before collision, and to a flat-top distribution with two maxima after collision (the lower panel). The reason for the creation of the flat-topped velocity distribution is that the two ion holes are associated with negative electrostatic potentials, and the electrons entering the region between the ion holes after collision must have a large enough kinetic energy to cross the potential barriers that are set up by the ion holes. The flattening of the low-velocity region then occurs probably due to repeated reflections and slowing down of the trapped electrons against the two moving potential barriers. On the other hand, we also observe that before collision, the low-energy population of electrons are compressed between the ion holes (see the middle right panel of Fig. 16) and released during collision; these electrons are then accelerated to form beams of electrons (see the middle panel of Fig. 17) which escape the ion holes. The energy of the electron beams are then released in a beam-plasma instability triggering high-frequency plasma oscillations, as illustrated in Fig. 18 (the left panel) where the electric field at $x=8.0 r_{\mathrm{De}}$ is plotted as a function of $\omega_{\mathrm{pi}} t$. High-frequency plasma oscillations are beginning to be excited at time $t \approx 10 \omega_{\mathrm{pi}}^{-1}$. The large-amplitude bipolar electric fields of the ion hole appear as it crosses $x=8.0 r_{\mathrm{De}}$ at time $t \approx 50-80 \omega_{\mathrm{pi}}^{-1}$. By examining the frequency spectrum of the electric field (the right panel), we notice that the high-frequency oscillations have broad frequency spectrum which is upshifted compared to the electron plasma frequency.

The numerical solutions of the Vlasov-Poisson system were performed by means of a Fourier transform method [109]. We used 500 intervals in $x$ space with the domain $-40 \leqslant x / r_{\text {De }} \leqslant 40$ with periodic boundary conditions. We used 300 intervals in velocity space. The electron and ion speed intervals were in the ranges $-15.7 \leqslant v / V_{T e} \leqslant 15.7$ and $-0.118 \leqslant v / V_{T e} \leqslant 0.118$, respectively. The timestep $\Delta t \approx 0.013 \omega_{\mathrm{pe}}^{-1}$ was adapted dynamically to maintain numerical stability.

\subsection{Phase-space holes in pair plasmas}

In the preceding sections, we have discussed kinetic structures in an electron-ion plasma where the masses of electrons and ions are quite distinct. However, there are situations where the masses of plasma particles could be identical, but their charges different. Such pair plasmas [112-114,80] are ubiquitous in semiconductors in the form of electron and ion holes, in many astrophysical environments (from pulsars to quasars), as well as in the early universe and in active galactic nuclei, in our own galaxy and in supernova remnants in the form of electrons and positrons [115]. Laboratory astrophysics studies [116] involving super strong laser pulses may also encounter electrons and positrons which could be created by accelerated electrons in intense wakefields [117]. Electron-positron pair production is expected to occur in post-disruption plasmas in large tokamaks [118], as well as in next generation laser-plasma interaction experiments where quantum electrodynamic processes become important [119]. On the other hand, a series of laboratory experiments have recently been conducted in which a pair-ion plasma is created by impact ionization of a gas of fullerene $\mathrm{C}_{60}$ carbon nanotubes [120]. By magnetic filtering of the plasma, electrons and ions are removed and 
an almost pure ion plasma, consisting of singly charged $\mathrm{C}_{60}^{+}$and $\mathrm{C}_{60}^{-}$ions is created. This poses a unique possibility to investigate the collective behavior [120] of a pair-ion plasma experimentally under controlled conditions. A natural product of large-amplitude electrostatic waves in plasmas is the creation of phase-space holes, in which a population of the particles is trapped in self-created localized electric potentials. A theory for small-amplitude periodic holes and DLs in a pair plasma has been developed by Schamel and Luque [121].

In the following, we discuss a new aspect of large amplitude solitary phase-space holes in an ion plasma composed of positive and negative ions without electrons [122]. Our theoretical model is based on solutions of the stationary Vlasov-Poisson equations for phase-space holes, which are characterized by a population of negatively charged ions in a self-created localized positive electrostatic potential hump, and reflected positive ions on each side of the potential maximum. We emphasize that by analogy, the theory also applies to phase-space holes in which positively charged ions are trapped in localized negative potential wells, while negative ions are reflected on each side of the potential minimum. Furthermore, we also present computer simulations of the time-dependent Vlasov-Poisson equations in order to understand the complex dynamics of nonlinearly interacting phase-space holes in a pair-ion plasma. It should be stressed that the underlying physics of propagating ion holes in pair-ion plasma (with equal ion temperatures and non-isothermal distributions for the two ion species having the same mass) is significantly different from ion holes in an electron-ion plasma [25,20], where one assumes non-isothermal ions and Boltzmann distributed electrons. Here, the two species must be treated kinetically on an equal footing and thus the Vlasov equation must be solved for both particle species.

In our pair plasma the positively and negatively charged particles are designated by the superscripts ${ }^{+}$and ${ }^{-}$, respectively. The positive (negative) particle has a charge $q^{+}=Z^{+} e\left(q^{-}=-Z^{-} e\right)$, where $Z^{+}\left(Z^{-}\right)$is the charge state. The dynamics of a collisionless plasma is governed by the Vlasov equation for the positively and negatively charged ions, i.e.

$$
\frac{\partial f^{+}}{\partial t}+v \frac{\partial f^{+}}{\partial x}-\frac{1}{\mathscr{M}} \frac{\partial \phi}{\partial x} \frac{\partial f^{+}}{\partial v}=0
$$

and

$$
\frac{\partial f^{-}}{\partial t}+v \frac{\partial f^{-}}{\partial x}+\frac{\partial \phi}{\partial x} \frac{\partial f^{-}}{\partial v}=0
$$

respectively, where $\mathscr{M}=Z^{-} m^{+} / Z^{+} m^{-}$. The distribution functions for the positively and negatively charged ions $f^{+}$and $f^{-}$have been normalized by $n_{0} / V_{\mathrm{T}}$ and $Z^{-} n_{0} / Z^{+} V_{\mathrm{T}}$, respectively, the time $t$ by $\omega_{\mathrm{p}}^{-1}$, the space $x$ by $r_{\mathrm{D}}$, the velocity $v$ by $V_{\mathrm{T}}$, and the potential $\phi$ by $k_{\mathrm{B}} T^{-} / Z^{-} e$. Here $m^{+}\left(m^{-}\right)$is the positive (negative) ion mass, $n_{0}$ is the equilibrium particle density of negative ions, $V_{\mathrm{T}}=\left(k_{\mathrm{B}} T^{-} / m^{-}\right)^{1 / 2}$ is the thermal speed, $T^{-}$is the temperature, $\omega_{\mathrm{p}}=\left[n_{0}\left(Z^{-}\right)^{2} e^{2} / \epsilon_{0} m^{-}\right]^{1 / 2}$ is the plasma frequency, and $r_{\mathrm{D}}=V_{\mathrm{T}} / \omega_{\mathrm{p}}$. The system is closed by Poisson's equation

$$
\frac{\partial^{2} \phi}{\partial x^{2}}=n^{-}-n^{+}
$$

where $n^{-}=\int_{-\infty}^{\infty} f^{-} \mathrm{d} v$ and $n^{+}=\int_{-\infty}^{\infty} f^{+} \mathrm{d} v$.

We now assume quasi-stationary structures moving with a constant speed $u_{0}$ relative to the bulk plasma. Accordingly, we assume that $f^{+}$and $f^{-}$only depends on $v$ and $\xi=x-u_{0} t$, while $\phi$ only depends on $\xi$. With this ansatz, we have from Eqs. (51), (52) and Poisson's equation (53), respectively,

$$
\begin{aligned}
& \left(-u_{0}+v\right) \frac{\partial f^{+}}{\partial \xi}-\frac{1}{\mathscr{M}} \frac{\mathrm{d} \phi}{\mathrm{d} \xi} \frac{\partial f^{+}}{\partial v}=0 \\
& \left(-u_{0}+v\right) \frac{\partial f^{-}}{\partial \xi}+\frac{\mathrm{d} \phi}{\mathrm{d} \xi} \frac{\partial f^{-}}{\partial v}=0
\end{aligned}
$$

and

$$
\frac{\mathrm{d}^{2} \phi}{\mathrm{d} \xi^{2}}=n^{+}-n^{-}
$$


The general solutions of Eqs. (54) and (55) are $f^{+}=f^{+}\left(\mathscr{E}^{+}\right)$and $f^{-}=f^{-}\left(\mathscr{E}^{-}\right)$, respectively, where the energy integrals $\mathscr{E}^{+}=\phi-\phi_{\max }+\mathscr{M}\left(v-u_{0}\right)^{2} / 2$ and $\mathscr{E}^{-}=\phi_{\min }-\phi+\left(v-u_{0}\right)^{2} / 2$, and where $\phi_{\min }$ and $\phi_{\max }$ is the minimum and maximum values of the potential, respectively. Here we study the existence of localized phase-space holes, where negatively charged particles are trapped in a localized potential hump. Therefore we assume that $\phi_{\min }=0$ at $|\xi|=\infty$, and omit the symbol $\phi_{\min }$ below. (An analogous case is phase-space holes in which positive ions are trapped in a negative potential well attributed by a localized potential minimum.) Positive values of $\mathscr{E}^{+}$and $\mathscr{E}^{-}$correspond to free particles, while negative values correspond to reflected or trapped particles. At the position where $\phi=\phi_{\max }$, there exist only free positively charged ions, and following Refs. [25,20], we set at this point a Maxwellian particle distribution for the positive ions, while at $|\xi|=\infty$ where $\phi=0$, we assume a Maxwellian distribution for the negative ions. This gives the free particle distribution for the positive ions

$$
f_{\mathrm{f}}^{+}\left(\mathscr{E}^{+}\right)= \begin{cases}a^{+}\left(\frac{\mathscr{M}}{2 \pi \mathscr{T}}\right)^{1 / 2} \exp \left[-\frac{\mathscr{M} \tilde{v}_{+}^{+}\left(\mathscr{E}^{+}\right)^{2}}{2 \mathscr{T}}\right], & v>v_{+}^{+}, \\ a^{+}\left(\frac{\mathscr{M}}{2 \pi \mathscr{T}}\right)^{1 / 2} \exp \left[-\frac{\mathscr{M} \tilde{v}_{-}^{+}\left(\mathscr{E}^{+}\right)^{2}}{2 \mathscr{T}}\right], & v<v_{-}^{+},\end{cases}
$$

where $\mathscr{T}=T^{+} / T^{-}, T^{+}$is the temperature of the positive ions, $\tilde{v}_{ \pm}^{+}\left(\mathscr{E}^{+}\right)=u_{0} \pm\left(2 \mathscr{E}^{+} / \mathscr{M}\right)^{1 / 2}$, and $v_{ \pm}^{+}=u_{0} \pm$ $(2 / \mathscr{M})^{1 / 2}\left(\phi_{\max }-\phi\right)^{1 / 2}$. For the negative ions we have

$$
f_{\mathrm{f}}^{-}\left(\mathscr{E}^{-}\right)= \begin{cases}(2 \pi)^{-1 / 2} \exp \left[-\frac{\tilde{v}_{+}^{-}\left(\mathscr{E}^{-}\right)^{2}}{2}\right], & v>v_{+}^{-}, \\ (2 \pi)^{-1 / 2} \exp \left[-\frac{\tilde{v}_{-}^{-}\left(\mathscr{E}^{-}\right)^{2}}{2}\right], & v<v_{-}^{-},\end{cases}
$$

where $\tilde{v}_{ \pm}^{-}\left(\mathscr{E}^{-}\right)=u_{0} \pm\left(2 \mathscr{E}^{-}\right)^{1 / 2}$ and $v_{ \pm}^{-}=u_{0} \pm(2 \phi)^{1 / 2}$. The constant $a^{+}$(to be specified later) normalizes the total positive ion particle density to unity at $|\xi|=\infty$ where $\phi=0$. On each side of the potential maximum there are populations of positive ions which are reflected, and for which we assume a flat particle distribution, $f^{+}=f_{r}^{+}=$constant. Such flat parts of the distribution function is a natural product of quasi-linear diffusion processes in plasmas, or of colliding potential structures. The value of the flat distribution function should be equal to the one of the free positive ion particle distribution function at the separatrices at $\phi=\phi_{\max }$. This gives

$$
f_{\mathrm{r}}^{+}=a^{+}\left(\frac{\mathscr{M}}{2 \pi \mathscr{T}}\right)^{1 / 2} \exp \left(-\frac{\mathscr{M} u_{0}^{2}}{2 \mathscr{T}}\right), \quad v_{-}^{+} \leqslant v \leqslant v_{+}^{+} .
$$

For the trapped negative ions, we choose a vortex solution of the form

$$
f_{\mathrm{t}}^{-}=\frac{1}{(2 \pi)^{1 / 2}} \exp \left[-\frac{u_{0}^{2}}{2}-\beta^{-} \mathscr{E}^{-}\right], \quad v_{-}^{-} \leqslant v \leqslant v_{+}^{-},
$$

where $\beta^{-}$is the trapping parameter describing the "temperature" of the trapped negative ions.

By integrating the particle distribution functions of the free particle species over velocity space, we obtain the densities of the free positive and negative ions as $[25,20]$

$$
n_{\mathrm{f}}^{+}=a^{+} \exp \left(-\frac{\mathscr{M} u_{0}^{2}}{2 \mathscr{T}}\right)\left[I\left(\frac{\phi_{\max }-\phi}{\mathscr{T}}\right)+\kappa\left(\frac{\mathscr{M} u_{0}^{2}}{2 \mathscr{T}}, \frac{\phi_{\max }-\phi}{\mathscr{T}}\right)\right]
$$

and

$$
n_{\mathrm{f}}^{-}=\exp \left(-\frac{u_{0}^{2}}{2}\right)\left[I(\phi)+\kappa\left(\frac{u_{0}^{2}}{2}, \phi\right)\right],
$$

respectively. The special functions $I$ and $\kappa$ are given in Eqs. (11) and (12), respectively. 

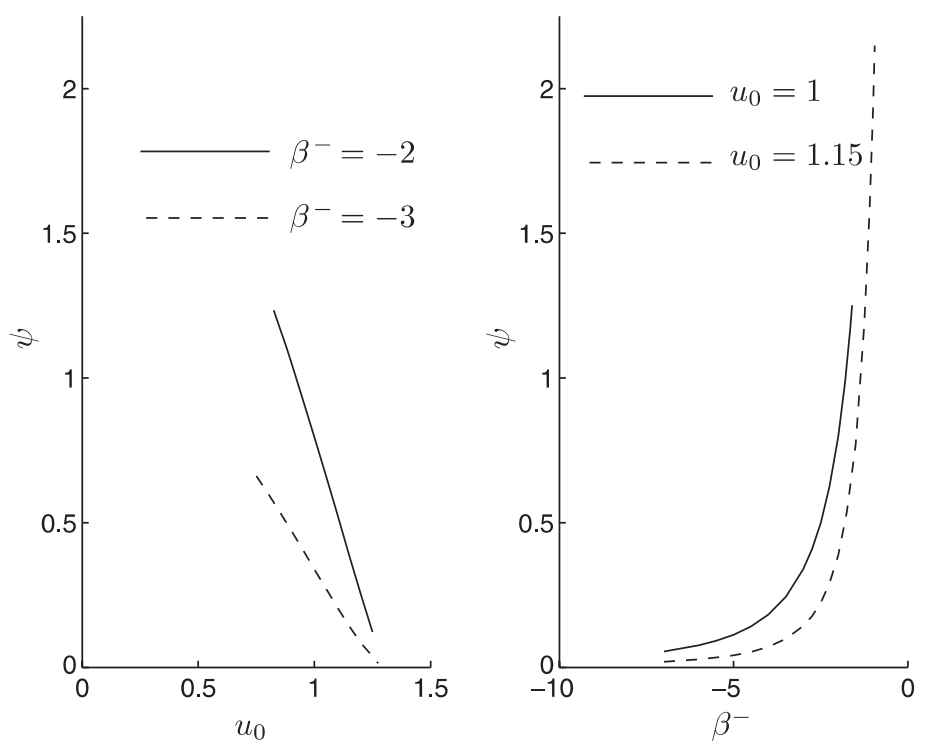

Fig. 19. The amplitude $\psi=\phi_{\max }$ of the potential for a phase-space hole of negative ions, as a function of the speed $u_{0}$ of the hole (left panel), with $\beta^{-}=-2$ (solid line) and $\beta^{-}=-3$ (dashed line), and the amplitude as a function of $\beta^{-}$(right panel), with $u_{0}=1.0$ (solid line) and $u_{0}=1.15$ (dashed line). We used $\mathscr{T}=\mathscr{M}=1$ for all cases (after Ref. [122]).
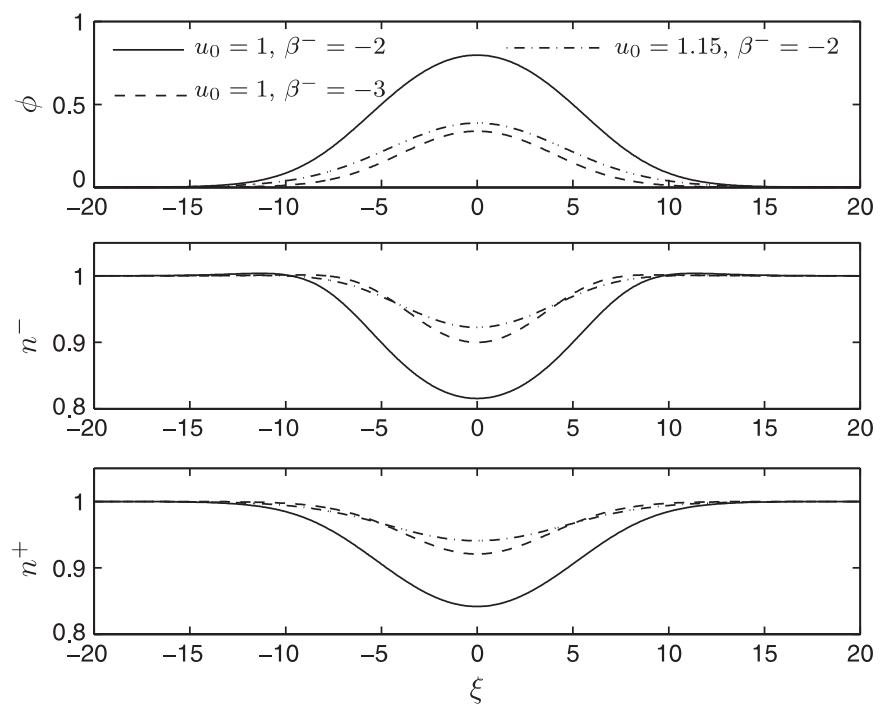

Fig. 20. The phase-space hole potential (upper panel), the density of the negatively charged ions (middle panel) and of the positive ions (lower panel) as a function of $\xi$, for $u_{0}=1$ and $\beta^{-}=-2$ (solid lines), for $u_{0}=1$ and $\beta^{-}=-3$ (dashed lines) and for $u_{0}=1.15$ and $\beta^{-}=-2$ (dash-dotted lines). We used $\mathscr{T}=\mathscr{M}=1$ for all cases (after Ref. [122]).

The particle density of the reflected positive ions is obtained by integrating the distribution function of the reflected ions over velocity space. We have

$$
\begin{aligned}
n_{\mathrm{r}}^{+} & =\int_{v_{i-}}^{v_{i+}} f_{\mathrm{r}}^{+} \mathrm{d} v=\left(v_{i+}-v_{i-}\right) f_{\mathrm{r}}^{+} \\
& =\frac{2 a^{+}}{(\pi \mathscr{T})^{1 / 2}}\left(\phi_{\max }-\phi\right)^{1 / 2} \exp \left(-\frac{\mathscr{M} u_{0}^{2}}{2 \mathscr{T}}\right) .
\end{aligned}
$$



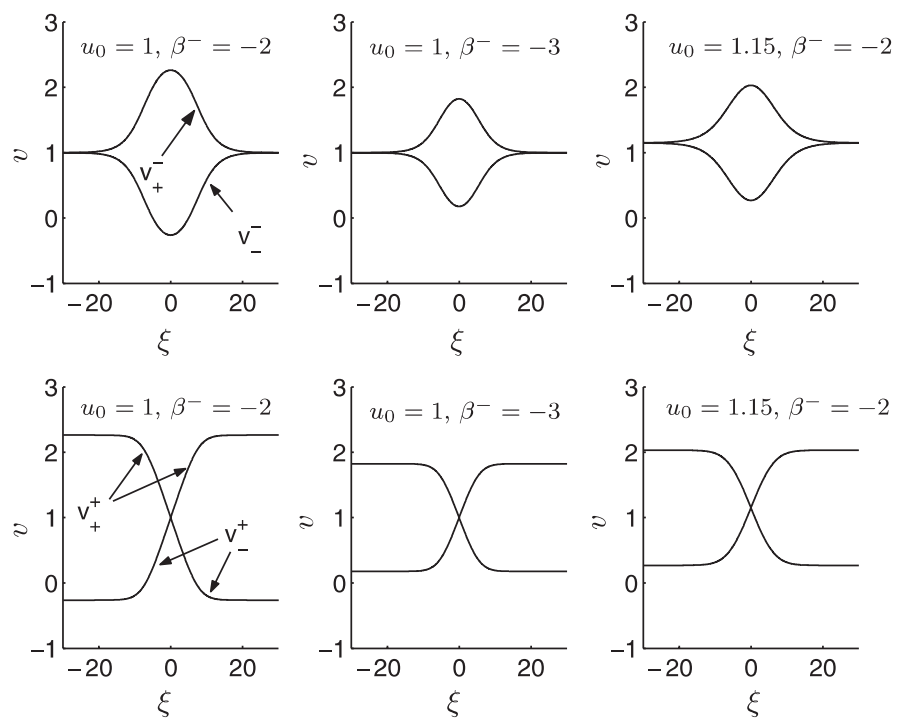

Fig. 21. The separatrices between free and trapped negatively charged ions in phase $(\xi, v)$ space (upper panels) and the separatrices between free and reflected positively charged ions (lower panels), for $u_{0}=1$ and $\beta^{-}=-2$ (left panels), for $u_{0}=1$ and $\beta^{-}=-3$ (middle panels) and for $u_{0}=1.15$ and $\beta^{-}=-2$ (right panels). We used $\mathscr{T}=\mathscr{M}=1$ for all cases (after Ref. [122]).
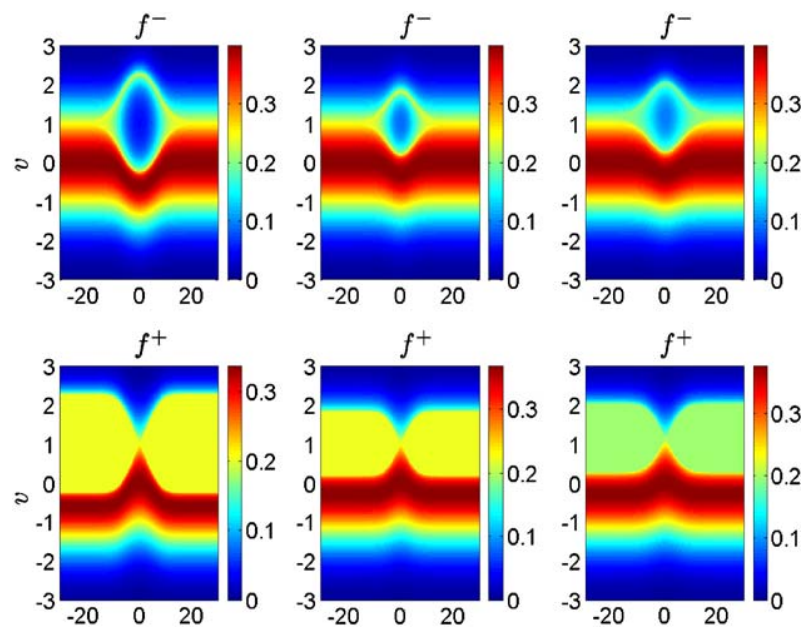

Fig. 22. The particle distribution function for negatively charged ions (upper panels) and for positively charged ions (lower panels) in phase ( $\xi$, $v$ ) space, for $u_{0}=1$ and $\beta^{-}=-2$ (left panels), for $u_{0}=1$ and $\beta^{-}=-3$ (middle panels) and for $u_{0}=1.15$ and $\beta^{-}=-2$ (right panels). We used $\mathscr{T}=\mathscr{M}=1$ for all cases (after Ref. [122]).

The number density of the trapped negative ions is

$$
n_{\mathrm{t}}^{-}=\frac{2}{\left(\pi\left|\beta^{-}\right|\right)^{1 / 2}} \exp \left(-\frac{u_{0}^{2}}{2}\right) W_{\mathrm{D}}\left[\left(-\beta^{-} \phi\right)^{1 / 2}\right] .
$$

The total positive ion number density is normalized to unity where $\phi=0$. Consequently, the condition that the total ion density is $n^{+}=n_{\mathrm{r}}^{+}+n_{\mathrm{f}}^{+}=1$ where $\phi=0$, gives the normalization constant

$$
a^{+}=\exp \left(\frac{\mathscr{M} u_{0}^{2}}{2 \mathscr{T}}\right)\left[\frac{2 \phi_{\max }^{1 / 2}}{(\pi \mathscr{T})^{1 / 2}}+I\left(\frac{\phi_{\max }}{\mathscr{T}}\right)+\kappa\left(\frac{\mathscr{M} u_{0}^{2}}{2 \mathscr{T}}, \frac{\phi_{\max }}{\mathscr{T}}\right)\right]^{-1} .
$$



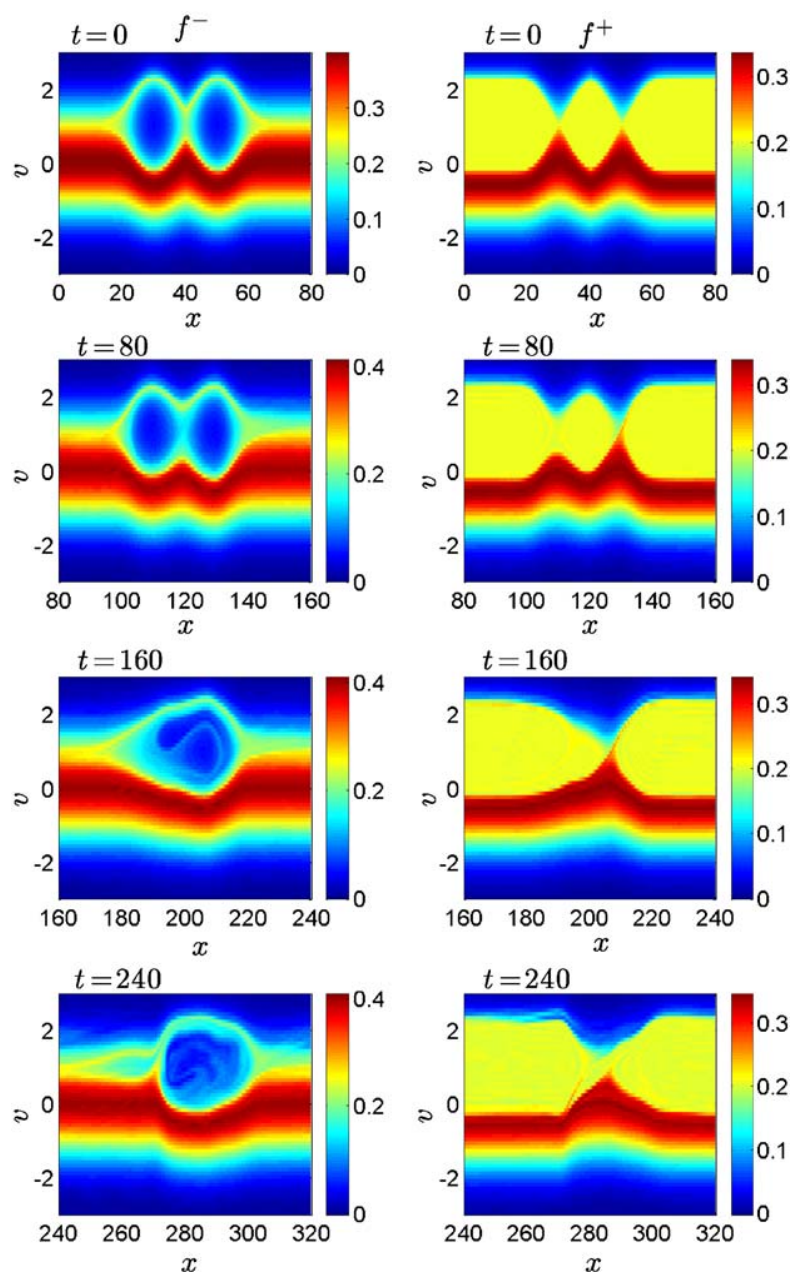

Fig. 23. The particle distribution function for negatively charged ions (left panels) and for positively charged ions (right panels) in phase ( $x, v)$ space for interacting phase-space holes, at different times (after Ref. [122]).

The total particle densities $n^{-}=n_{\mathrm{f}}^{-}+n_{\mathrm{t}}^{-}$and $n^{+}=n_{\mathrm{f}}^{+}+n_{\mathrm{r}}^{+}$inserted into Poisson's equation (56) gives an equation for the self-consistent electrostatic potential.

Figs. 19-22 display numerical solutions of Eq. (56) with the particle densities given by Eqs. (61)-(64). We have here used $\mathscr{T}=\mathscr{M}=1$, which is typical for an isothermal pair-ion plasma. Fig. 19 shows how the amplitude of the potential depends on $u_{0}$ and $\beta^{-}$. We see that phase-space holes typically have speeds close to the ion thermal speed, so that $u_{0} \approx 1$. The amplitude of the phase-space hole potential decreases with increasing speeds and vanishes for $u_{0}$ larger than approximately 1.3. The hole potential decreases with larger negative values of $\beta^{-}$. The profiles of the potential and particle densities are shown in Fig. 20 for typical values of $u_{0}$ and $\beta^{-}$. We see that the width of the localized pulses is of the order of 20 ion Debye radii, and that the phase-space hole is characterized by a correlated depletion of the densities of both the positive and negative ions. To compare with the experimental conditions [123] of a fullerene plasma with the temperatures $1.5 \mathrm{eV}$, the values of $\psi$ and $\phi$ in Figs. 19 and 20 should be multiplied by the factor 1.5 to obtain the corresponding values of $\sim 1-3 \mathrm{~V}$, and we note that the pulse width corresponds to $\sim 4 \mathrm{~cm}$ in the experiment where the ion Debye radius was $\sim 0.2 \mathrm{~cm}$. In Fig. 21 , we show the separatrix in $(\xi, v)$ space between the free particles and the trapped negative ions and reflected positive ions for the same set of parameters as in Fig. 20. The corresponding phase-space densities of the particle species are shown in Fig. 22. We see the trapped particle distribution, which is characterized by a local depletion in phase-space in the upper panels of Fig. 22, while the reflected, flat particle distribution is clearly seen in the lower panels of Fig. 22. 
In order to investigate the stability and dynamics of large-amplitude phase-space holes, we have solved the original Eqs. (51)-(54) with initial conditions corresponding to the case $u_{0}=1$ and $\beta^{-}=-2$ in Figs. 19-22. In Fig. 23, we observe nonlinear interactions between two large amplitude phase-space holes, which were initially placed near each other. The two propagating phase-space holes merge and form one single phase-space hole, which survives till the end of the simulation. It, therefore, seems that phase-space holes in pair-ion plasmas can be long-lived, and nonlinearly interacting holes tend to collide inelastically and merge into a new hole, similar to an electron hole in an electron-ion plasma. The numerical code used to produce Fig. 23 is based on a Fourier transform method in velocity space [109].

We suggest that future experiments should consider the possibility of observing our proposed new ion hole structures, similar to what has been done by Oohara et al. [124,125] for studying experimentally the dynamical evolution of plasma structures (DL-induced solitary pulses) due to local production of massive ions. Our results are also relevant for understanding the salient features of coherent nonlinear structures and their dynamics in both electron-positron plasmas [114,80], as well as for plasmas in which positively and negatively charged dust grains co-exist [126-128].

\section{Relativistic effects on phase-space holes}

So far we have discussed the properties and dynamics of non-relativistic electron and ion holes in electron-ion and pair-ion unmagnetized plasmas. In this section, we extend the electron hole theory to a situation where relativistic electron mass variation is significant due to extremely large amplitudes of holes in relativistically hot plasmas. The latter are quite common in astrophysical settings and also in the present generation laser-plasma interaction experiments devoted to inertially confinement fusion and modelling astrophysical situations in Laboratory experiments on Earth.

\subsection{Properties of relativistic electron holes}

Eliasson and Shukla [129] have developed a theory for the steady state relativistic electron holes (REHs) in a relativistically hot plasma [129]. They find that the properties of the electron holes are dramatically changed by the relativistic mass increase of the electrons. Their relativistic simulation studies show that strongly relativistic electron holes are long-lived, and that they survive head-on collisions. We expect that intense electron hole potentials can accelerate electrons to extremely high energies.

The dimensionless relativistic Vlasov-Poisson system, where ions are assumed to form a neutralizing background, is

$$
\frac{\partial f_{\mathrm{e}}}{\partial t}+\frac{p}{\sqrt{1+\mu^{2} p^{2}}} \frac{\partial f_{\mathrm{e}}}{\partial x}+\frac{\partial \phi_{\mathrm{e}}}{\partial x} \frac{\partial f_{\mathrm{e}}}{\partial p}=0
$$

and

$$
\frac{\partial^{2} \phi_{\mathrm{e}}}{\partial x^{2}}=\int_{-\infty}^{\infty} f_{\mathrm{e}} \mathrm{d} p-1,
$$

where $\mu=V_{T e} / c$, the distribution function $f_{\mathrm{e}}$ has been normalized by $n_{0} / m_{\mathrm{e}} V_{T e}, t$ by $\omega_{\mathrm{pe}}^{-1}, x$ by $r_{\mathrm{De}}$, the momentum $p$ by $m_{\mathrm{e}} V_{T e}$, and $\phi_{\mathrm{e}}$ by $k_{\mathrm{B}} T_{\mathrm{e}} / e$. Here, $c$ is the speed of light in vacuum and the non-relativistic plasma frequency is $\omega_{\mathrm{pe}}=\left(4 \pi n_{0} e^{2} / m_{\mathrm{e}}\right)^{1 / 2}$. We (the observer) remain in the frame of the bulk plasma while the electron hole is moving with the speed $u_{0}$. Accordingly, we look for solutions of the form $f(p, \xi)$ and $\phi_{\mathrm{e}}(\xi)$, where $\xi=x-M_{\mathrm{e}} t, M_{\mathrm{e}}=u_{0} / V_{T e}$ is the Mach number and $u_{0}$ is the constant propagation speed of the electron hole. With this ansatz, the Vlasov-Poisson system (66) and (67) takes the form

$$
\left(-M_{\mathrm{e}}+\frac{p}{\sqrt{1+\mu^{2} p^{2}}}\right) \frac{\partial f_{\mathrm{e}}}{\partial \xi}+\frac{\mathrm{d} \phi_{\mathrm{e}}}{\mathrm{d} \xi} \frac{\partial f_{\mathrm{e}}}{\partial p}=0
$$

and

$$
\frac{\mathrm{d}^{2} \phi_{\mathrm{e}}}{\mathrm{d} \xi^{2}}=\int_{-\infty}^{\infty} f_{\mathrm{e}} \mathrm{d} p-1
$$


Integrating Eq. (68) along its trajectories, one finds the general solution of the form $f_{\mathrm{e}}=f_{0}(\mathscr{E})$, where $f_{0}$ is some function of one variable and

$$
\mathscr{E}=-M_{\mathrm{e}} p+\frac{1}{\mu^{2}}\left(\sqrt{1+\mu^{2} p^{2}}-\frac{1}{\gamma_{0}}\right)-\phi_{\mathrm{e}}
$$

is a conserved quantity (energy) along the particle trajectories. Here, we have denoted $\gamma_{0}=1 / \sqrt{1-\mu_{0}^{2} M_{\mathrm{e}}^{2}}$. At the electron hole, a population of the electrons is trapped in a localized positive potential $\phi_{\mathrm{e}}$, which goes to zero far away from the electron hole. The energy $\mathscr{E}$ has been shifted so that the trapped electrons have a negative energy, while the untrapped (free) electrons have a positive energy.

A condition for the untrapped electrons is that far away from the electron hole, the distribution should connect smoothly to a Jüttner-Synge distribution [130], which restricted to one momentum dimension takes the form

$$
\tilde{f}_{0}(p)=a_{0} \exp \left[-\frac{1}{\mu^{2}}\left(\sqrt{1+\mu^{2} p^{2}}-1\right)\right]
$$

where the normalization constant is

$$
a_{0}=\left\{\int_{-\infty}^{\infty} \exp \left[-\frac{1}{\mu^{2}}\left(\sqrt{1+\mu^{2} p^{2}}-1\right)\right] \mathrm{d} p\right\}^{-1}=\frac{\mu \exp \left(-\mu^{-2}\right)}{2 K_{1}\left(\mu^{-2}\right)},
$$

and $K_{1}$ is a modified Bessel function of second kind. The weakly relativistic limit corresponds to $\mu \ll 1$ so that $a_{0} \approx\left(1+3 \mu^{2} / 8\right)^{-1} / \sqrt{2 \pi}$ [131], and Eq. (71) converges to a non-relativistic Maxwellian distribution when $\mu \rightarrow 0$. In order to impose the above-mentioned condition for the free electrons, we use the solution

$$
f_{\mathrm{e}}=f_{0}(\mathscr{E})=\tilde{f}_{0}[\tilde{p}(\mathscr{E})], \quad \mathscr{E}>0,
$$

where $\tilde{p}(\mathscr{E})$ is a function of the energy such that $\tilde{p}(\mathscr{E}) \rightarrow p$ when $\phi_{\mathrm{e}} \rightarrow 0$. Such a function can be found with the help of Eq. (70) by setting

$$
-M_{\mathrm{e}} \tilde{p}(\mathscr{E})+\frac{1}{\mu^{2}}\left(\sqrt{1+\mu^{2} \tilde{p}^{2}(\mathscr{E})}-\frac{1}{\gamma_{0}}\right)=\mathscr{E} .
$$

Solving for $\tilde{p}(\mathscr{E})$, we have

$$
\tilde{p}_{ \pm}(\mathscr{E})=\gamma_{0}^{2} M_{\mathrm{e}}\left(\mu^{2} \mathscr{E}+\frac{1}{\gamma_{0}}\right) \pm \frac{\gamma_{0}^{2}}{\mu} \sqrt{\left(\mu^{2} \mathscr{E}+\frac{1}{\gamma_{0}}\right)^{2}-\frac{1}{\gamma_{0}^{2}}},
$$

where $\tilde{p}_{+}(\mathscr{E})$ and $\tilde{p}_{-}(\mathscr{E})$ correspond to a modified momentum for free electrons on each side of the trapped electron population in momentum space. Using $\tilde{p}(\mathscr{E})=\tilde{p}_{+}(\mathscr{E})$ and $\tilde{p}(\mathscr{E})=\tilde{p}_{-}(\mathscr{E})$ in Eq. (73), we obtain the distribution function for the free electrons. In the limit of vanishing energy, $\mathscr{E}=0^{+}$, we have $\tilde{p}_{ \pm}(0)=\gamma_{0} M_{\mathrm{e}}$, and the value of the distribution function is

$$
\left.f_{\mathrm{e}}\right|_{\mathscr{E}=0^{+}}=f_{0}(0)=\tilde{f}_{0}\left(\gamma_{0} M_{\mathrm{e}}\right)=a_{0} \exp \left(-\frac{\gamma_{0}-1}{\mu^{2}}\right),
$$

which should be matched with the distribution function for the trapped electrons with $\mathscr{E}=0$ in order to obtain a continuous distribution function. For the trapped electrons, we choose a relativistic Maxwell-Boltzmann distribution with a negative "temperature", viz.

$$
f_{\mathrm{e}}=a_{0} \exp \left(-\frac{\gamma_{0}-1}{\mu^{2}}-\beta_{\mathrm{e}} \mathscr{E}\right), \quad \mathscr{E}<0,
$$

leading to a vortex distribution for $\beta_{\mathrm{e}}<0$, a flat top distribution for $\beta_{\mathrm{e}}=0$ and a shifted Jüttner-Synge distribution for $\beta_{\mathrm{e}}=1$. The separatrix between the free and trapped electron distributions is found where $\mathscr{E}=0$ in Eq. (70). Solving for $p$ in Eq. (70) with $\mathscr{E}=0$, we have

$$
p_{ \pm}=\gamma_{0}^{2} M_{\mathrm{e}}\left(\mu^{2} \phi_{\mathrm{e}}+\frac{1}{\gamma_{0}}\right) \pm \frac{\gamma_{0}^{2}}{\mu} \sqrt{\left(\mu^{2} \phi_{\mathrm{e}}+\frac{1}{\gamma_{0}}\right)^{2}-\frac{1}{\gamma_{0}^{2}}},
$$




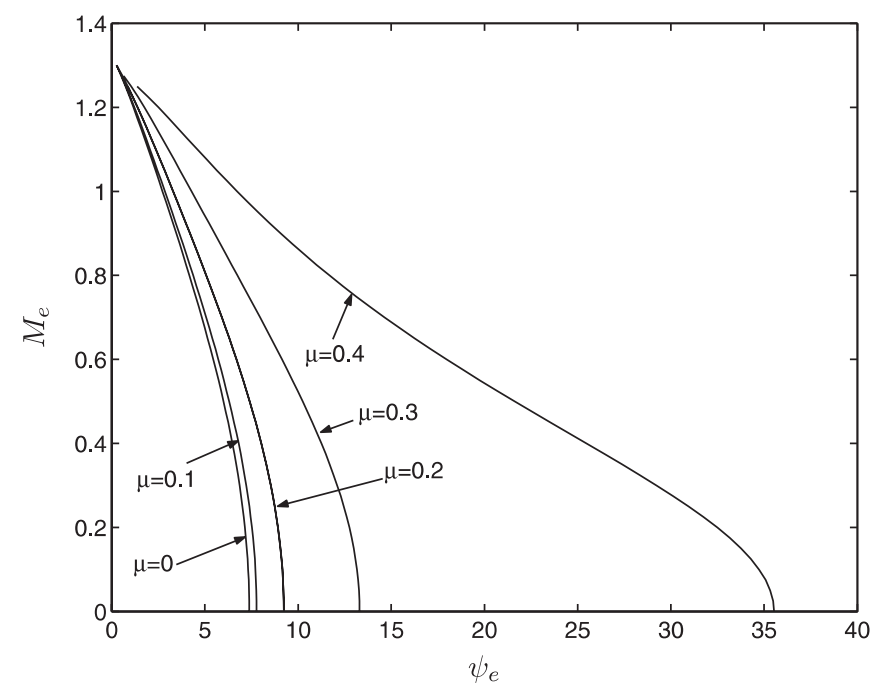

Fig. 24. The Mach number $M_{\mathrm{e}}$ of the electron hole as a function of the maximum amplitude $\psi$ of the electron hole potential $\phi_{\mathrm{e}}$, for a constant trapping parameter $\beta_{\mathrm{e}}=-0.5$ and for different values of $\mu$ (after Ref. [129]).

where $p_{-}\left(\phi_{\mathrm{e}}\right)$ and $p_{+}\left(\phi_{\mathrm{e}}\right)$ constitute the limits between the trapped and free electron distributions in momentum space. Using these limits and combining Eqs. (73), (75) and (77), we can now write the full electron distribution function as

$$
f_{\mathrm{e}}= \begin{cases}a_{0} \exp \left[-\frac{1}{\mu^{2}}\left(\sqrt{1+\mu^{2} \tilde{p}_{+}^{2}(\mathscr{E})}-1\right)\right], & p>p_{+}, \\ a_{0} \exp \left[-\frac{\gamma_{0}-1}{\mu^{2}}-\beta_{\mathrm{e}} \mathscr{E}\right], & p_{-} \leqslant p \leqslant p_{+}, \\ a_{0} \exp \left[-\frac{1}{\mu^{2}}\left(\sqrt{1+\mu^{2} \tilde{p}_{-}^{2}(\mathscr{E})}-1\right)\right], & p<p_{-},\end{cases}
$$

where $\mathscr{E}$ is given by Eq. (70). Integrating the distribution function over momentum space we obtain the total electron density as a function of $\phi_{\mathrm{e}}$, which is calculated self-consistently by means of Poisson's equation (69).

In order to proceed further and to explore the impact of the relativistic effects on the electron holes for different sets of parameters, we have solved Poisson's equation (69) numerically, where the integration of the trapped and free electron populations has been done with a sum representation of the integrals, and Eq. (69) has been solved as a nonlinear boundary value problem where the potential has been set to zero far away from the electron hole, and the resulting nonlinear system of equations have been solved iteratively with a Newton-like method. An alternative would be to use the so-called potential method [22]. In Fig. 24 we display the electron hole Mach number $M_{\mathrm{e}}$ as a function of the maximum amplitude $\psi$ of the potential for different values of the parameter $\mu$. We see that for larger values of $\mu$, the amplitude of the electron holes drastically increases. Note that the electron hole potential has been normalized by $k_{\mathrm{B}} T_{\mathrm{e}} / e$, so the curves in Fig. 24 show pure relativistic effects due to the relativistic mass increase of the electrons. In the non-relativistic limit $(\mu=0)$, we recover the previous results of Bujarbarua and Schamel [26]; see their Fig. 1.

The profiles of the electrostatic potential and the electron density are shown in Fig. 25 for different sets of parameters. Comparing cases (a) and (b) of Fig. 25, we see a dramatic difference in the size of the electron hole. In case (a), the temperature is twice as high as in case (b), which results in a much larger potential and width of the electron hole. To convert the potential to volts, the values of $\phi_{\mathrm{e}}$ should be multiplied by the factor $5.1 \times 10^{5} \mu^{2}$; accordingly, the amplitude of the potential for case (a) $(\psi \approx 33, \mu=0.4)$ is $\approx 2.4 \times 10^{6} \mathrm{~V}$, which can accelerate electrons to strongly relativistic energies, while for case (b) $(\psi \approx 9, \mu=0.2)$ it is $\approx 0.18 \times 10^{6} \mathrm{~V}$. Cases (c) and (d), which should be compared with case (a), shows that the REH becomes smaller when its velocity increases and when the trapping parameter $\beta_{\mathrm{e}}$ has larger negative values, similar as for non-relativistic electron holes [26]. The electron distribution of the electron holes in phase space is shown in Fig. 26 for the same sets of parameters as in Fig. 25. We see that the electrons are accelerated to strongly relativistic energies, especially in case (a) $(\mu=0.4)$ where the fastest electrons with momentum $p \approx 15$ have a gamma factor of $\gamma=\sqrt{1+\mu^{2} p^{2}} \approx 6$. 

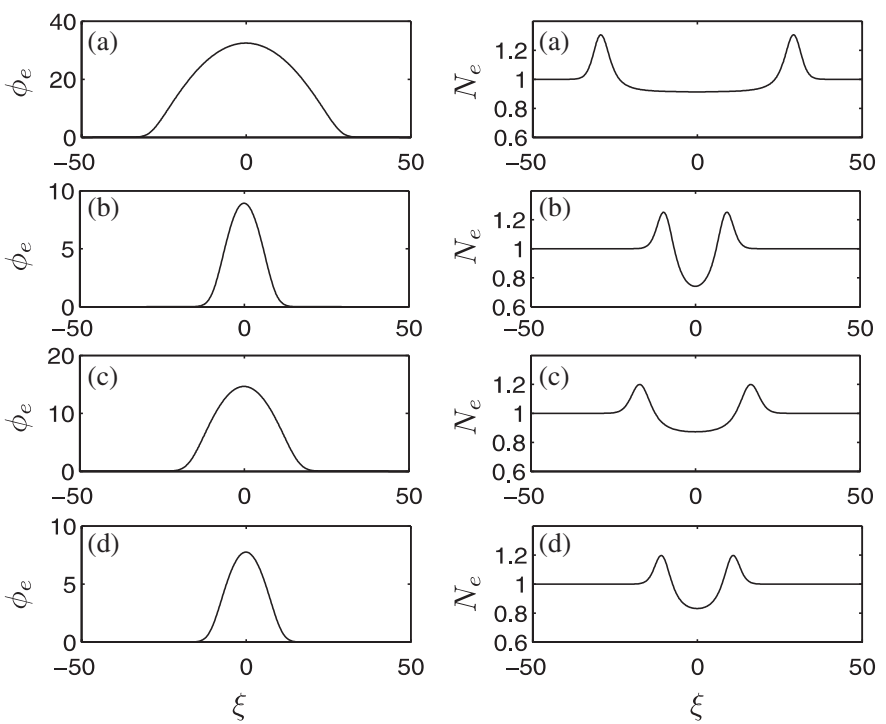

Fig. 25. The potential (left panel) and electron density (right panel) of electron holes with (a) $\beta_{\mathrm{e}}=-0.5, \mu=0.4$ and $M_{\mathrm{e}}=0.2$; (b) $\beta_{\mathrm{e}}=-0.5$, $\mu=0.2$ and $M_{\mathrm{e}}=0.2 ;$ (c) $\beta_{\mathrm{e}}=-0.5, \mu=0.4$ and $M_{\mathrm{e}}=0.7$; and (d) $\beta_{\mathrm{e}}=-0.7, \mu=0.4$ and $M_{\mathrm{e}}=0.2$ (after Ref. [129]).
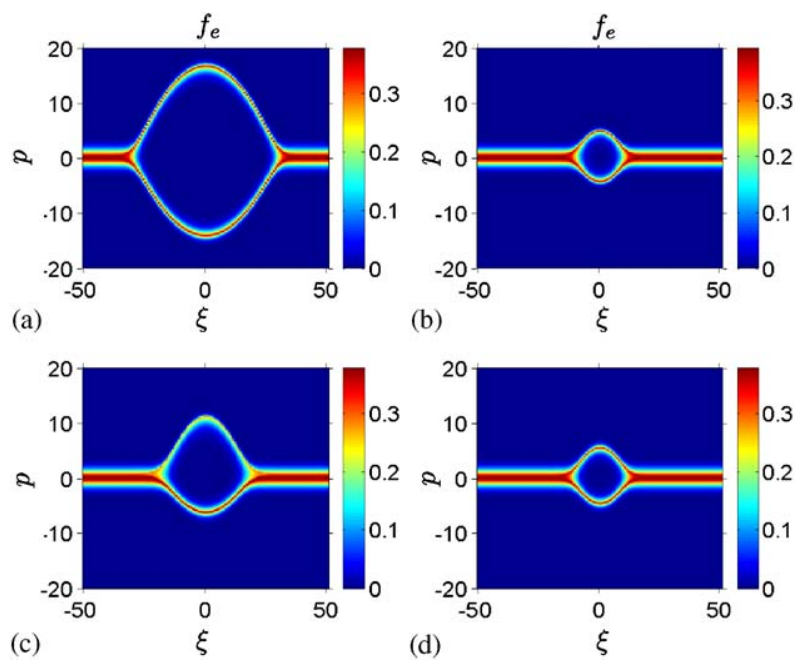

Fig. 26. Phase-space plots for electron holes with: (a) $\beta_{\mathrm{e}}=-0.5, \mu=0.4$ and $M_{\mathrm{e}}=0.2$; (b) $\beta_{\mathrm{e}}=-0.5, \mu=0.2$ and $M_{\mathrm{e}}=0.2$; (c) $\beta_{\mathrm{e}}=-0.5$, $\mu=0.4$ and $M_{\mathrm{e}}=0.7$; and (d) $\beta_{\mathrm{e}}=-0.7, \mu=0.4$ and $M_{\mathrm{e}}=0.2$, corresponding to the cases (a)-(d) in Fig. 25 (after Ref. [129]).

In order to investigate the time-dependent dynamics of REHs, we have solved the Vlasov-Poisson system (66)-(67) numerically. The results are shown in Fig. 27. The left hole has initially the Mach number $M_{\mathrm{e}}=0.7$ and the right hole has a negative Mach number $M_{\mathrm{e}}=-0.2$, while both holes have $\beta_{\mathrm{e}}=-0.5$ and $\mu=0.4$. During head-on collisions, the two holes merge and form a new electron hole, which survives through the rest of the simulation time. As a comparison, shown in Fig. 28, we changed the relativistic factor to $\mu=0.01$, while keeping the same trapping parameter $\beta_{\mathrm{e}}=-0.5$ and the initial speeds of the holes. In this case, the electron holes are considerably smaller, and do not merge as they did in the strongly relativistic case. The numerical method used in our simulations was a pseudo-spectral method to approximate the $x$-derivatives, a fourth-order compact Padé scheme to approximate the $p$ derivative [132], and the fourth-order Runge-Kutta method for the time stepping. The integral over $p$ space in Eq. (67) was done with a simple sum representation of the integral. 

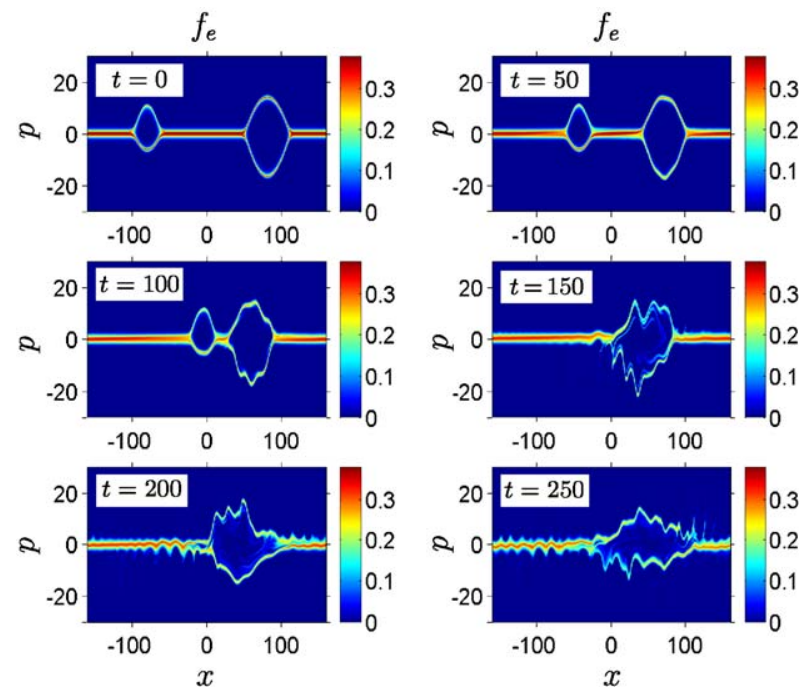

Fig. 27. Interactions between strongly relativistic electron holes. Initially, the parameters are $\mu=0.4$ and $\beta_{\mathrm{e}}=-0.5$ for both holes, and the left and right holes have the Mach numbers $M_{\mathrm{e}}=0.7$ and -0.2 , respectively, corresponding to cases (a) and (c) in Figs. 25 and 26. Time intervals are: $t=0$ (upper left panel), $t=50$ (upper right panel), $t=100$ (middle left panel), $t=150$ (middle right panel), $t=200$ (lower left panel), and $t=250$ (lower right panel) (after Ref. [129]).
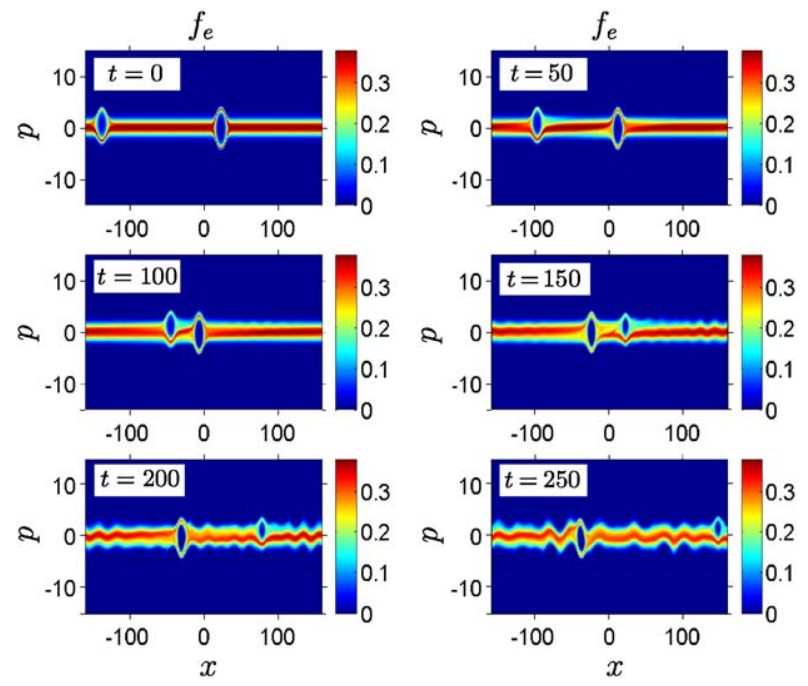

Fig. 28. Interactions between weakly relativistic electron holes. Initially, the parameters are $\mu=0.01$ and $\beta_{\mathrm{e}}=-0.5$ for both holes, and the left and right holes have the Mach number $M_{\mathrm{e}}=0.7$ and -0.2 , respectively. Times: $t=0$ (upper left panel), $t=50$ (upper right panel), $t=100$ (middle left panel), $t=150$ (middle right panel), $t=200$ (lower left panel) and $t=250$ (lower right panel) (after Ref. [129]).

\section{Magnetized plasmas}

Laboratory and space plasmas are often embedded in an external magnetic field. The latter introduces anisotropy in the system. The particle trapping in an external magnetic field is quite complex, leave alone the coherent nonlinear structures that we have discussed so far. In this section, we present some flavor of the trapped particle distribution functions that are affected by the external magnetic field, as well as discuss possible electron and ion holes that are 
asymmetric. The results have relevance to the understanding of nonlinear coherent structures associated with phasespace vortices in the Earth's ionospheric and magnetospheric plasmas.

\subsection{Drift-kinetic theory of electron holes}

We formulate a drift-kinetic theory for electron phase-space vortices in magnetized plasmas [133]. It provides a model for the spatial and temporal scales of asymmetric electrostatic bipolar structures that have been observed by the GEOTAIL and FAST spacecrafts, in the Earth's magnetotail and in the auroral zone. For example, recent satellite observations from the FAST $[47,48]$ and POLAR spacecrafts $[53,58]$ revealed the signatures of electron holes in the transition layer between the Earth's ionosphere and the magnetosphere, and in the higher $(5000 \mathrm{~km})$ auroral region of the magnetosphere, respectively, as well as in the Earth's deep magnetotail by the GEOTAIL satellite [134]. Thus, the presence of 3D electron holes appears to be a universal feature of the near-Earth plasmas. Our nonlinear model accounts for the effects of both the electron polarization and the anisotropic electron temperature. Several different types of quasi-3D electron holes are found, in the form of either cylinders or ellipsoids. Although topologically different, they produce similar signals on the spacecraft under typical FAST and GEOTAIL observational conditions, and cannot be distinguished with the use of the existing scarce satellite observational data. The perpendicular velocities of the structures are substantially smaller than their parallel components. The recent fully 3D PIC computer simulations by Singh et al. [137], successfully reproduced the main properties of the FAST and POLAR electrostatic pulses, and showed that the full three-dimensionality is crucial for the rapid saturation of the oblique resonance cone mode (also called the Fisher-Gould mode), as well as for the long-time stability of the resulting 3D electron holes. However, in those simulations, the role of the $\mathbf{E} \times \mathbf{B}$ plasma motion, and of the possible coupling to other electrostatic modes, such as the upper-hybrid waves, were not addressed.

In the following, we discuss the properties of electron holes in the frequency range of electron whistler waves, i.e. $\omega_{\mathrm{ci}} \ll|\mathrm{d} / \mathrm{d} t| \ll \omega_{\mathrm{ce}}$, where $\omega_{\mathrm{ce}}=e B_{0} / m_{\mathrm{e}}$ is the electron gyrofrequency, in order to provide a theoretical explanation for the POLAR events discussed in Refs. [53] (their Fig. 1) and [58] and FAST events [47,48]. Here, $|\mathrm{d} / \mathrm{d} t|$ denotes the typical "frequency" of the electron hole, as recorded by satellites, and is defined as the speed of the hole relative the bulk plasma divided by its length scale. Those structures were observed in plasmas with highly anisotropic electron temperatures, $T_{\mathrm{e} \|} \gg T_{\mathrm{e} \perp}$, where the subscripts $\|$ and $\perp$ correspond to the directions parallel, and perpendicular to the magnetic field, respectively, which were streaming along the magnetic field lines with a velocity comparable to the parallel electron thermal speed $V_{T e \|}=\left(k_{\mathrm{B}} T_{\mathrm{e} \|} / m_{\mathrm{e}}\right)^{1 / 2}$. Their parallel spatial scale was several times larger than the electron Debye length $r_{\text {De } \|}=V_{T e \|} / \omega_{\text {pe }}$ and the perpendicular scale was somewhat larger than the parallel one. The velocity of the structures along the magnetic field was comparable to the parallel electron thermal speed $V_{T e \|}$, and consequently the characteristic frequency was between the electron and ion gyrofrequencies, while the perpendicular velocity was much smaller. The plasma of the auroral region of the magnetosphere can be regarded as strongly magnetized, with $\omega_{\text {ce }} / \omega_{\text {pe }} \sim 5$. Conversely, in the deep magnetotail we have $\omega_{\mathrm{ce}} / \omega_{\mathrm{pe}} \leqslant 1 / 5$.

Using a second-order drift-kinetic description of the electrons [140], that includes also the finite Larmor radius and polarization effects, we account for the electron kinetic effects associated with their motion along the magnetic field. In the frequency range of whistlers, regarding the ions as immobile, we find a fully nonlinear coherent electrostatic solution that can be interpreted as an electron hole coupled with a nonlinear upper-hybrid wave. The hole has either an ellipsoidal or cylindrical shape, the latter being slightly tilted relative to the magnetic field.

We restrict ourselves to the nonlinear modes whose parallel phase velocity and perpendicular characteristic wavelength scale as the parallel electron thermal velocity $V_{T e \|}$ and the Debye radius $r_{\text {De } \|}$, respectively. Then, in the nonrelativistic plasmas, $V_{T e \|} \ll c$, we may neglect the perturbation of the magnetic field and regard these modes as purely electrostatic. Since the parallel scale of these structures is of the order of $\sim 1 \mathrm{~km}$, we may also neglect the curvature and the inhomogeneity of the magnetic field.

The plasma is assumed to be weakly inhomogeneous with the unperturbed density given by $n^{(0)}(x)=n_{0}\left(1-x / L_{n}\right)$, which is immersed in an inhomogeneous magnetic field $\hat{\mathbf{z}} B$. The scale length $L_{n}$ is typically of the order $100-1000 \mathrm{~km}$ or for ionospheric and magnetospheric plasmas, which is much larger than the scale size of the electron hole. The term $x / L_{n}$ will therefore drop out from Poisson's equation but will enter into the expression for the electron diamagnetic velocity in the following section. The electron temperature is anisotropic, $T_{\mathrm{e} \|} \gg T_{\mathrm{e} \perp}$. In the regime when the magnetic field lines may be regarded as straight, the gyrokinetic equation [Eqs. (37) and (39) in Ref. [140]] has the particularly 
simple form

$$
\frac{\partial \bar{f}}{\partial t}+\mathbf{V} \cdot \nabla \bar{f}-(\nabla \cdot \mathbf{V}) v_{\perp}^{2} \frac{\partial \bar{f}}{\partial v_{\perp}^{2}}+\frac{e}{m_{\mathrm{e}}} \frac{\partial \varphi}{\partial z} \frac{\partial \bar{f}}{\partial v_{\|}}=0,
$$

where $\bar{f}$ is the gyro-angle-averaged electron distribution function. The guiding center velocity $\mathbf{V}$ is defined as

$$
\mathbf{V}=v_{\|} \hat{\mathbf{z}}+\mathbf{V}_{E}+\mathbf{V}_{\nabla B}+\mathbf{V}_{p}
$$

where the $\mathbf{E} \times \mathbf{B}, \nabla B$ and polarization drift velocities are given by $\mathbf{V}_{E}=(1 / B) \hat{\mathbf{z}} \times \nabla \varphi, \mathbf{V}_{\nabla B}=-\left(v_{\perp}^{2} / 2 \omega_{\text {ce }}\right) \hat{\mathbf{z}} \times \nabla \ln B$, and $\mathbf{V}_{p}=\left(1 / \omega_{\mathrm{ce}}\right)\left(\partial / \partial t+v_{\|} \partial / \partial z+\mathbf{V}_{E} \cdot \nabla\right)\left(\nabla_{\perp} \varphi / B\right)$, respectively.

The gyrokinetic equation (80) was derived in Ref. [140] with a high- $\beta^{*}$ ordering procedure (where the plasma-beta $\beta^{*}=2 \mu_{0} n_{0} k_{\mathrm{B}} T_{\mathrm{e}} / B^{2}$ is the ratio between the thermal and magnetic pressures). In the present context, such ordering corresponds to

$$
\begin{aligned}
\frac{L_{\perp}}{L_{\|}} \sim \frac{\omega_{\mathrm{pe}}}{\omega_{\mathrm{ce}}} \sim \frac{\rho_{\mathrm{Le}}}{r_{\mathrm{De}}} \sim 1, \\
\frac{\omega}{\omega_{\mathrm{ce}}} \sim \frac{\rho_{\mathrm{Le}}}{L_{\perp}} \sim \frac{\left|\mathbf{V}_{E}\right|}{V_{T e}} \sim \frac{E_{\|}}{E_{\perp}} \sim \epsilon,
\end{aligned}
$$

(see Eqs. (24) and (25) in Ref. [140]). In the above, $\omega$ is the characteristic frequency, $L_{\perp}$ and $L_{\|}$are the perpendicular and parallel length scales, while $\rho_{\mathrm{Le}}=V_{T e} / \omega_{\mathrm{ce}}$ is the electron Larmor radius, and $\epsilon \ll 1$ is a small parameter. Obviously, if $\epsilon$ is small enough, the equations in Ref. [140] are applicable both under the FAST and GEOTAIL conditions, discussed above. Note that the scaling (82) permits the presence of large zero-order perpendicular electric fields. Following Ref. [140], we may relax the last condition in Eq. (82), and use instead the alternative ordering $E_{\|} / E_{\perp} \sim 1$, which is, in the electrostatic case, also in agreement with the ordering for the length scales.

In plasmas with highly anisotropic electron temperatures, $T_{\mathrm{e} \|} \gg T_{\mathrm{e} \perp}$, we may neglect the kinetic effects associated with the perpendicular electron dynamics, and simplify Eq. (80) further by the integration over the magnitude of the perpendicular velocity, $v_{\perp}$. Thus, to the leading order in the small parameter $\epsilon$, and in the absence of strong perpendicular currents (i.e. of zero-order electric fields) in the unperturbed plasma state, we obtain the drift-kinetic equation

$$
\left(\frac{\partial}{\partial t}+v_{\|} \frac{\partial}{\partial z}+\mathbf{V}_{E} \cdot \nabla_{\perp}+\frac{e}{m_{\mathrm{e}}} \frac{\partial \varphi}{\partial z} \frac{\partial}{\partial v_{\|}}\right) \mathscr{F}+\mathscr{F} \nabla \cdot\left(\mathbf{V}_{E}+\mathbf{V}_{p}\right)=0
$$

Here $\mathscr{F}=\int \mathrm{d} v_{x} \mathrm{~d} v_{y} \bar{f}$ is the electron distribution function integrated over two velocity components that are perpendicular to the magnetic field. We note that the term $\nabla \cdot \mathbf{V}_{E}$ vanishes identically in Eq. (83).

For perturbations whose characteristic perpendicular wavelength is much smaller than the scale length of the magnetic field inhomogeneity, we can neglect the $\nabla B$ drift and rewrite the drift-kinetic equation (83) as a homogeneous equation of the first order

$$
\left(\frac{\partial}{\partial t}+v_{\|} \frac{\partial}{\partial z}+\mathbf{V}_{E} \cdot \nabla_{\perp}+\frac{e}{m_{\mathrm{e}}} \frac{\partial \varphi}{\partial z} \frac{\partial}{\partial v_{\|}}\right) g=0
$$

where $g=\mathscr{F} /\left[1-\nabla_{\perp}^{2} \varphi /\left(B_{0} \omega_{\mathrm{ce}}\right)\right]$ and $B_{0}$ is the homogeneous geomagnetic field strength.

In a reference frame moving with the velocity $u_{z}$ along the $z$-axis, substituting $z \rightarrow z-u_{z} t$, the drift-kinetic equation (84) can be conveniently rewritten in a symmetric form

$$
\frac{1}{e B_{0}}[W, g]_{x, y}+\frac{1}{m_{\mathrm{e}}}[W, g]_{z, v_{\|}}=\frac{\partial g}{\partial t},
$$

where the Poisson bracket is defined as

$$
[F, G]_{p, q}=\frac{\partial F}{\partial p} \frac{\partial G}{\partial q}-\frac{\partial F}{\partial q} \frac{\partial G}{\partial p}
$$


and the electron energy is

$$
W=-e \varphi+\frac{m_{\mathrm{e}}}{2}\left(v_{\|}-u_{z}\right)^{2} .
$$

We note that Eq. (85) is invariant with respect to rotation around the $x$ (or $y$ ) axis, i.e. to the following transformation:

$$
\begin{aligned}
& x^{\prime}=x \cos \theta-\frac{m_{\mathrm{e}}}{e B_{0}}\left(v_{\|}-u_{z}\right) \sin \theta, \\
& y^{\prime}=y \cos \theta+z \sin \theta \\
& z^{\prime}=z \cos \theta-y \sin \theta, \\
& v_{\|}^{\prime}-u_{z}=\left(v_{\|}-u_{z}\right) \cos \theta+\frac{e B_{0}}{m_{\mathrm{e}}} x \sin \theta .
\end{aligned}
$$

We assume that in the unperturbed state there exists an electron flow along the magnetic field lines, which gives rise to a beam-plasma instability [137]. Following the standard electron hole scenario, we do not attempt to study the full plasma dynamics. Instead, we seek a saturated state which is propagating along the magnetic field with the velocity $u_{z}$. In the stationary case, $\partial g / \partial t=0$, the characteristics of the drift-kinetic equation (85) are determined from

$$
\frac{\mathrm{d} x}{\partial W / \partial y}=-\frac{\mathrm{d} y}{\partial W / \partial x}=\frac{1}{\omega_{\mathrm{ce}}} \frac{\mathrm{d} z}{\partial W / \partial v_{\|}}=-\frac{1}{\omega_{\mathrm{ce}}} \frac{\mathrm{d} v_{\|}}{\partial W / \partial z}
$$

which yields the conservation of the energy, $W=$ constant. The other integrals of motion are virtually impossible to calculate in the general case, since the spatial dependence of the potential $\varphi$ is not known in advance (actually, it is to be determined self-consistently by using Poisson's equation and the solution of the drift-kinetic equation). As a consequence, Eq. (85) is analytically integrable only in certain special cases, in which the electrostatic potential $\varphi$ depends on a reduced number of spatial variables. Formally, it would be possible to describe also the fully 3D holes, based only on the energy conservation in the adiabatic evolution of the Vlasov-Poisson system, as was done in the classical theory [20]. However, the existence of the conserved quantities other than energy introduces additional degrees of freedom into the adiabatic evolution that eventually leads to the instability and destruction of such holes.

As a first example, we seek a solution which depends only on $z, v_{\|}$, and $R(x, y)$, where $R$ is an arbitrary function (to be defined later). Then, the first term in Eq. (85) is identically equal to zero, and in the stationary case the energy $W$ is the only integral of motion. Then, the initial velocity $v_{\|}^{(0)}$ of a particle, which at the time $t$ is located at $\mathbf{r}$ and has the velocity $v_{\|}$, is calculated from the energy as

$$
v_{\|}^{(0)}=u_{z}+\operatorname{sign}\left(v_{\|}-u_{z}\right)\left[\left(v_{\|}-u_{z}\right)^{2}-\frac{2 e}{m_{\mathrm{e}}} \varphi\right]^{1 / 2} .
$$

We notice that this solution is essentially $1 \mathrm{D}$, and the initial particle position $x^{(0)}$ may be chosen as $x^{(0)}=x$.

Conversely, for a solution which is $2 \mathrm{D}$ in a reference frame that is tilted by the angle $\theta$ relative to the $z$ axis, the time-stationary version of Eq. (85) possesses one more conserved quantity, which may be identified as the parallel electron momentum

$$
P=m_{\mathrm{e}} v_{\|}+e B_{0} x \tan \theta .
$$

Using Eqs. (87) and (91), the initial position $x^{(0)}$ now may be expressed as

$$
x^{(0)}=x-\frac{v_{\|}^{(0)}-v_{\|}}{\omega_{\text {ce }} \tan \theta},
$$

while the initial velocity is the same as for the parallel solution, Eq. (90).

We assume that the potential was adiabatically switched on at the time $t \rightarrow \infty$, and that the distribution function is determined by the propagation of particles along their perturbed trajectories. For the perturbed distribution function we use Schamel's model [20], generalized for the magnetized plasma, viz.

$$
f\left(x, v_{\|}\right)= \begin{cases}f_{\text {free }}\left(x^{(0)}, v_{\|}^{(0)}\right), & W \geqslant 0, \\ f_{\text {trap }}\left(x^{(0)}, v_{\|}^{(0)}\right), & W<0,\end{cases}
$$


The distribution function of the free particles, $f_{\text {free }}$, corresponds to a Maxwellian electron beam, which is propagating along the magnetic field with the velocity $v_{\mathrm{D}}$ relative to the background ions

$$
f_{\text {free }}\left(x^{(0)}, v_{\|}^{(0)}\right)=\left(1-\frac{\nabla_{\perp}^{2} \varphi}{B_{0} \omega_{\mathrm{ce}}}\right) \frac{n^{(0)}\left(x^{(0)}\right)}{\sqrt{2 \pi} V_{T e \|}} \exp \left[-\frac{1}{2 V_{T e \|}^{2}}\left(v_{\|}^{(0)}-v_{\mathrm{D}}\right)^{2}\right] \text {, }
$$

while that of the trapped electrons is

$$
f_{\text {trap }}\left(x^{(0)}, v_{\|}^{(0)}\right)=\left(1-\frac{\nabla_{\perp}^{2} \varphi}{B_{0} \omega_{\mathrm{ce}}}\right) \frac{n^{(0)}\left(x^{(0)}\right)}{\sqrt{2 \pi} V_{T e \|}} \exp \left\{-\frac{1}{2 V_{T e \|}^{2}}\left[\beta_{\mathrm{e}}\left(v_{\|}^{(0)}-u_{z}\right)^{2}+\left(v_{\mathrm{D}}-u_{z}\right)^{2}\right]\right\} .
$$

The distribution function (93) reduces to a shifted Maxwellian in the zero-amplitude limit, $\varphi=0$. For $\beta_{\mathrm{e}}<0$, it features an excavated vortex distribution of the trapped particles.

The electron density, obtained by the integration of the distribution function (93), is similar to that obtained in the case of an unmagnetized hole [20], with an additional term coming from the last term in Eq. (83)

$$
\frac{n_{\mathrm{e}}}{n_{0}}=1-\frac{x}{L_{n}}-\rho_{\mathrm{Le} \|}^{2} \nabla_{\perp}^{2} \phi_{\mathrm{e}}+a \phi_{\mathrm{e}}-\frac{4}{3} b \phi_{\mathrm{e}}^{3 / 2},
$$

where $\phi_{\mathrm{e}}=e \varphi / k_{\mathrm{B}} T_{\mathrm{e} \|}$ and $\rho_{\mathrm{Le} \|}=V_{T e \|} / \omega_{\mathrm{pe}}$,

$$
a=-\frac{1}{2} Z_{r}^{\prime}\left(\frac{u_{z}-v_{\mathrm{D}}}{\sqrt{2} V_{T e}}\right)
$$

where the function $Z_{r}^{\prime}$ is given in Eq. (17), and

$$
b=\frac{1}{\sqrt{\pi}}\left[1-\beta_{\mathrm{e}}-\frac{\left(v_{\mathrm{D}}-u_{z}\right)^{2}}{V_{T e \|}^{2}}\right] \exp \left[-\frac{\left(v_{\mathrm{D}}-u_{z}\right)^{2}}{2 V_{T e \|}^{2}}\right] .
$$

Likewise, by taking the first velocity moment of the distribution function (93), we may also calculate the parallel electron current. Using Ampere's law, the $z$-component of the vector potential is estimated as $A_{z} \sim\left(u_{z} / c^{2}\right) \phi_{\mathrm{e}}$, which permits us to neglect the effects of the magnetic field perturbation associated with the electron hole, since $u_{z} \sim V_{T e \|} \ll c$.

Finally, substituting the electron density, Eq. (96) into the Poisson's equation, we obtain the following equation for the electrostatic potential (where the $x / L_{n}$ term vanishes)

$$
\left[\left(r_{\mathrm{De} \|}^{2}+\rho_{\mathrm{Le} \|}^{2}\right) \nabla_{\perp}^{2}+r_{\mathrm{De} \|}^{2} \frac{\partial^{2}}{\partial z^{2}}\right] \phi_{\mathrm{e}}-a \phi_{\mathrm{e}}+\frac{4}{3} b \phi_{\mathrm{e}}^{3 / 2}=0 .
$$

This is a general equation describing an electron hole in a magnetized plasma.

For the "parallel solution", we need to use $\nabla_{\perp}^{2} \phi_{\mathrm{e}}=\left(\nabla_{\perp}^{2} R\right)\left(\mathrm{d} \phi_{\mathrm{e}} / \mathrm{d} R\right)+\left(\nabla_{\perp} R\right)^{2}\left(\mathrm{~d}^{2} \phi_{\mathrm{e}} / \mathrm{d} R^{2}\right)$, which admits various solutions for different choices of the function $R(x, y)$. Intuitively, one expects that the solutions with higher degrees of symmetry are more stable, and we will study in more details the simplest topologies. In the $1 \mathrm{D}$ slab case $\nabla_{\perp}=0$, the electron motion is not affected by the magnetic field, and consequently the parallel magnetized hole is identical to its unmagnetized counterpart [20]. Conversely, the finite perpendicular extent of the hole introduces a coupling with the magnetic field. This clearly manifests in the case of an ellipsoidal hole, described by the following parameterless spherically symmetric equation

$$
\left(\frac{\mathrm{d}^{2}}{\mathrm{~d} \zeta_{1}^{2}}+\frac{2}{\zeta_{1}} \frac{\mathrm{d}}{\mathrm{d} \zeta_{1}}\right) \phi_{\mathrm{e}}^{\prime}-\phi_{\mathrm{e}}^{\prime}+\phi_{\mathrm{e}}^{\prime 3 / 2}=0
$$

where $\phi_{\mathrm{e}}^{\prime}=(4 b / 3 a)^{2} \phi_{\mathrm{e}}$ and

$$
\zeta_{1}=\frac{1}{a^{1 / 2}}\left(\frac{x^{2}+y^{2}}{r_{\mathrm{De} \|}^{2}+\rho_{\mathrm{Le} \|}^{2}}+\frac{z^{2}}{r_{\mathrm{De} \|}^{2}}\right)^{1 / 2} .
$$




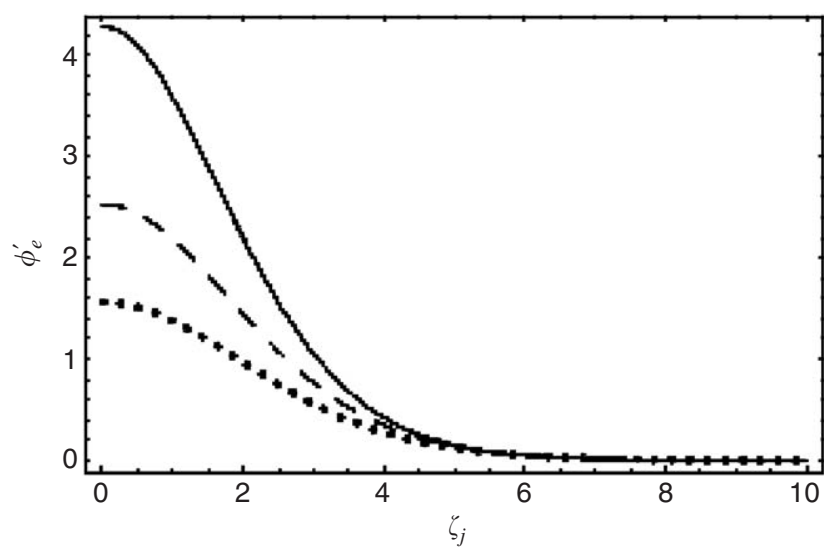

Fig. 29. The spatial profiles of the electrostatic potentials of a magnetized electron hole in an ellipsoidal (solid line; $j=1$ ), oblique cylindrical (dashed line; $j=2$ ) and oblique slab (dotted line; $j=3$ ) geometry (after Ref. [133]).

Eq. (100) is solved numerically, and the localized solution is displayed in Fig. 29. Under typical conditions in the Earth's magnetosphere, $\omega_{\mathrm{ce}}>\omega_{\mathrm{pe}}$, the perpendicular and parallel scales of such elliptical electron phase-space holes are close to each other, while in the deep magnetotail, $\omega_{\mathrm{ce}}<\omega_{\mathrm{pe}}$, the perpendicular scale is larger by the factor $\omega_{\mathrm{ce}} / \omega_{\mathrm{pe}}$. However, other parallel scales are also allowed by Eq. (99), and extensive numerical studies of the non-ellipsoidal holes would be necessary to determine the most stable structure.

The 'oblique solution' is determined by $\partial / \partial z=\tan \theta(\partial / \partial y)$. The simplest one is the oblique $1 \mathrm{D}$ slab hole, $\partial / \partial x=0$, which is identified as the nonlinear upper-hybrid mode for which Eq. (99) may be solved analytically. The solitary solution is

$$
\phi_{\mathrm{e}}^{\prime}=\frac{25}{16} \operatorname{sech}^{4}\left(\frac{\zeta_{2}}{4}\right)
$$

where

$$
\zeta_{2}=\frac{y}{a^{1 / 2}}\left[\left(1+\tan ^{2} \theta\right) r_{\mathrm{De} \|}^{2}+\rho_{\mathrm{Le} \|}^{2}\right]^{-1 / 2} .
$$

The oblique slab mode, Eq. (102) is similar to the unmagnetized 1D phase-space hole, but it is rotated by the angle $\pi / 2-\theta$, and has a somewhat larger spatial length.

Besides the slab solution, the oblique axially symmetric cylindrical hole is also possible, described by

$$
\left(\frac{\mathrm{d}^{2}}{\mathrm{~d} \zeta_{3}^{2}}+\frac{1}{\zeta_{3}} \frac{\mathrm{d}}{\mathrm{d} \zeta_{3}}\right) \phi_{\mathrm{e}}^{\prime}-\phi_{\mathrm{e}}^{\prime}+\phi_{\mathrm{e}}^{\prime 3 / 2}=0
$$

where

$$
\zeta_{3}=\frac{1}{a^{1 / 2}}\left[\frac{x^{2}}{r_{\mathrm{De} \|}^{2}+\rho_{\mathrm{Le} \|}^{2}}+\frac{y^{2}}{\left(1+\tan ^{2} \theta\right) r_{\mathrm{De} \|}^{2}+\rho_{\mathrm{Le} \|}^{2}}\right]^{1 / 2} .
$$

Such a 'cylindrical hole' has the same shape as the drift-hole, described earlier in Ref. [139], but due to its higher characteristic frequency it does not involve any ion dynamics. Its parallel spatial scale is typically $1 / \tan \theta$ times larger than the perpendicular scale. The numerical solution of Eq. (104) is shown in Fig. 29.

The characteristic electric field signal that the electron holes, Eqs. (100), (102) and (104), leave on a spacecraft is calculated as

$$
\mathbf{E} \equiv-\nabla \varphi=-\frac{k_{\mathrm{B}} T_{\mathrm{e} \|}}{e}\left(\frac{3 a}{4 b}\right)^{2} \frac{\mathrm{d} \phi_{\mathrm{e}}^{\prime}\left(\zeta_{j}\right)}{\mathrm{d} \zeta_{j}} \nabla \zeta_{j},
$$


where the subscripts $j=1,2,3$ correspond to the ellipsoidal, oblique slab and oblique cylindrical holes, respectively. As the profiles $\phi_{\mathrm{e}}^{\prime}\left(\zeta_{j}\right)$ have very similar shapes for these three types of holes, their distinction in the observational data would come from the different shapes of $\nabla \zeta_{j}$. The satellite is assumed to travel with a constant velocity $\mathbf{v}_{\text {sat }}$ along a straight-line trajectory, and its position at the time $t$ is given by

$$
\mathbf{r}=\mathbf{r}_{\perp, 0}+t \mathbf{v}_{\mathrm{sat}},
$$

where $\mathbf{r}_{\perp, 0}$ is the radius vector of the spacecraft, relative to the center of the structure, at $t=0$. Eqs. (100)-(106) are rewritten in the laboratory frame, using definitions (88) and Eq. (107). Noting that the velocity of the satellite $\left(v_{\text {sat }} \sim 10 \mathrm{~km} / \mathrm{s}\right)$ is much smaller than the parallel velocity of the structure $\left(u_{z} \sim 5000 \mathrm{~km} / \mathrm{s}\right.$ in the case of FAST events and $u_{z} \sim 100 \mathrm{~km} / \mathrm{s}$ for the GEOTAIL), for the parallel as well as for the oblique holes whose tilt angle satisfies $1 \gg \tan \theta \gg v_{\text {sat }} / u_{z}$ we have

$$
\begin{aligned}
& \zeta_{1} \approx \frac{1}{a^{1 / 2}}\left(\frac{r_{\perp, 0}^{2}}{r_{\mathrm{De} \|}^{2}+\rho_{\mathrm{Le} \|}^{2}}+\frac{t^{2} u_{z}^{2}}{r_{\mathrm{De} \|}^{2}}\right)^{1 / 2}, \\
& \nabla \zeta_{1} \approx \frac{1}{a \zeta_{1}}\left(\frac{\mathbf{r}_{\perp, 0}}{r_{\mathrm{De} \|}^{2}+\rho_{\mathrm{Le} \|}^{2}}-\frac{t u_{z} \hat{\mathbf{z}}}{r_{\mathrm{De} \|}^{2}}\right), \\
& \zeta_{2} \approx \frac{1}{a^{1 / 2}} \frac{y_{0}-t \tan \theta u_{z}}{\left(r_{\mathrm{De} \|}^{2}+\rho_{\mathrm{Le} \|}^{2}\right)^{1 / 2}}, \\
& \nabla \zeta_{2} \approx \frac{1}{a \zeta_{2}} \frac{\hat{\mathbf{y}}}{r_{\mathrm{De} \|}^{2}+\rho_{\mathrm{Le} \|}^{2}}, \\
& \zeta_{3} \approx \frac{1}{a^{1 / 2}}\left[\frac{x_{0}^{2}+\left(y_{0}-t \tan \theta u_{z}\right)^{2}}{r_{\mathrm{De} \|}^{2}+\rho_{\mathrm{Le} \|}^{2}}\right]^{1 / 2},
\end{aligned}
$$

and

$$
\nabla \zeta_{3} \approx \frac{1}{a \zeta_{3}} \frac{\mathbf{r}_{\perp, 0}-t \tan \theta u_{z} \hat{\mathbf{z}}}{r_{\mathrm{De} \|}^{2}+\rho_{\mathrm{Le} \|}^{2}} .
$$

One easily verifies from Eq. (111) that the oblique slab produces only a bipolar pulse, mainly in the perpendicular direction. Conversely, ellipsoidal and cylindrical holes produce very similar electric field signals, which are bipolar along the magnetic field, and mostly unipolar in the perpendicular direction. The ratio of the parallel and perpendicular electric field magnitudes is estimated by taking that the maximum of the bipolar pulse is located at $\zeta_{1} \sim \zeta_{3} \sim 1$, which from Eqs. (109) and (113) yields the same value for both structures

$$
\left|\frac{E_{\|}}{E_{\perp}}\right| \sim a^{1 / 2} \frac{\left(r_{\mathrm{De} \|}^{2}+\rho_{\mathrm{Le} \|}^{2}\right)^{1 / 2}}{r_{\perp, 0}} .
$$

The pulse duration is estimated in the simple case when the satellite trajectory intersects with the axis of symmetry of the structure, $r_{\perp, 0}=0$, which from Eqs. (108) and (112) yields

$$
\Delta t_{1} \sim \Delta \zeta a^{1 / 2} \frac{r_{\mathrm{De} \|}}{u_{z}}
$$

and

$$
\Delta t_{3} \sim \Delta \zeta a^{1 / 2} \frac{\left(r_{\mathrm{De} \|}^{2}+\rho_{\mathrm{Le} \|}^{2}\right)^{1 / 2}}{\tan \theta u_{z}}
$$

for the ellipsoids and cylinders, respectively, while from Fig. 29 we may use $\Delta \zeta \sim 10$. Thus, the pulse length is roughly of the order of the plasma period in the former case and $1 / \tan \theta \operatorname{times}$ larger in the latter case. These values 
are consistent with the observational data. The difference of the pulse durations for the two types of holes is relatively small, within one order of magnitude, and it is further blurred by the effects of the finite satellite velocity, and by the finite $r_{\perp, 0}$.

\subsection{Electron holes in a non-uniform magnetized plasma involving ions}

A new pseudo-3D electron hole in a non-uniform magnetized plasma is possible when the low-frequency ion dynamics is taken into account [139]. This structure is characterized as 3D electron holes coupled with hydrodynamic vortices in a quasi-neutral non-uniform magnetoplasma, and can provide a possible theoretical explanation for the POLAR and FAST satellite observations of coherent structures characterized by bipolar spikes of the parallel electric field and large perpendicular ion kinetic energies.

The high-resolution FAST diagnostics of the plasma and electromagnetic field revealed the existence of fast-moving coherent electrostatic pulses [47,48]. Their duration was typically 50-200 $\mu \mathrm{s}$, the magnitude of the wave potential energy compared with the electron kinetic energy (viz. $e \varphi \geqslant k_{\mathrm{B}} T_{\mathrm{e}} \sim 100 \mathrm{eV}$ ), and the pulses moved with the velocity close to the thermal speed of the energetic electrons $(500-5000 \mathrm{~km} / \mathrm{s})$. The electrons, originating from the ionosphere, have been accelerated by electric fields up to $10^{4}$ times their original thermal energy. The electron velocity distribution is anisotropic [47], with the parallel (to the magnetic field) temperature greatly exceeding the perpendicular, i.e. $T_{\mathrm{e}, \perp} \ll T_{\mathrm{e}, \|} \sim 30-50 \mathrm{eV}$, while the corresponding parallel electron thermal velocity, $V_{T e, \|} \sim 3000-4000 \mathrm{~km} / \mathrm{s}$, is of the same order as their streaming velocity.

Similar electrostatic pulses have also been observed in the space plasma outside the auroral acceleration region, e.g. by the GEOTAIL spacecraft measurements in the plasma-sheet boundary layer [46]. The POLAR satellite measurements in the Earth's high altitude polar magnetosphere revealed the same structures also in a higher $(5000 \mathrm{~km})$ auroral region with an upward current, with both an up-going ion stream $(\sim 1000 \mathrm{eV})$ and down-going electron stream $(\sim 800 \mathrm{eV})$. The structure was moving past the spacecraft with the velocity exceeding $100 \mathrm{~km} / \mathrm{s}$. The typical pulse duration was $0.1-2 \mathrm{~ms}$, which approaches the local $H^{+}$gyroperiod $(<10 \mathrm{~ms})$.

In the following, we discuss the properties of a new type of electron holes in a non-uniform quasineutral magnetoplasma, taking into account the ion dynamics in the low-frequency limit (i.e. when the characteristic frequency is below the ion gyrofrequency and the characteristic perpendicular scale size is larger than the ion gyroradius). In contrast to the results of Jovanović, and Pegoraro [138], where the localization resulted from the $\mathbf{E} \times \mathbf{B}$ ion fluid nonlinearity determined by the shear of the background magnetic field, Ref. [139] reported a fully nonlinear coherent solution that can be interpreted as a nonlinear drift wave coupled with an electron hole, since its localization results from the effects of the electron trapping.

In the study of nonlinear modes with the characteristic frequency below the ion gyrofrequency, one uses the fluid equations in the Padé approximation for the ions [139], and fully accounts for the electron kinetic effects associated with their motion along perturbed magnetic field lines making use of the drift-kinetic equation. One assumes a homogeneous magnetic field $\mathbf{B}$ parallel to the $z$ axis, and seeks an electrostatic solution. On the temporal and spatial scales which are much longer than the electron gyroperiod and the electron Larmor radius, one can neglect the polarization and finite Larmor radius effects, and obtain the electron density, which in the small amplitude limit $\left(e \varphi \ll k_{\mathrm{B}} T_{\mathrm{e}}\right)$ to the leading order is $[143,144]$

$$
\frac{n_{\mathrm{e}}}{n_{0}} \approx 1-\frac{x}{L_{n}}+a \phi_{\mathrm{e}}-b \frac{4}{3} \phi_{\mathrm{e}}^{3 / 2} .
$$

We assume the parallel phase velocity to be much larger than the ion-acoustic speed $c_{\mathrm{S}}=\left(k_{\mathrm{B}} T_{\mathrm{e}} / m_{\mathrm{i}}\right)^{1 / 2}\left(1+T_{\mathrm{i}} / T_{\mathrm{e}}\right)^{1 / 2}$, and regard the ions as strictly $2 \mathrm{D}$. In the frequency regime below the ion gyrofrequency $\omega_{\mathrm{ci}},|\mathrm{d} / \mathrm{d} t| \ll \omega_{\mathrm{ci}}$ and with an arbitrary ion Larmor radius, the ion response is described with the Padé approximation, see e.g. Ref. [145] and many references therein, we have

$$
\left[\frac{\partial}{\partial t}+\frac{k_{\mathrm{B}} T_{\mathrm{e}}}{e B_{0}}\left(\hat{\mathbf{z}} \times \nabla \phi_{\mathrm{e} 0}\right) \cdot \nabla\right]\left(\ln \frac{n_{\mathrm{i}}}{n_{0}}-\rho_{\mathrm{s}}^{2} \nabla_{\perp}^{2} \phi_{\mathrm{e} 0}\right)=0
$$

where $\rho_{\mathrm{s}}=V_{T e} /\left(\omega_{\mathrm{ce}} \omega_{\mathrm{ci}}\right)^{1 / 2}$ is the ion Larmor radius at the electron temperature and $\phi_{\mathrm{e} 0}=\Gamma_{0} \phi_{\mathrm{e}}$. The operator $\Gamma_{0}=\left[1-\rho_{\mathrm{s}}^{2}\left(T_{\mathrm{i}} / T_{\mathrm{e}}\right) \nabla_{\perp}^{2}\right]^{-1}$ represents the gyroradius effects. For a traveling solution, Eq. (118) is integrated once, 


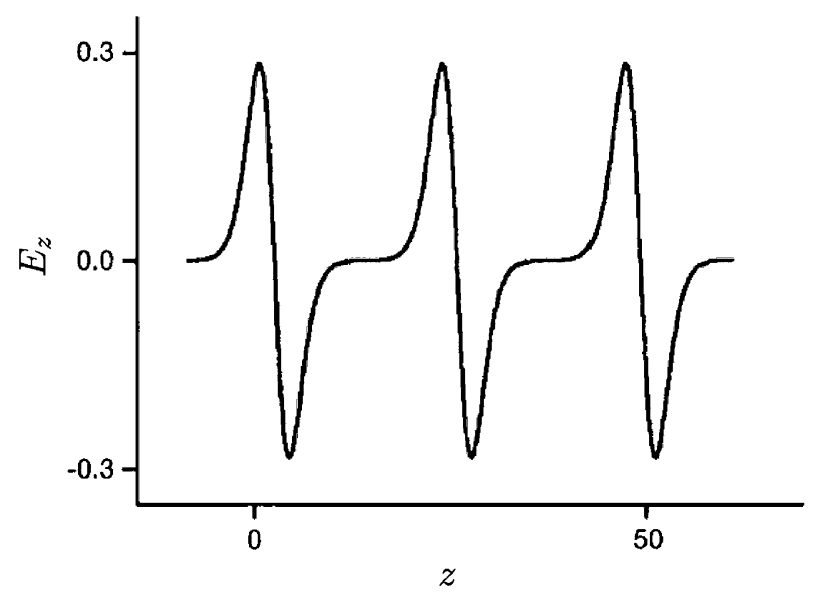

Fig. 30. The electric field of the $1 \mathrm{D}$ cnoidal wave with $\varphi_{\mathrm{e}, \min } / \psi_{\mathrm{e}}=5 \times 10^{-4}$. The electric field is normalized by $\psi_{\mathrm{e}} / l_{z}$ and space $z$ by $l_{z}=\rho_{\mathrm{s}}\left(u_{z} / u_{y}\right) /\left(a-v_{*} / u_{y}\right)^{1 / 2}$ (after Ref. [139]).

yielding

$$
\frac{n_{\mathrm{i}}}{n_{0}}-1-\rho_{\mathrm{s}}^{2} \nabla_{\perp}^{2} \phi_{\mathrm{e}}=\mathscr{G}\left(\phi_{\mathrm{e} 0}-\frac{e B_{0} u_{y}}{k_{\mathrm{B}} T_{\mathrm{e}}} x\right),
$$

where $\mathscr{G}$ is an arbitrary function of its argument. Asymptotically, for $\varphi \ll B_{0} u_{y} x, \mathscr{G}$ is a linear function, $\mathscr{G}(\xi)=\left(v_{*} / u_{y}\right) \xi$, where $v_{*}=k_{\mathrm{B}} T_{\mathrm{e}} / e B_{0} L_{n}$ is the electron diamagnetic velocity associated with the plasma inhomogeneity. For a dense plasma, $\omega_{\mathrm{pi}}^{2} / \omega_{\mathrm{ci}}^{2} \gg 1$, using the quasi-neutrality, $n_{\mathrm{e}}=n_{\mathrm{i}}$, and the asymptotic form of the function $\mathscr{G}$ on the entire $x y$-plane, we obtain from Eqs. (117) and (119)

$$
\left(1+a \frac{T_{\mathrm{i}}}{T_{\mathrm{e}}}\right) \rho_{\mathrm{s}}^{2} \nabla_{\perp}^{2} \phi_{\mathrm{e}}-\left(a-\frac{v_{*}}{u_{y}}\right) \phi_{\mathrm{e}}+b \frac{4}{3}\left(1-\frac{T_{\mathrm{i}}}{T_{\mathrm{e}}} \rho_{\mathrm{s}}^{2} \nabla_{\perp}^{2}\right) \phi_{\mathrm{e}}^{3 / 2}=0 .
$$

Eq. (120) has a similar form as Eq. (120) of Ref. [143], which describes a small amplitude 1D electron hole. However, there are significant physical differences. In our case, the dispersive effect [the first term in Eq. (120)] comes from the ion polarization and finite ion Larmor radius effects, rather than the charge separation. Subsequently, the characteristic length scale is $\rho_{\mathrm{s}}$ instead of the electron Debye radius $r_{\mathrm{De}}$. Furthermore, in the second term the coefficient $a$ is shifted by $v_{*} / u_{y}$, which introduces a new degree of freedom in the system and broadens the range of the Mach numbers $M \equiv v_{\mathrm{D}} / V_{T e}$, which admit the electron hole solution. Finally, there is a new nonlinear term coming from finite ion Larmor radius effects.

In the $1 \mathrm{D}$ case with zero ion Larmor radius, viz. $\partial / \partial x=0, T_{\mathrm{i}}=0$, Eq. (120) has the well known solitary solution

$$
\phi_{\mathrm{e}}=\psi_{\mathrm{e}} \operatorname{sech}^{4}\left[\frac{y}{\rho_{\mathrm{s}}}\left(\frac{b \psi_{\mathrm{e}}^{1 / 2}}{15}\right)^{1 / 2}\right],
$$

which is obtained if the minimum value of the potential is equal to zero, $\phi_{\mathrm{e}, \min }=0$, and the maximum value $\psi_{\mathrm{e}} \equiv \phi_{\mathrm{e}, \max }$ is related to the parameters $a$ and $b$ through $\psi_{\mathrm{e}}=\left[(15 / 16 b)\left(a-v_{*} / u_{y}\right)\right]^{2}$. If the minimum value is finite, the potential is a cnoidal wave. For $\phi_{\mathrm{e}, \min } \leqslant 10^{-2}$, the potential is in the form of a sequence of structures similar to (121) whose separation scales inversely with $\phi_{\mathrm{e}, \mathrm{min}}$. A typical periodic solution in the finite ion Larmor radius regime, a cnoidal train of 1D electron holes, is exhibited in Fig. 30. A cylindrically symmetric electron hole is shown in Fig. 31. It has the form of a long cylinder whose radius is of the order $\rho_{\mathrm{s}}$, with the pitch angle $\arctan \left(u_{y} / u_{z}\right) \ll 1$. Fig. 32 depicts a typical signal (time-dependent electric field) that is seen by an observer moving with an arbitrary velocity $\mathbf{v}_{\text {obs }}$, satisfying $\left|\mathbf{v}_{\text {obs }}\right| \ll u_{z} \leqslant V_{T e}$. It features the characteristic anti-symmetric bipolar pulse in the parallel electric field and unipolar in the perpendicular field. Anti-symmetric bipolar pulses appear in one of the perpendicular components only in the 


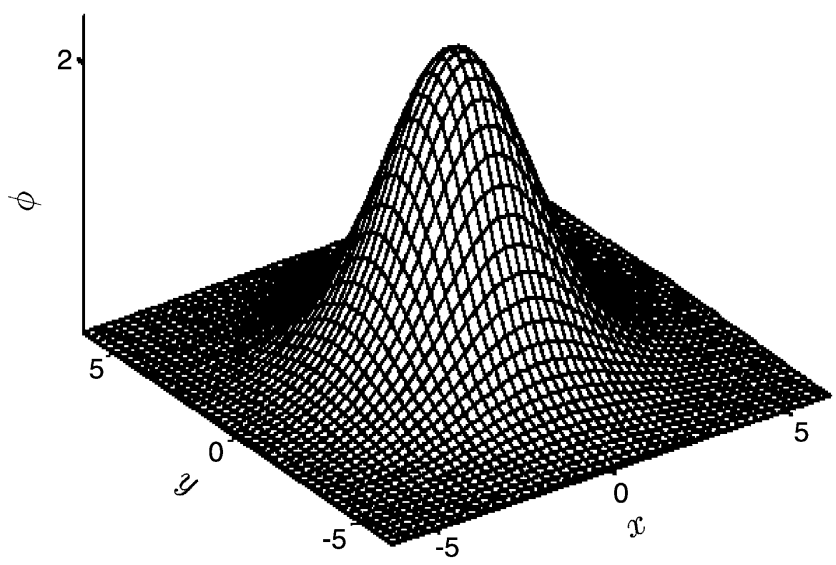

Fig. 31. The electrostatic potential of a cylindrically symmetric drift-electron-hole with $a=4 b+v_{*} / u_{y}$. The potential $\phi$ is normalized by $\left(k_{\mathrm{B}} T_{\mathrm{e}} / e\right)(3 / 4)^{2} /\left(a-v_{*} / u_{y}\right)^{2}$ and space $x$ and $x$ by $\rho_{\mathrm{S}}\left(1+a T_{\mathrm{i}} / T_{\mathrm{e}}\right)^{1 / 2} /\left(a-v_{*} / u_{y}\right)^{1 / 2}$ (after Ref. [139]).
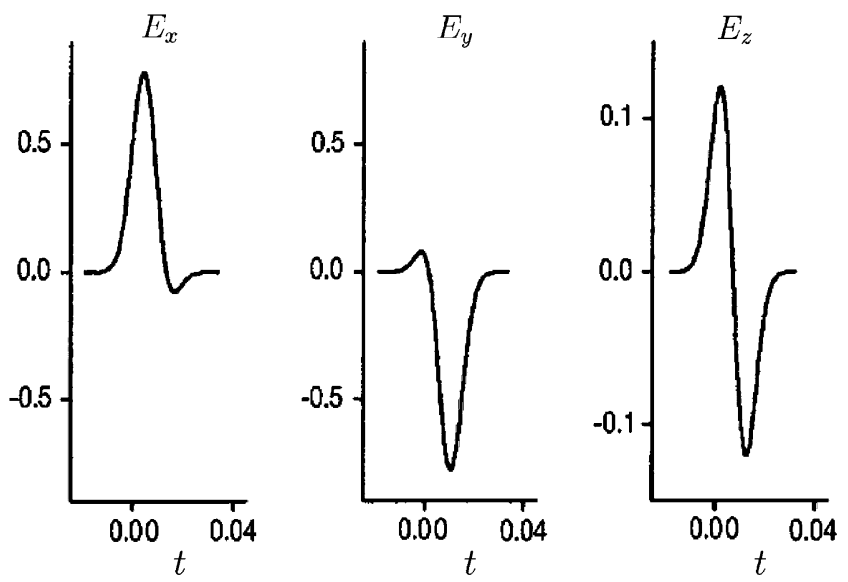

Fig. 32. Typical drift-electron-hole electric fields as observed from a spacecraft. The phase velocities of the holes are $u_{y}=0.5 c_{\mathrm{s}}$ and $u_{z}=25 c_{\mathrm{s}}$. The observer is moving with the velocity $v=0.05 c_{\mathrm{S}}$ along the trajectory $x+y=1.75, x-z=0.75$. The time $t$ is normalized by $10^{3} \rho_{\mathrm{S}} / c_{\mathrm{S}}$ and the electric field $E_{x}, E_{y}$ and $E_{z}$ by $\psi_{\mathrm{e}} / l_{z}$ where $l_{z}=\rho_{\mathrm{s}}\left(u_{z} / u_{y}\right) /\left(a-v_{*} / u_{y}\right)^{1 / 2}$ (after Ref. [139]).

statistically insignificant events in which the trajectory of the observer intersects the hole's axis of symmetry. The ratio of the parallel to the perpendicular electric field scales as $u_{y} / u_{z} \ll 1$.

In order to interpret also the POLAR and FAST events as coherent drift-hole structures, it is necessary to extend the above analysis to the frequencies comparable to the ion gyrofrequency, $|\partial / \partial t| \sim u_{y}|\partial / \partial y| \sim \omega_{\mathrm{ci}}$. A simple estimate can be obtained from the linearized ion response, including also small but finite ion Larmor radius corrections from Eq. (118). Then, one obtains

$$
\left(1+\frac{u_{y}^{2}}{\omega_{\mathrm{ci}}^{2}} \frac{\partial^{2}}{\partial y^{2}}-\frac{T_{\mathrm{i}}}{T_{\mathrm{e}}} \rho_{\mathrm{s}}^{2} \nabla_{\perp}^{2}\right) \frac{\delta n_{\mathrm{i}}}{n_{0}}=\left(\frac{v_{*}}{u_{y}}-\frac{u_{*}}{\omega_{\mathrm{ci}}} \frac{\partial}{\partial x}+\rho_{\mathrm{s}}^{2} \nabla_{\perp}^{2}\right) \phi_{\mathrm{e}} .
$$

Obviously, in the small amplitude limit, the basic (120) is not qualitatively altered if $u_{y}|\partial / \partial y|<\omega_{\text {ci. }}$. Only a spatial rescaling of the electron hole and deviation from the cylindrical symmetry [caused by the terms $\left(u_{y}^{2} / \omega_{\text {ci }}^{2}\right)\left(\partial^{2} / \partial y^{2}\right)$ and $\left.\left(u_{y} / \omega_{\mathrm{ci}}\right)(\partial / \partial x)\right]$ take place. 


\subsection{The role of phase-space holes on fast magnetic reconnection}

Computer simulations and recent observations from space plasmas reveal that phase-space vortices are common in regions where magnetic reconnection is taking place. Accordingly, it is of interest to discuss the role of trapped electrons on fast magnetic reconnection [146-148] in plasmas. The rapid reconnection of the magnetic field in the Earth's magnetosphere ("magnetic explosion") occurs on a timescale several orders of magnitude faster than the collisional and ion timescales. The signature of collisionless reconnection has been recorded by several satellite missions in different magnetospheric regions, such as the quadrupolar Hall magnetic field and the magnetic X-line at the dayside magnetopause by GEOTAIL [149] and POLAR [150], and in the magnetotail by Cluster [151]. Three-dimensional magnetic field topology in a region of solar coronal heating has also been observed [152]. Satellite observations revealed that the electron holes are commonly present in the magnetospheric regions [153] that are known to be subject to magnetic reconnection. A hole is a population of electrons that are trapped and oscillate inside a moving potential well, while the corresponding current produces a magnetic field perturbation. Likewise, 3D simulations [56] showed that collisionless reconnection in beam-plasma systems is always accompanied by the generation of electron holes or phase-space vortices. The latter are elongated cigar-shaped localized electrostatic potentials moving along the magnetic field with the velocity in the electron thermal range, making a small angle to the geomagnetic field direction.

We present here a kinetic model for collisionless magnetic reconnection, taking into account a non-isothermal electron distribution function associated with electron holes as well as the dynamics of unmagnetized ions. Specifically, we dwell on a new aspect of nonlinear magnetic reconnection in the lower-hybrid frequency regime and show that nonlinear currents and charges associated with electron phase-space vortices can efficiently mediate the topological transformation of magnetic field lines, yielding a chain of magnetic islands coupled with a double chain of hydrodynamic vortices.

We consider electromagnetic perturbations in a beam-plasma system in a sheared magnetic field. We focus on lowamplitude phenomena, viz. $e \phi / T_{\mathrm{e}} \ll 1$, that are slowly varying compared to the electron gyrofrequency, $\left|d_{t}\right| \ll \omega_{\text {ce }}$, if the radius of curvature of magnetic field lines is larger than the electron Larmor radius, $\left|\rho_{\mathrm{Le}} \nabla \times(\mathbf{B} / B)\right| \ll 1$. We restrict our analysis to plasmas with anisotropic electron temperature, $T_{\mathrm{e} \|} \gg T_{\mathrm{e} \perp}$ (the subscripts $\|$ and $\perp$ correspond to directions parallel and perpendicular to the magnetic field, respectively), e.g. due to the turbulence created by ion flow. In a low- $\beta$ plasma, $\beta_{\perp} \ll \beta_{\|} \ll 1$, compressional and torsional magnetic field perturbations can be decoupled. We consider only the torsional component and express the electric and magnetic fields as $\mathbf{E}=-\nabla \phi-\hat{\mathbf{z}} \partial A_{z} / \partial t$ and $\mathbf{B}=B_{z 0} \hat{\mathbf{z}}-\hat{\mathbf{z}} \times \nabla A_{z}$, respectively. Furthermore, we can neglect finite Larmor radius effects associated with $V_{T e \perp}$, but we keep the effects of electron polarization. The electron drift-kinetic equation then takes the simple form

$$
\left(\frac{\partial}{\partial t}+\mathbf{V} \cdot \nabla\right) g-\frac{e}{m_{\mathrm{e}}} E_{\|} \frac{\partial g}{\partial v_{\|}}=0
$$

where $\mathbf{V}=v_{\|} \hat{\mathbf{z}}+\left(1 / B_{z 0}\right) \hat{\mathbf{z}} \times \nabla\left(\phi-v_{\|} A_{z}\right)$ is the guiding center velocity, $E_{\|}=-\left[\partial / \partial z-\left(1 / B_{z 0}\right)\left(\hat{\mathbf{z}} \times \nabla A_{z}\right) \cdot \nabla\right] \phi-\partial A_{z} / \partial t$ is the parallel (to the magnetic field) component of the electric field, and $g=\log \mathscr{F}\left(v_{\|}\right)+\rho_{\mathrm{Le} \|}^{2} \nabla_{\perp}^{2}\left(e \phi / T_{\mathrm{e} \|}\right)$ where $\mathscr{F}\left(v_{\|}\right)$is the electron distribution function integrated over the velocity components perpendicular to the magnetic field. The second term in the definition of $g$ is a correction due to the divergence of the electron polarization drift.

In the same ordering, for perturbations that are fast compared to the ion gyrofrequency $\omega_{\mathrm{ci}}=e B_{z 0} / m_{\mathrm{i}}$ and whose phase velocity is much larger than the ion thermal speed, the ion dynamics is governed by

$$
\frac{\partial^{2} n_{\mathrm{i}}}{\partial t^{2}}=\frac{e n_{0}}{m_{\mathrm{i}}} \nabla^{2} \phi
$$

In the nonlinear regime, we study the case of linear shear $\mathbf{B}_{0}(x)=B_{z 0}\left[\hat{\mathbf{z}}+\hat{\mathbf{y}}\left(x-x_{0}\right) / L_{\mathrm{s}}\right]$, where $B_{z 0}=$ constant. For convenience, we work in a Galilean frame in which electrons are at rest, while the ions are moving in the $z$ direction with the constant velocity $v_{z 0} \hat{\mathbf{z}}$, related with the shear length $L_{\mathrm{S}}$ as $v_{z 0}=\left(c^{2} \epsilon_{0} / e n_{0}\right)\left(B_{z 0} / L_{\mathrm{s}}\right)$. Thus, in Eq. (124) it is necessary to introduce a Doppler shift $\partial / \partial t \rightarrow \partial / \partial t+v_{z 0} \partial / \partial z$. The vector potential is written as the sum of its background value and a perturbation, $A_{z}=-\left(B_{z 0} / 2 L_{\mathrm{s}}\right)\left[\left(x-x_{0}\right)^{2}-x_{0}^{2}\right]+\delta A_{z}$. Eq. (123) is valid when the perpendicular component of the magnetic field is small compared to $B_{z 0}$, i.e. if $\left|\nabla_{\perp} \delta A_{z}\right| \ll B_{z 0}$ and $\left(x-x_{0}\right) / L_{\mathrm{s}} \sim\left(L_{\mathrm{s}} \nabla_{\perp}\right)^{-1} \ll 1$. Magnetized plasmas, as described by Eqs. (123) and (124), are subjected to the Buneman instability due to the relative motion between electrons and ions, see Eq. (3) in Ref. [56]. In the spatial and temporal range of linearly unstable lowerhybrid waves, we seek a stationary, 2D nonlinear solution of Eqs. (123) and (124) that is travelling with the velocity 
$u_{y} \hat{\mathbf{y}}$ and making an angle $\theta$ to the $z$-axis, where $\tan \theta=u_{y} / u_{z}$. Using $\partial / \partial z=\left(u_{y} / u_{z}\right) \partial / \partial y$ and $\partial / \partial t=-u_{y} \partial / \partial y$, the drift-kinetic (123) can be written as

$$
\left\{\hat{\mathbf{z}} \times \nabla\left[\phi-v_{\|} A_{z}-x B_{z 0} u_{y}\left(1-\frac{v_{\|}}{u_{z}}\right)\right]\right\}\left[\nabla g+\frac{e}{m_{\mathrm{e}}} \frac{\partial g}{\partial v_{\|}} \frac{\nabla\left(\phi-u_{z} A_{z}\right)}{\left(v_{\|}-u_{z}\right)}\right]=0 .
$$

Eq. (125) has two integrals of motion, the energy $W=\left(m_{\mathrm{e}} / 2\right)\left(v_{\|}-u_{z}\right)^{2}-e\left(\phi-u_{z} A_{z}\right)$ and the canonical momentum $P=m_{\mathrm{e}} v_{\|}-e\left(A_{z}-x B_{z 0} u_{y} / u_{z}\right)$. If $\phi$ and $A_{z}$ were adiabatically switched on at $t=-\infty$, the initial velocity $v_{\|}^{(0)}$ and position $x^{(0)}$ of the electrons, which at time $t$ has the velocity $v_{\|}$and position $x$, are found from conserved quantities, yielding an equation for $v_{\|}^{(0)}$

$$
\left[W+u_{z} P-\frac{m_{\mathrm{e}}}{2}\left(v_{\|}^{(0)^{2}}-u_{z}^{2}\right)\right]^{2}=2 e B_{z 0} L_{\mathrm{s}} u_{y}^{2}\left(P-m_{\mathrm{e}} v_{\|}^{(0)}\right)
$$

while the initial position is given by $x^{(0)}=\left(1 / e B_{z 0} u_{y}\right)\left[W+u_{z} P-\left(m_{\mathrm{e}} / 2\right)\left(v_{\|}^{(0)^{2}}-u_{z}^{2}\right)\right]$. The electron distribution function is now obtained by replacing in the unperturbed distribution the variables $v_{\|}$and $x$ by their initial values $v_{\|}^{(0)}$ and $x^{(0)}$, provided that the evolution was adiabatic throughout its history. We consider an initially Maxwellian electron distribution with homogeneous density and temperature, and we do not make use of the characteristic $x^{(0)}$. We also adopt $x_{0}=L_{\mathrm{s}} u_{y} / u_{z}$, corresponding to a structure that is parallel to the unperturbed sheared magnetic field at $x=0$. Using explicit expressions for $W$ and $P$, Eq. (126) is written in a dimensionless form as

$$
\left(\frac{v_{\|}^{\prime 2}-v_{\|}^{\prime(0)^{2}}}{2}-\varphi+x^{\prime}\right)^{2}=2 \epsilon\left(v_{\|}^{\prime}-v_{\|}^{\prime(0)}\right)+x^{\prime 2}-2 \psi,
$$

where $x^{\prime}=x / l, v_{\|}^{\prime}=v_{\|} /\left(\omega_{\mathrm{ce}} u_{y} l\right)^{1 / 2}, \varphi=\phi /\left(B_{z 0} u_{y} l\right), \psi=\delta A_{z} L_{\mathrm{s}} /\left(B_{z 0} l^{2}\right), l$ is the typical perpendicular scale length, $\epsilon=\left(2 / \beta_{\|}\right)\left(V_{T e \|} / v_{z 0}\right)\left(\rho_{\mathrm{Le} \|}^{2} / l^{2}\right)\left(e B_{z 0} u_{y} l / T_{\mathrm{e} \|}\right)^{1 / 2}$, and $r_{\mathrm{De} \|}=V_{T e \|} / \omega_{\mathrm{pe}}$. For typical magnetized electron holes we have $\rho_{\mathrm{Le} \|}^{2} \nabla_{\perp}^{2} \sim \rho_{\mathrm{Le} \|}^{2} / l^{2} \sim\left(e \phi / T_{\mathrm{e} \|}\right)^{1 / 2}$, while the Buneman instability exists when $v_{z 0} \sim V_{\mathrm{T}, \mathrm{e} \|}$. Thus, $\epsilon$ is a small parameter if the plasma beta satisfies $1 \gg \beta_{\|} \gg e \phi / T_{\mathrm{e} \|} \sim\left(m_{\mathrm{e}} / m_{\mathrm{i}}\right)^{1 / 2}$. For larger characteristic lengths $\rho_{\mathrm{Le} \|}^{2} / l^{2} \leqslant\left(m_{\mathrm{e}} / m_{\mathrm{i}}\right)^{1 / 2}$ and relative velocities $v_{z 0} / V_{T e \|}>1$, the lower boundary of $\beta_{\|}$is further reduced.

With the accuracy to first order in the small parameter $\epsilon \ll 1$, Eq. (127) is solved as

$$
v_{\|}^{(0)}=\delta u_{\|} \pm\left[\left(v_{\|}-\delta u_{\|}\right)^{2}-2 \Phi\right]^{1 / 2},
$$

where $\Phi=\varphi-x \pm\left(x^{2}-2 \psi\right)^{1 / 2}$ and $\delta u_{\|}= \pm \epsilon\left(x^{2}-2 \psi\right)^{-1 / 2}$. The signs in front of the square roots are adopted so that for $\left|v_{\|}\right| \gg 1$ and $|x| \gg 1$ we have $\pm\left(v_{\|}^{2}-2 \Phi\right)^{1 / 2} \approx v_{\|}$and $\pm\left(x^{2}-2 \psi\right)^{1 / 2} \approx x$, respectively, and the sign reversals take place in branch points. One pair of branch points, $v_{\Phi 1}, v_{\Phi_{2}}$ corresponds to the usual trapping by an electrostatic potential $v_{\Phi 1, \Phi 2}=\delta u_{\|} \pm(2 \Phi)^{1 / 2}$, and their effect will be referred to as "electrostatic trapping". The second pair of branch points cannot be determined from Eq. (128) since it is not valid when $x^{2}-2 \psi$ is small. A simple inspection reveals that $\delta u_{\|}$is the phase velocity along the perturbed magnetic field, and Eq. (128) is interpreted as the initial electron velocity in the stationary electrostatic potential $\Phi$ moving with the phase velocity $\delta u_{\|} \hat{\mathbf{z}}$. Such picture breaks down in the region where the structure is almost parallel to magnetic field, i.e. for $\left|x^{2}-2 \psi\right| \leqslant \epsilon^{2}$, where the expansion in $\epsilon$ does not converge.

For particles that are non-resonant with respect to the electrostatic trapping, Eq. (127) is solved using the small parameter $\left(v_{\|}-v_{\|}^{(0)}\right) / v_{\|} \sim e \phi / T_{\mathrm{e} \|} \ll 1$, yielding

$$
v_{\|}^{(0)}= \pm\left(v_{\|}^{2}-2\left\{\tilde{\phi} \pm\left[\tilde{\phi}^{2}-(\varphi-x)^{2}+x^{2}-2 \psi\right]^{1 / 2}\right\}\right)^{1 / 2},
$$

where $\tilde{\phi}=\varphi-x+\epsilon / v_{\|}$. It can be seen from Eqs. (128) and (129) that for a localized solution the total vector potential is negative on most of the $x, y$ plane, $\psi-x^{2} / 2<0$. Finite size area where the vector potential is positive corresponds 
to magnetic islands in which the lines of the magnetic field $\mathbf{B}_{\perp}$ close on themselves, and the contour $\psi-x^{2} / 2=0$ is the magnetic separatrix between open and closed magnetic field lines. Besides the electrostatic branch points, Eq. (129) has two more branch points coming from the zeros of the innermost square root, $v_{B 1}=-\epsilon / \Psi$ and $v_{B 2}=\epsilon \Psi /\left(2 \psi-x^{2}\right)$, where $\Psi=\varphi-x+\operatorname{sign}(\varphi-x)\left[(\varphi-x)^{2}+2 \psi-x^{2}\right]^{1 / 2}$. When $\left|x^{2}-2 \psi\right| \sim 1$ they are both inside the electrostatic resonant region $\left(v_{\Phi 1}, v_{\Phi 2}\right)$, very close to the origin $v_{\|}=0$, and their effects are likely to be overcome by those of electrostatic trapping. Conversely, when $\left|x^{2}-2 \psi\right| \leqslant \epsilon$ and the approximation Eq. (128) is not applicable, $v_{B 1}$ remains small but $v_{B 2}=2 \epsilon(\varphi-x) /\left(2 \psi-x^{2}\right)$ and it lies outside of the electrostatic trapping region, yielding new resonant particle effects. In particular, in the fluid picture the parallel current has a singularity at the magnetic separatrix that may be limited by these new particle resonance. The effects of branch points $v_{B 1}$ and $v_{B 2}$ will be referred to as "magnetic trapping".

The memory of the initial velocity of electrostatically trapped particles is likely to be lost due to many oscillations inside the trapping potential and their distribution is Gaussian with a different temperature (possibly negative) than that of free particles. As a working hypothesis, we assume that the same is true also for magnetic trapping, and use the standard distribution function [20], modified to account for the electron polarization. We have

$$
\mathscr{F}\left(v_{\|}\right)=\frac{n_{0}}{(2 \pi)^{1 / 2} V_{T e \|}} \exp \left\{-\frac{1}{2 V_{T e \|}^{2}}\left[\left(\operatorname{Re} v_{\|}^{(0)}\right)^{2}-\beta_{\mathrm{e}}\left(\operatorname{Im} v_{\|}^{(0)}\right)^{2}+\frac{2 e}{m_{\mathrm{e}}} \rho_{\mathrm{Le} \|}^{2} \nabla_{\perp}^{2} \phi\right]\right\}
$$

If there are two or more non-overlapping regions of trapped particles in which $\operatorname{Im} v_{\|}^{(0)} \neq 0$, we may assign a different trapping parameter $\beta_{\mathrm{e}}$ to each of them. The real and imaginary parts of the initial particle velocity $v_{\|}^{(0)}$ are calculated using the small parameters that are characteristic for the regions of magnetically and electrostatically trapped, as well as of the free particles. For the free and electrostatically trapped particles, neglecting the effects of magnetic trapping and with the accuracy to leading orders in $\epsilon$ and $\Phi$, the renormalized phase velocity reduces to $\overline{\delta u_{\|}} \rightarrow \delta u_{\Phi}$, where $\delta u_{\Phi}= \pm \epsilon\left(x^{2}-2 \psi\right)^{3 / 2} /\left[\left(x^{2}-2 \psi\right)^{2}+\left(\epsilon^{2} / 2\right) \Phi\right]$. While on open magnetic field lines the quantities $\Phi$ and $\delta u_{\Phi}$ are real, inside magnetic islands they become complex. Conversely, inside the magnetic trapping region but outside electrostatic trapping region, with the same accuracy we have $\operatorname{Re} v_{\|}^{(0)}=v_{\|}-\Phi /\left(v_{\|}-\delta u_{B}\right)$ and $\operatorname{Im} v_{\|}^{(0)}= \pm\left(1 / v_{\|}\right)\left[-\left(2 \epsilon / v_{\|}\right)(\varphi-\right.$ $\left.x)^{2}+2 \psi-x^{2}\right]^{1 / 2}$, where $\delta u_{B}=-\epsilon /(\varphi-x)$ is the effective phase velocity in this region.

The distribution function (130) is integrated over velocity space, yielding the electron density and and parallel velocity to leading order

$$
N_{\mathrm{e}}=1-\frac{\lambda_{\mathrm{Le} \|}^{2} \nabla_{\perp}^{2} \varphi}{\mathscr{T}_{\mathrm{e} \|}}+\frac{\hat{\Phi}}{\mathscr{T}_{\mathrm{e} \|}}-b_{\Phi}\left(\frac{\hat{\Phi}}{\mathscr{T}_{\mathrm{e} \|}}\right)^{3 / 2}
$$

and

$$
V_{z, e}=\operatorname{Re} \delta u_{\Phi} \frac{\hat{\Phi}}{\mathscr{T}_{\mathrm{e} \|}}-w_{\Phi}\left(\frac{\hat{\Phi}}{\mathscr{T}_{\mathrm{e} \|}}\right)^{3 / 2}+w_{B}\left|\frac{\hat{\Phi}}{T_{\mathrm{e} \|}}\right|^{1 / 2}
$$

respectively. Here, we use the notation $\hat{\Phi}=\operatorname{Re} \Phi$ and the normalization $N_{\mathrm{e}}=n_{\mathrm{e}} / n_{0}$ and $\mathscr{T}_{\mathrm{e} \|}=T_{\mathrm{e} \|} /\left(e B_{z 0} u_{y} l\right)$. The velocities $V_{z, \mathrm{e}}$ and $\delta u_{\Phi}$ are normalized by $\left(\omega_{\mathrm{ce}} u_{y} l\right)^{1 / 2}$ and the space $x, y, z$ and by $l$ and we use the normalization $\lambda_{\mathrm{Le} \|}=\rho_{\mathrm{Le} \|} / l$ and $\lambda_{\mathrm{De} \|}=r_{\mathrm{De} \|} / l$ of the Larmor and Debye radius, respectively. The other normalizations are the same as in Eq. (127). The parameters $b_{\Phi}$ and $w_{\Phi}$ arise from the contributions of particles that are in the electrostatic resonance (both trapped and marginally free), $b_{\Phi}=\pi^{-1 / 2}\left(1-\beta_{\Phi}\right)\left(2 / 3+\tau_{b}|\Phi| / \operatorname{Re} \Phi\right)$ and $w_{\Phi}=\pi^{-1 / 2}(4 / 3)(1-$ $\left.\left.\beta_{\Phi}\right)\left[\operatorname{Re} \delta u_{\Phi} \pm \tau_{w} \operatorname{Im} \delta u_{\Phi} \operatorname{Im} \Phi /|\Phi|\right)\right]$. Inside magnetic islands all these particles are regarded as deeply trapped, taking $V_{\|}^{2} \gg 2 \operatorname{Re} \Phi>0$ where $V_{\|}=v_{\|} /\left(\omega_{\mathrm{ce}} u_{y} l\right)^{1 / 2}$. The coefficients $\tau_{b}$ and $\tau_{w}$ are fitted so as to account for the contribution of marginally trapped particles taking $\tau_{b}=\tau_{w}=1$ inside magnetic islands and $\tau_{b}=2 / 3$ outside. The last term in Eq. (132), where $w_{B}=\epsilon \beta_{B}[\xi(1-\log \xi)-1] /\left(2 \pi^{1 / 2} \mathscr{V}_{T e \|}^{2}\right)$ where $\mathscr{V}_{T e \|}=V_{T e \|} /\left(\omega_{\mathrm{ce}} u_{y} l\right)^{1 / 2}$ and $\xi=\left(x^{2}-2 \psi\right) /\left[\epsilon(2 \operatorname{Re} \Phi)^{1 / 2}\right]$, exists only for $0<\xi<1$ and it represents the only contribution of the magnetic resonance. 

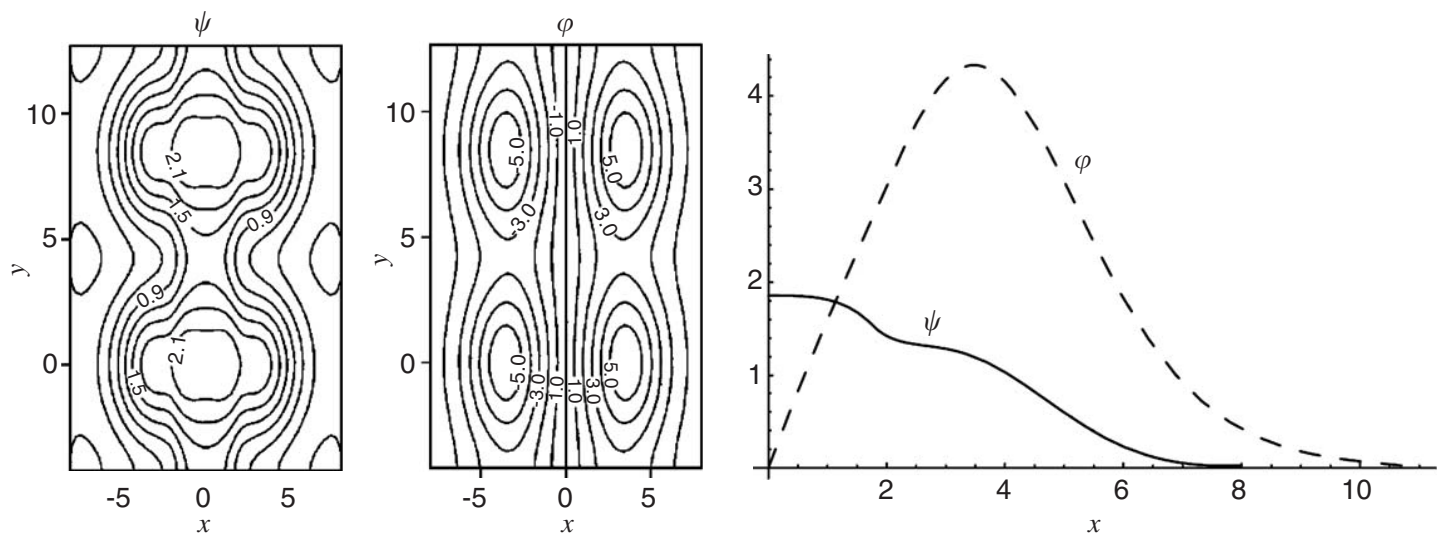

Fig. 33. Surface plots of the vector potential $\psi$ (left panel) and the scalar potential $\varphi$ (middle panel), and the transverse profiles (right panel) at $y=0$ of the vector potential $\psi$ (solid line) and electrostatic potential $\varphi$ (dashed line) of a thin magnetic island with $\partial / \partial x>\partial / \partial y$. The normalizations are explained in the text, see. Eqs. (127) and (132). We have adopted $a_{\varphi}=0.01, \epsilon=0.2, \alpha=0.4, \beta_{\Phi}=1-0.45 \pi^{1 / 2} \mathscr{T}$ e, and $\beta_{\mathrm{B}}=1+0.25 \pi^{1 / 2} \mathscr{T}$ e (after Refs. [147] and [148]).

Our system of equations is closed by the dimensionless Poisson's equation $N_{\mathrm{e}}-N_{\mathrm{i}}=\left(\lambda_{\mathrm{De} \|} / l\right)^{2}\left[\mathrm{\partial}^{2} / \partial x^{2}+(1+\right.$ $\left.\left.u_{y}^{2} / u_{z}^{2}\right) \partial^{2} / \partial y^{2}\right]\left(\varphi / \mathscr{T}_{\mathrm{e} \|}\right)$, where $N_{\mathrm{i}}=n_{\mathrm{i}} / n_{0}$, and Ampere's law $V_{z, \mathrm{e}}=\epsilon \alpha^{2} \nabla_{\perp}^{2}\left(\psi / \mathscr{T}_{\mathrm{e} \|}\right)$, where $\alpha=\left(V_{T e \|} / u_{y}\right)$ $\left(v_{z 0} / c\right)\left(\omega_{\mathrm{pe}} / \omega_{\mathrm{ce}}\right)$, yielding

$$
\begin{aligned}
& \frac{\partial^{2}}{\partial y^{2}}\left\{\left[\left(\lambda_{\mathrm{De} \|}^{2}+\lambda_{\mathrm{Le} \|}^{2}\right) \nabla_{\perp}^{2}+a_{\varphi}\right] \frac{\varphi}{\mathscr{T}_{\mathrm{e} \|}}-\frac{\hat{\Phi}}{\mathscr{T}_{\mathrm{e} \|}}+b_{\Phi}\left(\frac{\hat{\Phi}}{\mathscr{T}_{\mathrm{e} \|}}\right)^{\frac{3}{2}}\right\} \\
& \quad=-\frac{m_{\mathrm{e}}}{m_{\mathrm{i}}} \frac{\mathscr{V}_{T e \|}^{2}}{u_{y}^{2}\left(1-v_{z 0} / u_{z}\right)^{2} \mathscr{T}_{\mathrm{e} \|}} \frac{\partial^{2} \varphi}{\partial x^{2}}
\end{aligned}
$$

and

$$
\frac{\epsilon \alpha^{2}}{\mathscr{T}_{\mathrm{e} \|}} \nabla_{\perp}^{2} \psi=\operatorname{Re} \delta u_{\Phi} \frac{\hat{\Phi}}{\mathscr{T}_{\mathrm{e} \|}}-w_{\Phi}\left(\frac{\hat{\Phi}}{\mathscr{T}_{\mathrm{e} \|}}\right)^{\frac{3}{2}}+w_{B}\left(\frac{\hat{\Phi}}{\mathscr{T}_{\mathrm{e} \|}}\right)^{\frac{1}{2}}
$$

where $a_{\varphi}=\left(m_{\mathrm{e}} / m_{\mathrm{i}}\right)\left(V_{T e \|}^{2} / u_{y}^{2}\right)\left(1+u_{y}^{2} / u_{z}^{2}\right) /\left(1-v_{z 0} / u_{z}\right)^{2}$, and we assumed $u_{z} \neq v_{z 0}$.

Eqs. (133) and (134) are solved numerically for a thin magnetic island, $\partial / \partial x \gg \partial / \partial y$, and the solutions are displayed in Fig. 33. We see that the vector potential $\psi$ is almost symmetric while the potential $\varphi$ is anti-symmetric, corresponding to a single chain of magnetic islands and double chain of fluid vortices, created by the symmetric distribution of trapped particle charges and current inside the island and at the separatrix, but there may exist a small asymmetry in the outside region due to the electron trapping in the positive electrostatic potential. The magnetic separatrix is located at $x \approx 1.8$. The size of the island is much larger than the electron Larmor radius $\rho_{\text {Le }}$, and it is estimated as $l \sim 3.6 \rho_{\mathrm{Le}}\left(e \phi / T_{\mathrm{e} \|}\right)^{1 / 4} \gg \rho_{\mathrm{Le}}\left(m_{\mathrm{e}} / m_{\mathrm{i}}\right)^{1 / 4}$. The characteristic wavelength of the structure along the $y$-axis is much larger than $l$.

\section{Nonlinear interactions between high-frequency waves and holes}

In space plasmas, large amplitude waves can exist in association with electrostatic phase-space holes. Here, we consider nonlinear interactions between high-frequency electrostatic and electromagnetic waves with phase-space holes. Since both ion and electron holes are associated with a local depletion of the electron density, they can scatter waves and can also work as a resonance cavity in which waves can be trapped. We will here discuss the trapping of Langmuir waves in ion holes [111] including the influence of dust on the ion holes [154]. A similar scenario of the 
trapping of Langmuir waves in electron holes were discussed in Ref. [69]. We will conclude the section by discussing the possibility of trapping of intense electromagnetic waves in electron holes [155], where relativistic effects play an important role in the formation and dynamics of the electron holes.

\subsection{Trapping of Langmuir waves in ion holes}

Let us suppose that our plasma contains finite amplitude Langmuir waves which interact nonlinearly with the ambient plasma. The interaction is governed by

$$
\begin{aligned}
& \frac{\partial n_{\mathrm{eh}}}{\partial t}+\frac{\partial}{\partial x}\left(n_{\mathrm{es}} u_{\mathrm{h}}\right)=0, \\
& \frac{\partial u_{\mathrm{h}}}{\partial t} \approx-\frac{e}{m_{\mathrm{e}}} E_{\mathrm{h}}-\frac{3 V_{T e}^{2}}{n_{\mathrm{e} 0}} \frac{\partial n_{\mathrm{eh}}}{\partial x}
\end{aligned}
$$

and

$$
\frac{\partial E_{\mathrm{h}}}{\partial x}=-\frac{e}{\epsilon_{0}} n_{\mathrm{eh}}
$$

where $n_{\mathrm{eh}}$ and $u_{\mathrm{h}}$ are the high-frequency perturbations of the electron number density and electron velocity associated with the Langmuir waves, $n_{\mathrm{es}}$ is the electron number density of the plasma slow motion and $E_{\mathrm{h}}$ is the Langmuir wave electric field.

Eqs. (135)-(137) can be combined to obtain the Langmuir wave equation in the presence of fully nonlinear lowfrequency (in comparison with the Langmuir wave frequency) density perturbations. We have

$$
\frac{\partial^{2} E_{\mathrm{h}}}{\partial t^{2}}-3 V_{T e}^{2} \frac{\partial^{2} E_{\mathrm{h}}}{\partial x^{2}}+\omega_{\mathrm{pe}}^{2} N_{\mathrm{e}} E_{\mathrm{h}}=0
$$

where $N_{\mathrm{e}}=n_{\mathrm{e} s} / n_{\mathrm{e} 0}$. The envelope of the Langmuir wave electric field is assumed to vary on a timescale slower than the electron plasma period. Hence, introducing $E_{\mathrm{h}}=(1 / 2) E(t, x) \exp \left(-\mathrm{i} \omega_{0} t+\mathrm{i} k_{0} x\right)+$ complex conjugate, and invoking the WKB ansatz, viz. $\partial E / \partial t \ll \omega_{0} E$, we obtain from (138) the equation for the Langmuir wave envelope

$$
2 \mathrm{i} \omega_{0}\left(\frac{\partial}{\partial t}+v_{\mathrm{g}} \frac{\partial}{\partial x}\right) E+3 V_{T e}^{2} \frac{\partial^{2} E}{\partial x^{2}}+\omega_{\mathrm{pe}}^{2}\left(1-N_{\mathrm{e}}\right) E=0
$$

where $\omega_{0}=\left(\omega_{\mathrm{pe}}^{2}+3 k_{0} V_{T e}^{2}\right)^{1 / 2}$ is the Bohm-Gross frequency and $v_{\mathrm{g}}=3 k_{0} V_{T e}^{2} / \omega_{0} \approx 3 k_{0} V_{T e}^{2} / \omega_{\mathrm{pe}}$ is the group velocity of the Langmuir wave.

We note that Eq. (139) has been derived by combining the electron continuity and momentum equations as well as by using Poisson's equation with fixed ions, and by retaining the arbitrary large electron number density variation $n_{\mathrm{e}}$ associated with the ion holes in the presence of the Langmuir wave ponderomotive force. Assuming that the phase speeds of the ion holes are much smaller than the electron thermal speed, we obtain the electron number density in the presence of the ponderomotive force of the Langmuir waves from the inertialess electron equation of motion. The result is

$$
N_{\mathrm{e}}=\exp \left[\tau\left(\phi_{\mathrm{i}}-W^{2}\right)\right],
$$

where $\tau=T_{\mathrm{i}} / T_{\mathrm{e}}, W=\left(\epsilon_{0}|E|^{2} / 4 n_{0} k_{\mathrm{B}} T_{\mathrm{i}}\right)^{1 / 2}$ is the normalized Langmuir wave envelope, and $\phi_{\mathrm{i}}=e \varphi / k_{\mathrm{B}} T_{\mathrm{i}}$ is the normalized electrostatic potential of the ion hole. We note that the $W^{2}$-term in Eq. (140) comes from the averaging of the nonlinear term $m_{\mathrm{e}} v_{\text {he }} \partial v_{\text {he }} / \partial x$ over the Langmuir wave period $2 \pi / \omega_{\text {pe }}$, where $v_{\text {he }} \approx-e E_{\mathrm{h}} / m_{\mathrm{e}} \omega_{\text {pe }}$ is the highfrequency electron quiver velocity in the Langmuir wave electric field $E_{l}$.

If the potential has a maximum $\phi_{i, \max }>0$, then there exist, in general, trapped ions where $\phi_{\mathrm{i}}<\phi_{\mathrm{i}, \max }$, while at the point where $\phi_{\mathrm{i}}=\phi_{\mathrm{i}, \max }$ there are no trapped ions. Similar to Schamel [22], we chose at this point a Maxwellian distribution for the free ions. The ion distribution function associated with the ion holes can then be obtained by solving the ion Vlasov equation for free and trapped ions, which have speeds larger and smaller than $\left[2\left(\phi_{\mathrm{i}, \max }-\phi_{\mathrm{i}}\right)\right]^{1 / 2}$, respectively. The electric potential will turn out to be essentially negative, with only a small-amplitude positive maximum 
$\phi_{\mathrm{i}, \max }$ compared to the large-amplitude negative potential well with a minimum at $\phi_{\mathrm{i}, \min } \equiv-\psi$. Thus, the potential is restricted by $-\psi \leqslant \phi_{\mathrm{i}} \leqslant \phi_{\mathrm{i} \text {,max }}$, where $\psi$ plays the role of the amplitude. Integrating the sum of the free and trapped ion distribution functions over velocity space, we obtain the ion number density [22]

$$
N_{\mathrm{i}}=b \exp \left(-\frac{M_{\mathrm{i}}^{2}}{2}\right)\left[I\left(-\Phi_{\mathrm{i}}\right)+\kappa\left(\frac{M_{\mathrm{i}}^{2}}{2},-\Phi_{\mathrm{i}}\right)+\frac{2}{\sqrt{\pi\left|\beta_{\mathrm{i}}\right|}} W_{\mathrm{D}}\left(\sqrt{\beta_{\mathrm{i}} \Phi_{\mathrm{i}}}\right)\right],
$$

where $N_{\mathrm{i}}=n_{\mathrm{i}} / n_{0}, M_{\mathrm{i}}=u_{\mathrm{i} 0} / V_{T \mathrm{i}}$ is the Mach number, $u_{\mathrm{i} 0}$ is the ion hole speed, $\Phi_{\mathrm{i}}=\phi_{\mathrm{i}}-\phi_{\mathrm{i}, \max }$, and where the special functions $I, \kappa$ and $W_{\mathrm{D}}$ are given by Eqs. (11)-(13). The normalization constant $b$ is chosen so that the total density of the ions is $n_{0}$ where $\phi_{\mathrm{i}}=0$, i.e.

$$
b=\exp \left(\frac{M_{\mathrm{i}}^{2}}{2}\right)\left\{I\left(\phi_{\mathrm{i}, \text { max }}\right)+\kappa\left(\frac{M_{\mathrm{i}}^{2}}{2}, \phi_{\mathrm{i}, \max }\right)+\frac{2}{\sqrt{\pi\left|\beta_{\mathrm{i}}\right|}} W_{\mathrm{D}}\left(\sqrt{-\beta_{\mathrm{i}} \phi_{\mathrm{i}, \max }}\right)\right\}^{-1} .
$$

We are here mostly interested in negative values of $\beta_{\mathrm{i}}$; however, for positive $\beta_{\mathrm{i}}$, we use [156] $W_{\mathrm{D}}(\mathrm{i} Z)=\mathrm{i}(\sqrt{\pi} / 2)$ $\times \exp \left(Z^{2}\right) \operatorname{erf}(Z)($ where $\mathrm{i}=\sqrt{-1})$ and replace the term $\left(2 / \sqrt{\pi\left|\beta_{\mathrm{i}}\right|}\right) W_{\mathrm{D}}\left(\sqrt{\beta_{\mathrm{i}} \Phi_{\mathrm{i}}}\right)$ in Eq. (141) by $\left(1 / \sqrt{\beta_{\mathrm{i}}}\right) \exp \left(-\beta_{\mathrm{i}} \Phi_{\mathrm{i}}\right)$ $\times \operatorname{erf}\left[\sqrt{-\beta_{\mathrm{i}} \Phi_{\mathrm{i}}}\right]$; we note especially that $M_{\mathrm{i}}=0, \beta_{\mathrm{i}}=1$ leads to a Boltzmann distribution $n_{\mathrm{i}}=n_{0} \exp \left(-\phi_{\mathrm{i}}\right)$ for the ion number density. The Langmuir wave ponderomotive force acting on the ions is weaker by the electron to ion mass ratio in comparison with the one acting on the electrons, and therefore it is ignored in Eq. (141). The electron ponderomotive force is transmitted to ions via the ambipolar potential $\phi_{i}$, which is determined from Poisson's equation

$$
\tau r_{\mathrm{De}}^{2} \frac{\partial^{2} \phi_{\mathrm{i}}}{\partial x^{2}}=N_{\mathrm{e}}-N_{\mathrm{i}}
$$

We are interested in quasi-steady state solutions of Eqs. (139)-(143), which are fully nonlinear. We insert $E(x, t)=$ $W(\xi) \exp [\mathrm{i}(K x-\Theta t)]$ and $\phi_{\mathrm{i}}=\phi_{\mathrm{i}}(\xi)$, where $\xi=\left(x-u_{0} t\right) / r_{\text {De }}$, and where $W(\xi), K$ and $\Theta$ are assumed to be real, into Eqs. (139)-(143) and obtain a coupled set of nonlinear equations

$$
3 \frac{\partial^{2} 2 W}{\partial \xi^{2}}-(\lambda-1) W-W \exp \left[\tau\left(\phi_{\mathrm{i}}-W^{2}\right)\right]=0,
$$

where $\lambda=-2 \omega_{\mathrm{pe}}^{-1} \Theta-3 k^{2} r_{\mathrm{De}}^{2}\left(1-u_{0}^{2} / v_{\mathrm{g}}^{2}\right)=-2 \omega_{\mathrm{pe}}^{-1} \Theta-3 k^{2} r_{\mathrm{De}}^{2}+u_{0}^{2} / 3 V_{T e}^{2}$ represents a nonlinear frequency shift, and

$$
\begin{aligned}
& \tau \frac{\partial^{2} \phi_{\mathrm{i}}}{\partial \xi^{2}}-\exp \left[\tau\left(\phi_{\mathrm{i}}-W^{2}\right)\right]+b \exp \left(-\frac{M_{\mathrm{i}}^{2}}{2}\right) \\
& \quad \times\left\{I\left(-\Phi_{\mathrm{i}}\right)+\kappa\left(\frac{M_{\mathrm{i}}^{2}}{2},-\Phi_{\mathrm{i}}\right)+\frac{2}{\sqrt{\pi\left|\beta_{\mathrm{i}}\right|}} W_{\mathrm{D}}\left(\sqrt{\beta_{\mathrm{i}} \Phi_{\mathrm{i}}}\right)\right\}=0 .
\end{aligned}
$$

The system of Eqs. (144) and (145) admits the first integral in the form of a Hamiltonian

$$
H\left(W, \phi_{\mathrm{i}} ; M_{\mathrm{i}}, \tau, \beta_{\mathrm{i}}, \lambda\right)=3\left(\frac{\partial W}{\partial \xi}\right)^{2}-\frac{\tau}{2}\left(\frac{\partial \phi_{\mathrm{i}}}{\partial \xi}\right)^{2}+V\left(\phi_{\mathrm{i}}, W, M_{\mathrm{i}}, \beta_{\mathrm{i}}, \tau\right)=0,
$$

where the 'potential energy' is

$$
\begin{aligned}
V\left(\phi_{\mathrm{i}}, W, M_{\mathrm{i}}, \beta_{\mathrm{i}}, \tau\right)= & (1-\lambda) W^{2}+\frac{1}{\tau}\left\{\exp \left[\tau\left(\phi_{\mathrm{i}}-W^{2}\right)\right]-1\right\} \\
& +b \exp \left(-\frac{M_{\mathrm{i}}^{2}}{2}\right)\left[P\left(-\Phi_{\mathrm{i}}, \beta_{\mathrm{i}}\right)-P\left(\phi_{\mathrm{i}, \max }, \beta_{\mathrm{i}}\right)\right. \\
& \left.+h\left(\frac{M_{\mathrm{i}}^{2}}{2}, 0,-\Phi_{\mathrm{i}}\right)-h\left(\frac{M_{\mathrm{i}}^{2}}{2}, 0, \phi_{\mathrm{i}, \max }\right)\right]=0,
\end{aligned}
$$


where in the unperturbed state $(|\xi|=\infty)$ we have used the boundary conditions $W=0, \phi_{\mathrm{i}}=0, \partial W / \partial \xi=0, \partial \phi_{\mathrm{i}} / \partial \xi=0$. The auxiliary functions are defined as [26]

$$
P\left(\Phi, \beta_{\mathrm{i}}\right)=I(\Phi)+2 \sqrt{\frac{\Phi}{\pi}}\left(1-\beta_{\mathrm{i}}^{-1}\right)+\frac{2}{\beta_{\mathrm{i}} \sqrt{\pi\left|\beta_{\mathrm{i}}\right|}} W_{\mathrm{D}}\left(\sqrt{-\Phi \beta_{\mathrm{i}}}\right)
$$

and

$$
h(X, a, b)=\int_{a}^{b} \kappa(X, \Phi) \mathrm{d} \Phi .
$$

For symmetric solutions defined by $W(\xi)=W(-\xi)$ and $\phi_{\mathrm{i}}(\xi)=\phi_{\mathrm{i}}(-\xi)$, the appropriate boundary conditions at $\xi=0$ are $W=W_{0}, \phi_{\mathrm{i}}=-\psi_{\mathrm{i}}, \partial W / \partial \xi=0$, and $\partial \phi_{\mathrm{i}} / \partial \xi=0$. Hence, from Eq. (147) we have

$$
\begin{aligned}
& (\lambda-1) W_{0}^{2}-\frac{1}{\tau}\left\{\exp \left[\tau\left(-\psi_{\mathrm{i}}-W_{0}^{2}\right)\right]-1\right\}-b \exp \left(-\frac{M_{\mathrm{i}}^{2}}{2}\right)\left[P\left(\phi_{\mathrm{i}, \max }+\psi_{\mathrm{i}}, \beta_{\mathrm{i}}\right)-P\left(\phi_{\mathrm{i}, \max }, \beta_{\mathrm{i}}\right)\right. \\
& \left.+h\left(\frac{M_{\mathrm{i}}^{2}}{2}, 0, \phi_{\mathrm{i}, \max }+\psi_{\mathrm{i}}\right)-h\left(\frac{M_{\mathrm{i}}^{2}}{2}, 0, \phi_{\mathrm{i}, \max }\right)\right]=0,
\end{aligned}
$$

which shows how the maximum values of $W_{0}$ and $\psi_{\mathrm{i}}+\phi_{\mathrm{i}, \max }$ are related to $M_{\mathrm{i}}$ for given values of $\lambda, \tau$ and $\beta_{\mathrm{i}}$. Eq. (150) may therefore be regarded as a nonlinear dispersion relation for the coupled ion hole-Langmuir envelope system. A practical application of Hamiltonian (147) is to check the correctness of any numerical scheme used to solve Eqs. (144) and (145).

Ion holes without large-amplitude Langmuir waves have only negative potentials (i.e. $\phi_{i, \max }=0$,) and are governed by the energy integral [26]

$$
\frac{1}{2}\left(\frac{\partial \phi_{\mathrm{i}}}{\partial \xi}\right)^{2}+V_{\mathrm{S}}\left(\phi_{\mathrm{i}} ; M_{\mathrm{i}}, \tau, \beta_{\mathrm{i}}\right)=0,
$$

where the Sagdeev potential (with $\phi_{i, \max }=0$ ) is

$$
\begin{aligned}
V_{\mathrm{S}}\left(\phi_{\mathrm{i}} ; M_{\mathrm{i}}, \tau, \beta_{\mathrm{i}}\right)= & -\frac{1}{\tau}\left\{\frac{1}{\tau}\left[\exp \left(\tau \phi_{\mathrm{i}}\right)-1\right]\right. \\
& \left.+\exp \left(-\frac{M_{\mathrm{i}}^{2}}{2}\right)\left[P\left(-\phi_{\mathrm{i}}, \beta_{\mathrm{i}}\right)+h\left(\frac{M_{\mathrm{i}}^{2}}{2}, 0,-\phi_{\mathrm{i}}\right)-1\right]\right\} .
\end{aligned}
$$

Eq. (151), which is obtained from Eq. (147) in the limit of vanishing Langmuir wave electric fields, determines the profile of ion holes. The latter exist provided that $V_{\mathrm{S}}\left(\phi_{\mathrm{i}} ; M_{\mathrm{i}}, \tau, \beta_{\mathrm{i}}\right)$ is negative for $\phi_{\mathrm{i}}$ between zero and $-\psi$. The nonlinear dispersion relation $V_{\mathrm{S}}\left(-\psi_{\mathrm{i}} ; M_{\mathrm{i}}, \tau, \beta_{\mathrm{i}}\right)=0$ gives a relation between $\psi_{\mathrm{i}}$ and $M_{\mathrm{i}}$ for given values of $\beta_{\mathrm{i}}$ and $\tau$.

We have carried out numerical studies of the equations governing ion holes with and without Langmuir waves for $\tau=0.1$ and $\beta_{\mathrm{i}}=-1.0$. First, we consider small-amplitude Langmuir waves which are not strong enough to modify the ion hole, but which can be linearly trapped in the electron density well of the hole. Accordingly, for $W^{2} \ll 1 \mathrm{Eq}$. (144) turns into a linear eigenvalue problem of the form

$$
3 \frac{\mathrm{d}^{2} W}{\mathrm{~d} \xi^{2}}+\left[1-\exp \left(\tau \phi_{\mathrm{i}}\right)-\lambda\right] W=0,
$$

with the eigenvalue $\lambda$ and the corresponding eigenfunction $W$, and where $\phi_{\mathrm{i}}$ is obtained by assuming $W=0$ in the solution of Eq. (145); in the upper and lower panels of Fig. 34, we display the ion density profiles and the associated ambipolar potentials for different sets of parameters, where the obtained negative potentials are used in the eigenvalue problem (153). The eigenvalue problem (153) has a continuous spectrum for $\lambda<0$, associated with non-localized, propagating Langmuir wave envelopes $W$, and a point spectrum for $\lambda>0$, corresponding to localized, trapped Langmuir wave envelopes. We have investigated numerically the cases corresponding to four different Mach numbers displayed in Fig. 34, and found the corresponding positive eigenvalues listed in the left columns of Table 1, where each eigenvalue 

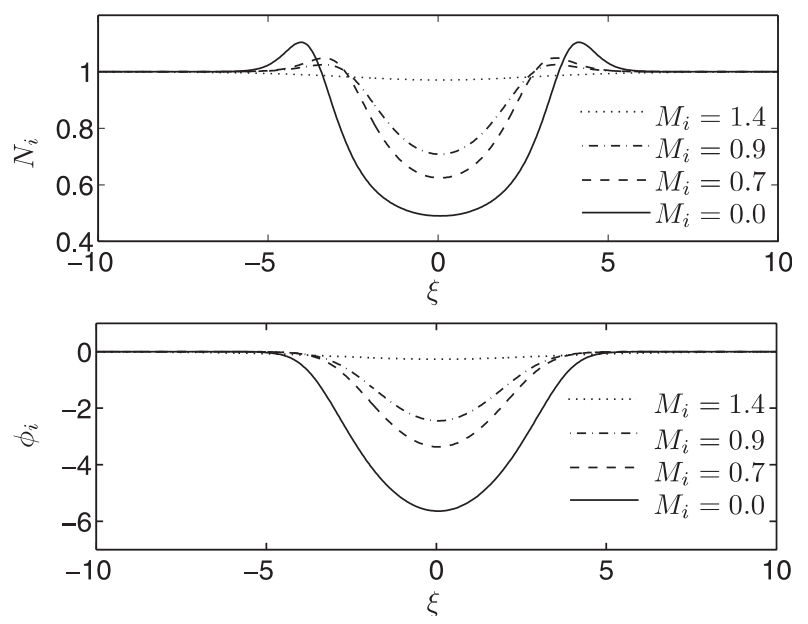

Fig. 34. The ion density (upper panel) and the potential (lower panel) of ion holes without Langmuir waves $(W=0)$ for different Mach numbers $M_{\mathrm{i}}$, with $\tau=0.1$ and $\beta_{\mathrm{i}}=-1.0$ (after Ref. [111]).

Table 1

Theoretical eigenvalues $\lambda$ and normalized frequencies $\omega / \omega_{\mathrm{pe}}$ for trapped Langmuir waves inside ion holes propagating with different Mach numbers $M_{\mathrm{i}}$

\begin{tabular}{|c|c|c|c|c|c|}
\hline \multirow[t]{2}{*}{$\begin{array}{l}\text { Mach } \\
\text { number, } M_{\mathrm{i}}\end{array}$} & \multicolumn{2}{|c|}{$\begin{array}{l}\text { Linear eigenvalues } \\
\text { and frequencies }\end{array}$} & \multirow{2}{*}{$\begin{array}{l}\text { Frequencies from } \\
\text { Vlasov simulation, } \\
\omega / \omega_{\mathrm{pe}}=\left(\omega_{\mathrm{r}}+\mathrm{i} \gamma\right) / \omega_{\mathrm{pe}}\end{array}$} & \multicolumn{2}{|c|}{$\begin{array}{l}\text { Nonlinear eigenvalues } \\
\text { and frequencies }\end{array}$} \\
\hline & $\lambda$ & $\omega / \omega_{\text {pe }}$ & & $\lambda$ & $\omega / \omega_{\mathrm{pe}}$ \\
\hline 1.4 & 0.0013 & 0.999 & & - & - \\
\hline 0.9 & 0.0463 & 0.977 & $0.98-0.00 \mathrm{i}$ & 0.1463 & 0.927 \\
\hline 0.7 & 0.0772 & 0.961 & & 0.1772 & 0.911 \\
\hline 0.0 & 0.1906 & 0.905 & $0.93-0.03 \mathrm{i}$ & 0.2906 & 0.855 \\
\hline
\end{tabular}

Theoretical linear values are compared with numerical values obtained from direct Vlasov simulation (left and middle columns). Eigenvalues (and frequencies) used in the nonlinear theoretical treatment of large-amplitude trapped Langmuir wave envelopes are shown in the right columns (after Ref. [111]).

$\lambda$ is associated to a bell-shaped eigenfunction $W$. Using $k_{0}=0$ in the definition of $\lambda$, we obtain the oscillation frequency of the trapped Langmuir waves as $\omega / \omega_{\mathrm{pe}}=1+\Theta=1-\lambda / 2+\left(u_{0} / V_{T e}\right)^{2} / 6=1-\lambda / 2+M_{\mathrm{i}}^{2} \tau m_{\mathrm{e}} / 6 m_{\mathrm{i}}$. Only one positive eigenvalue was found for each case, admitting only the ground states for the Langmuir waves to be linearly trapped.

Next, we examine the influence of finite-amplitude Langmuir waves on the ion hole, in which the fully nonlinear system of Eqs. (144) and (145) has to be solved numerically, and where we use the same parameters as in the linear treatment above. The numerical solutions reveal that the ion hole is deepened and widened, admitting the eigenvalue $\lambda$ to become larger. We have investigated the special case with a nonlinear shift of 0.1 of $\lambda$, listed in the column with nonlinear eigenvalues in Table 1 , and have found solutions for all cases except for $M_{\mathrm{i}}=1.4$; the numerical solutions are depicted in Fig. 35. Comparing with Fig. 34, we see that the presence of trapped finite-amplitude Langmuir waves makes the ion density depletion both deeper and wider, and the same holds for the ambipolar potential well. The deepening of the ambipolar negative potential well is a feature closely related to the strongly non-isothermal trapped ion distribution function. For this case, the electrostatic potential had small-amplitude maxima $\phi_{\mathrm{i}, \max }$ of the order $\approx 10^{-3}$ on each side of the ion hole, and this maximum of the potential increased with increasing $M_{\mathrm{i}}$.

Physically, the broadening of the ion hole and the enhancement of negative ambipolar potential occur because the ponderomotive force of the Langmuir waves locally expels electrons, which pull ions along to maintain the local charge neutrality. The deficit of the ions in plasmas, in turn, produces more negative potential within the ion hole that is now widened and enlarged to trap the localized Langmuir wave electric field envelope. 

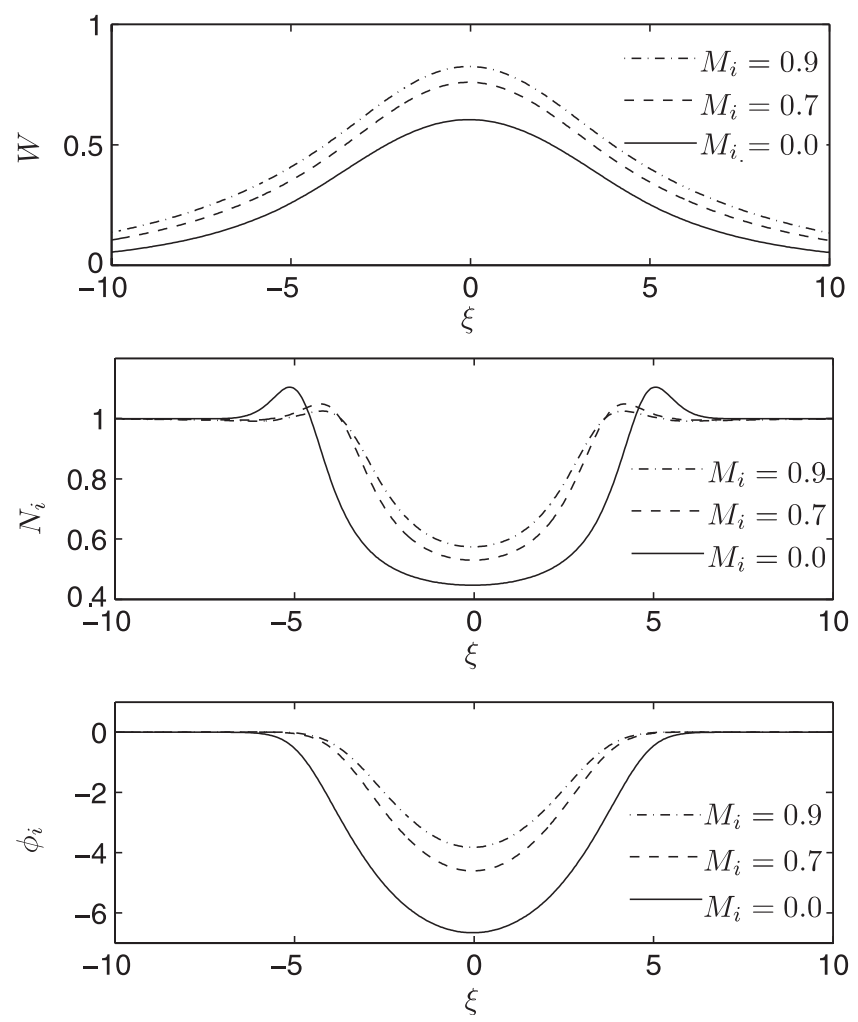

Fig. 35. The electric field (upper panel), the ion density (middle panel) and the potential (lower panel) of ion holes in the presence of large-amplitude Langmuir waves for different Mach numbers $M_{\mathrm{i}}$, with $\tau=0.1$ and $\beta_{\mathrm{i}}=-1.0$ (after Ref. [111]).

In order to investigate the conditions for the existence of ion holes in the presence of strong Langmuir fields, we numerically solved Eq. (150) for $\psi$ as a function of $M_{\mathrm{i}}$; see Fig. 36. We used the same parameters $\tau=0.1$ and $\beta_{\mathrm{i}}=-1.0$ as above. Here, we assumed the Langmuir field to be given as an external parameter (say $W_{0}=0.8$ ) and with a nonlinear shift that approximately follows the relation $\lambda\left(M_{\mathrm{i}}\right)=0.3-0.14 M_{\mathrm{i}}$ obtained from the fourth column of Table 1 . This relation overestimates slightly the Langmuir field $W_{0}$ for small $M_{\mathrm{i}}$ and underestimates slightly the field for the highest $M_{\mathrm{i}}$; see the upper panel in Fig. 35. We assumed a maximum potential of $\phi_{\mathrm{i}, \max }=0.003$. We found that for this set of parameters, the solution had an upper bound $M_{\mathrm{i}}=1.25$ for the existence of localized solutions, which is clearly smaller than the existence in the absence of the Langmuir fields. In a more exact mapping of the existence of ion holes, one needs to explore more carefully the relationships between different parameters in Eq. (150), possibly by solving the system of equations (144) and (145) for different cases. Furthermore, the dynamics of the time-dependent system is not explored here, but is studied by direct simulations of the Vlasov-Poisson system below.

It should be stressed that the properties of the present Langmuir envelope solitons significantly differ from those based on Zakharov's model [63] which utilizes the fluid ion response for driven (by the Langmuir wave ponderomotive force) ion-acoustic perturbations and yield subsonic density depression accompanied with a positive localized ambipolar potential structure. Furthermore, consideration of a Boltzmann ion density distribution, viz. $N_{\mathrm{i}}=\exp \left(-\phi_{\mathrm{i}}\right)$, would correspond to the case $M_{\mathrm{i}}=0$ and $\beta_{\mathrm{i}}=1$ in Eq. (145). Here, as shown in Fig. 37, we have a localized Langmuir wave electric field envelope trapped in a standing ion density cavity. The corresponding slow ambipolar potential is positive and localized.

In the numerical solutions of Eqs. (144) and (145), the second derivatives were approximated by a second-order centered difference scheme [157], and the values of $W$ and $\phi_{\mathrm{i}}$ were set to zero at the boundaries of the computational domain at $\xi= \pm 40$. The resulting nonlinear system of equations was solved iteratively. We used 2000 sampling points to resolve the solution. 


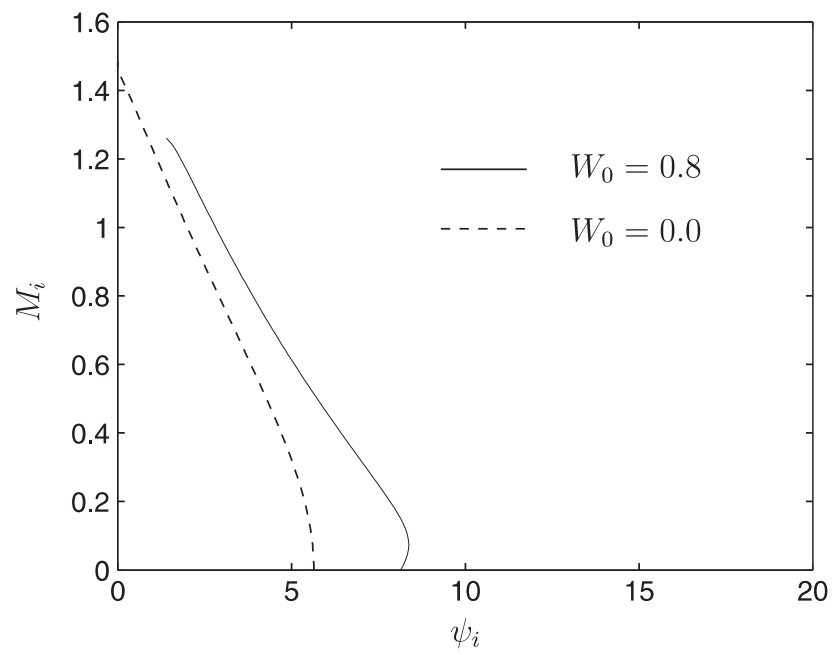

Fig. 36. Numerical solutions of Eq. (150), depicting $\psi\left(=-\phi_{\mathrm{i}, \min }\right)$ versus $M_{\mathrm{i}}$ for $W_{0}=0.0$ and $W_{0}=0.8$, with $\tau=0.1$ and $\beta_{\mathrm{i}}=-1.0$. We see that the ion hole loaded with the Langmuir wave electric fields has an upper bound on the Mach number, which is smaller than the one without the Langmuir wave fields (after Ref. [111]).
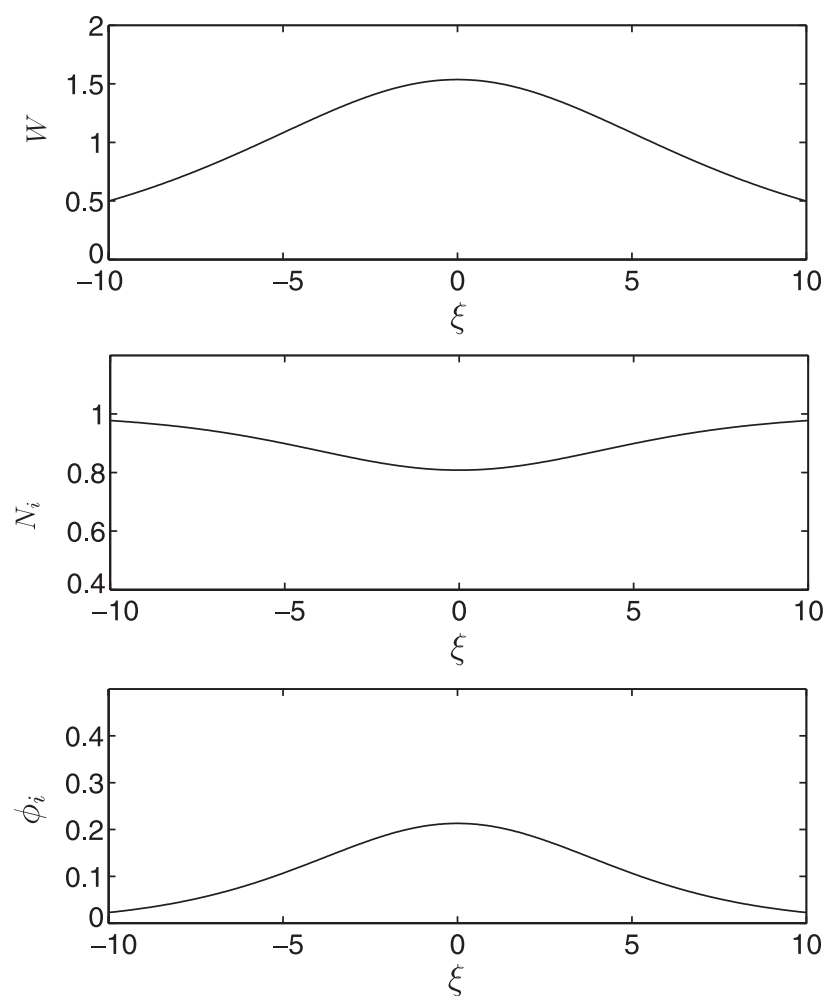

Fig. 37. The Langmuir electric field (upper panel), the ion density (middle panel) and the potential (lower panel) of a Langmuir caviton with a Boltzmann ion distribution for $M_{\mathrm{i}}=0, \lambda=0.1, \tau=0.1$ and $\beta_{\mathrm{i}}=1.0$ (after Ref. [111]).

In order to explore the dynamics of trapped Langmuir waves in an ion hole, we have performed a new set of simulations to study the time-dependence of trapped Langmuir wave electric fields. The results are displayed in Fig. 38. In these simulations, the electron density is initially perturbed so that Langmuir waves are excited around the ion hole centered 

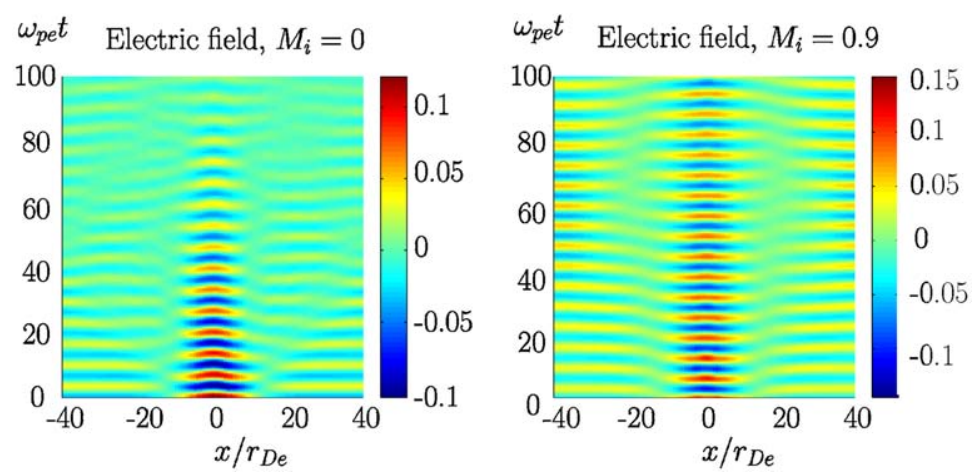

Fig. 38. Normalized Langmuir wave Electric field $E \sqrt{\epsilon_{0} / n_{0} k_{\mathrm{B}} T_{\mathrm{i}}}$ (the bipolar electric field has been removed from the data) as a function of the normalized space $x / r_{\text {De }}$ and time $\omega_{\text {pe }} t$. The Langmuir wave is trapped in the ion hole which is initially centered at $x / r_{\text {De }}=0$, and moving with the speed $M_{\mathrm{i}}=0$ (left panel) and $M_{\mathrm{i}}=0.9$ (right panel) (after Ref. [110]).

at $x / r_{\mathrm{De}}=0$. We examine the above-discussed ion hole with zero-speed (the left panel) and the ion hole having the speed $M_{\mathrm{i}}=0.9$ (the right panel). Fig. 38 shows that Langmuir waves are trapped in the ion hole, with a maximum wave amplitude at $x / r_{\mathrm{De}}=0$. We find that the trapped Langmuir waves are oscillating with a real frequency slightly lower than the electron plasma frequency, $\omega_{\mathrm{r}} / \omega_{\mathrm{pe}} \approx 0.93$ for the standing ion hole case, and $\omega_{\mathrm{r}} / \omega_{\mathrm{pe}} \approx 0.98$ for the moving ion hole case with $M_{\mathrm{i}}=0.9$, which is consistent with the linear results listed in Table 1 . For the standing hole case, the Langmuir waves are strongly Landau damped, with a damping rate $\gamma \approx 0.03 \omega_{\text {pe }}$, which is an effect not covered by our theoretical model which is based on the electron hydrodynamic model for the Langmuir wave packets. In the moving ion hole case, the damping is significantly weaker than in the standing hole case. This can be understood in that the frequency shift is smaller for the moving ion hole case, making the scale length larger for the Langmuir wave envelope, resulting in a smaller Landau damping; some distance away from the ion hole density minimum the potential $\phi_{\mathrm{i}}$ vanishes, and there the Langmuir wave envelope decreases exponentially with $\xi$ with the scale length $\sqrt{3 / \lambda}$. A smaller $\lambda$ leads to a larger length-scale, resulting in a weaker Landau damping. In numerical simulations of large-amplitude Langmuir waves, one has to apply an external pump field to sustain the Langmuir field at constant amplitude, since the trapped Langmuir waves are Landau damped. We have observed the following phenomena in Vlasov simulations: High-intensity Langmuir waves are initially trapped inside the ion hole. The ion density well associated with the ion hole deepened, and the ion density surrounding the ion hole is depleted. The Langmuir field trapped in the ion hole accelerated electrons which formed propagating electron BGK waves (electron holes). We could not observe a clear widening of the ion hole predicted by theory. Possible explanations of this discrepancy can be that the electrons are strongly heated in the simulation, which increases the temperature ratio $\tau$, leading to a scenario in which the ion hole tends to become smaller if other parameters are kept constant [26]. Further, the theory does not predict how the parameters of an ion hole changes dynamically when the amplitude of the trapped Langmuir envelope changes in time.

\subsection{The influence of high-Z impurities (dust) on ion holes}

The results of the preceding section have been generalized to include the influence of high- $Z$ impurities (or charged micron-sized dust) which occur in laboratory and space plasmas [154]. Our numerical results reveal new features of the Langmuir envelope solitons in electron-ion-dust plasmas containing stationary and mobile charged dust grains. The presence of a relatively small amount of dust makes the ion hole decrease drastically in size. Dusty plasmas typically contain micron-sized dust grains with $Z_{\mathrm{d}}=10^{3}$, where $Z_{\mathrm{d}}$ is the number of charges residing on the dust grain surface, and the dust grain to ion mass ratio $m_{\mathrm{d}} / m_{\mathrm{i}}=10^{12}$. The dust grains are charged negatively or positively depending on the plasma environments [158-163]. The ion to electron mass ratio is 1836 or more depending on the ion species. In laboratory dusty plasmas, we typically have the electron to ion temperature ratio $T_{\mathrm{e}} / T_{\mathrm{i}}=10$ and the electron temperature $k_{\mathrm{B}} T_{\mathrm{e}} \sim 10 \mathrm{eV}$. 
We consider a fully ionized dusty plasma composed of electrons, ions, and charged dust particles. At equilibrium, we have

$$
n_{\mathrm{e} 0}=n_{\mathrm{i} 0}-\epsilon Z_{\mathrm{d}} n_{\mathrm{d} 0},
$$

where $n_{j 0}$ is the unperturbed number density of the particle species $j$ ( $j$ equals e for electrons, $i$ for ions and $\mathrm{d}$ for dust particles), $\epsilon=+1(-1)$ for negatively (positively) charged dust, and $Z_{\mathrm{d}}$ is the number of charges residing on the dust grain surface.

The slowly varying electron and ion number densities are given by Eqs. (140) and (141), respectively, and the potential is given by Poisson's equation

$$
\tau r_{\text {De }}^{2} \frac{\partial^{2} \phi_{\mathrm{i}}}{\partial x^{2}}=N_{\mathrm{e}}-\alpha N_{\mathrm{i}}+\alpha-1
$$

where $\alpha=n_{\mathrm{i} 0} / n_{\mathrm{e} 0}$. We assume the dust to be stationary so that $Z_{\mathrm{d}} N_{\mathrm{d}}=Z_{\mathrm{d}} n_{\mathrm{d} 0}$.

In a frame moving with constant speed $u_{\mathrm{i} 0}$, Poisson's equation (155) for the IH perturbation is

$$
\begin{aligned}
\tau \frac{\partial^{2} \phi_{\mathrm{i}}}{\partial \xi^{2}}= & \exp \left(\tau \phi_{\mathrm{i}}-W^{2}\right)-\alpha b \exp \left(-\frac{M_{\mathrm{i}}^{2}}{2}\right) \\
& \times\left[I\left(-\Phi_{\mathrm{i}}\right)+\kappa\left(\frac{M_{\mathrm{i}}^{2}}{2},-\Phi_{\mathrm{i}}\right)+\frac{2}{\sqrt{\pi\left|\beta_{\mathrm{i}}\right|}} W_{\mathrm{D}}\left(\sqrt{\beta_{\mathrm{i}} \Phi_{\mathrm{i}}}\right)\right]+\alpha-1,
\end{aligned}
$$

where $M_{\mathrm{i}}=u_{\mathrm{i} 0} / V_{T i}$, and $W$ is obtained from the solution of Eq. (144).

Eqs. (144) and (145) are the desired equations for the investigation of cavitons in an unmagnetized dusty plasma. They admit the conserved quantity (Hamiltonian)

$$
H\left(W, \phi_{\mathrm{i}}, \lambda, M_{\mathrm{i}}, \tau, \beta_{\mathrm{i}}, \alpha\right)=3\left(\frac{\partial W}{\partial \xi}\right)^{2}-\frac{\tau}{2}\left(\frac{\partial \phi_{\mathrm{i}}}{\partial \xi}\right)^{2}+V\left(\phi_{\mathrm{i}}, W, M_{\mathrm{i}}, \beta_{\mathrm{i}}, \tau, \alpha\right)=0,
$$

where we have the "potential energy"

$$
\begin{aligned}
V\left(\phi_{\mathrm{i}}, W, M_{\mathrm{i}}, \beta_{\mathrm{i}}, \tau, \alpha\right)= & (1-\lambda) W^{2}+\frac{1}{\tau}\left\{\exp \left[\tau\left(\phi_{\mathrm{i}}-W^{2}\right)-1\right]\right\} \\
& +\alpha b \exp \left(-\frac{M_{\mathrm{i}}^{2}}{2}\right)\left[P\left(-\Phi_{\mathrm{i}}, \beta_{\mathrm{i}}\right)-P\left(\phi_{\mathrm{i}, \max }, \beta_{\mathrm{i}}\right)\right. \\
& \left.+h\left(\frac{M_{\mathrm{i}}^{2}}{2}, 0,-\Phi_{\mathrm{i}}\right)-h\left(\frac{M_{\mathrm{i}}^{2}}{2}, 0, \phi_{\mathrm{i}, \max }\right)\right]+(\alpha-1) \phi_{\mathrm{i}} .
\end{aligned}
$$

The auxiliary functions $P$ and $h$ are defined in Eqs. (148) and (149), respectively.

Since we are interested in symmetric solutions defined by $W^{2}(\xi)=W^{2}(-\xi)$ and $\phi_{\mathrm{i}}(\xi)=\phi_{\mathrm{i}}(-\xi)$, we have the unperturbed state $(|\xi|=\infty)$ used the appropriate boundary conditions $W=0, \phi_{\mathrm{i}}=0, \partial W / \partial \xi=0$ and $\partial \phi_{\mathrm{i}} / \partial \xi=0$. On the other hand, the boundary conditions at $\xi=0$ are $W=W_{0}, \phi_{\mathrm{i}}=\phi_{\mathrm{i} 0}, \partial W / \partial \xi=0$ and $\partial \phi_{\mathrm{i}} / \partial \xi=0$. The relation $V\left(\phi_{\mathrm{i} 0}, \phi_{\mathrm{i}, \max }, W_{0}, M_{\mathrm{i}}, \beta_{\mathrm{i}}, \tau, \alpha\right)=0$ constitutes a relation between the potential and Langmuir wave envelopes for given parameters.

In the absence of the Langmuir waves (viz. $W=0$ ), we obtain from (157)-(158) the energy integral

$$
\frac{1}{2}\left(\frac{\partial \phi_{\mathrm{i}}}{\partial \xi}\right)^{2}+V_{\mathrm{S}}\left(\phi_{\mathrm{i}} ; M_{\mathrm{i}}, \beta_{\mathrm{i}}, \tau, \alpha\right)=0
$$

where the Sagdeev potential is (with $\phi_{\mathrm{i}, \max }=0$ )

$$
\begin{aligned}
V_{\mathrm{S}}\left(\phi_{\mathrm{i}} ; M_{\mathrm{i}}, \beta_{\mathrm{i}}, \tau, \alpha\right)= & -\frac{1}{\tau}\left\{\frac{1}{\tau}\left[\exp \left(\tau \phi_{\mathrm{i}}\right)-1\right]\right. \\
& \left.+\alpha \exp \left(-\frac{M_{\mathrm{i}}^{2}}{2}\right)\left[P\left(-\phi_{\mathrm{i}}, \beta_{\mathrm{i}}\right)+h\left(\frac{M_{\mathrm{i}}^{2}}{2}, 0,-\phi_{\mathrm{i}}\right)-1\right]-(\alpha-1) \phi_{\mathrm{i}}\right\} .
\end{aligned}
$$

In the absence of dust, viz. $\alpha=1$, we recover exactly from Eq. (160) the previous results in Eq. (152). 

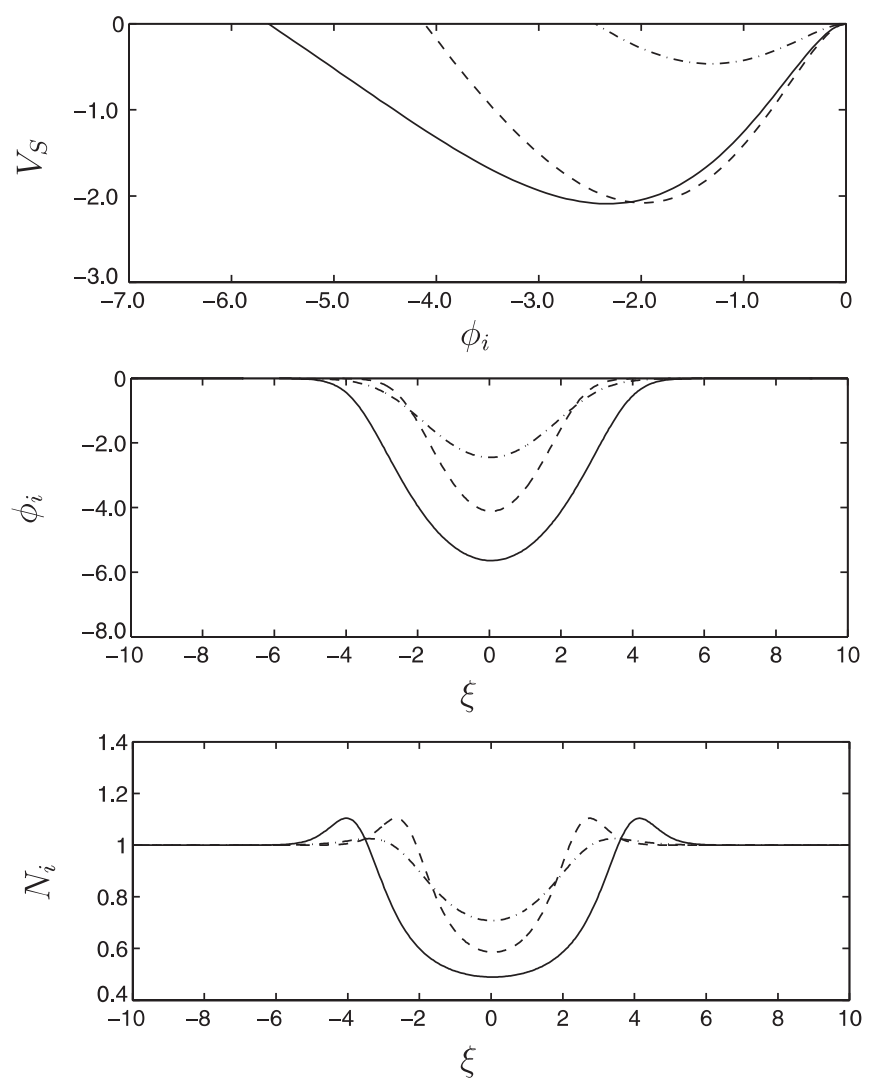

Fig. 39. The Sagdeev potential (upper panel), electrostatic potential (middle panel), and ion density (lower panel) associated with an ion hole without Langmuir waves $\left(W=0\right.$ ). Parameters are $M_{\mathrm{i}}=0$, and $\alpha=1.0$ (solid line), $M_{\mathrm{i}}=0$ and $\alpha=1.2$ (dashed line), and $M_{\mathrm{i}}=0.9$ and $\alpha=1.0$ (dash-dotted line). We used $\tau=0.1$ and $\beta_{\mathrm{i}}=-1.0$ in all cases (after Ref. [154]).

In the absence of Langmuir waves, ion holes are associated with negative localized electrostatic potentials. This will lead to localized electron density cavities by Eq. (140) (with $W=0$ ), which can work as a resonance cavity which linearly trap Langmuir waves. Accordingly, for $W^{2} \ll 1$, Eq. (144) turns into a linear eigenvalue problem in the form

$$
3 \frac{\partial^{2} W}{\partial \xi^{2}}+\left[1-\exp \left(\tau \phi_{\mathrm{i}}\right)-\lambda\right] W=0,
$$

with the eigenvalue $\lambda$ and the corresponding eigenfunction $W$, and with the boundary conditions $W=0$ at $|\xi|=\infty$. Here, $\phi_{\mathrm{i}}$ is obtained by assuming $W^{2}=0$ in the solutions of Eq. (156). The eigenvalue problem (161) will have a discrete set of positive eigenvalues associated with localized eigenfunctions, and a continuous set of negative eigenvalues associated with non-localized eigenfunctions. For the symmetric ion holes studied here, the largest positive eigenvalue $\lambda_{1}$ will be associated with an even (with respect to $\xi$ ) eigenfunction, the next largest eigenvalue $\lambda_{2}$ will be associated with an odd eigenfunction, etc. If the electric field envelope $W$ has a large amplitude, then the ponderomotive force acting on the electrons will modify the electron density [by Eq. (140)] which will modify the electrostatic potential via Poisson's equation, and as a result Eq. (144) will be nonlinearly coupled to Eq. (145), leading to a nonlinear eigenvalue problem.

We here focus on ion holes which are associated with a vortex-like ion distribution function and a self-consistent negative electrostatic potential. The negative potential well leads to a cavity which can trap Langmuir waves. In Fig. 39, we have studied examples of ion holes and the influence of dust $(\alpha=1.2)$ on one standing ion hole and one moving ion hole $\left(M_{\mathrm{i}}=0.9\right)$ in the absence of dust. The presence of the relatively small amount of dust has a large effect on the ion hole, whose potential well and density cavity becomes both narrower and shallower; see the middle and lower panel of 
Table 2

Frequency shifts for Langmuir waves trapped in IH waves; small-amplitude Langmuir waves (left) and an example with large-amplitude Langmuir waves (right) (after Ref. [154])

Small-amplitude problem

\begin{tabular}{|c|c|c|c|c|c|}
\hline$M_{\mathrm{i}}=0.0$ & $\alpha=1.0$ & $\lambda_{1}=0.1906$ & $M_{\mathrm{i}}=0.0$ & $\alpha=1.0$ & $\lambda_{1}=0.290$ \\
\hline$M_{\mathrm{i}}=0.0$ & $\alpha=1.2$ & $\lambda_{1}=0.0792$ & $M_{\mathrm{i}}=0.0$ & $\alpha=1.2$ & $\lambda_{1}=0.290$ \\
\hline$M_{\mathrm{i}}=0.9$ & $\alpha=1.0$ & $\lambda_{1}=0.0466$ & $M_{\mathrm{i}}=0.9$ & $\alpha=1.0$ & $\lambda_{1}=0.146$ \\
\hline
\end{tabular}
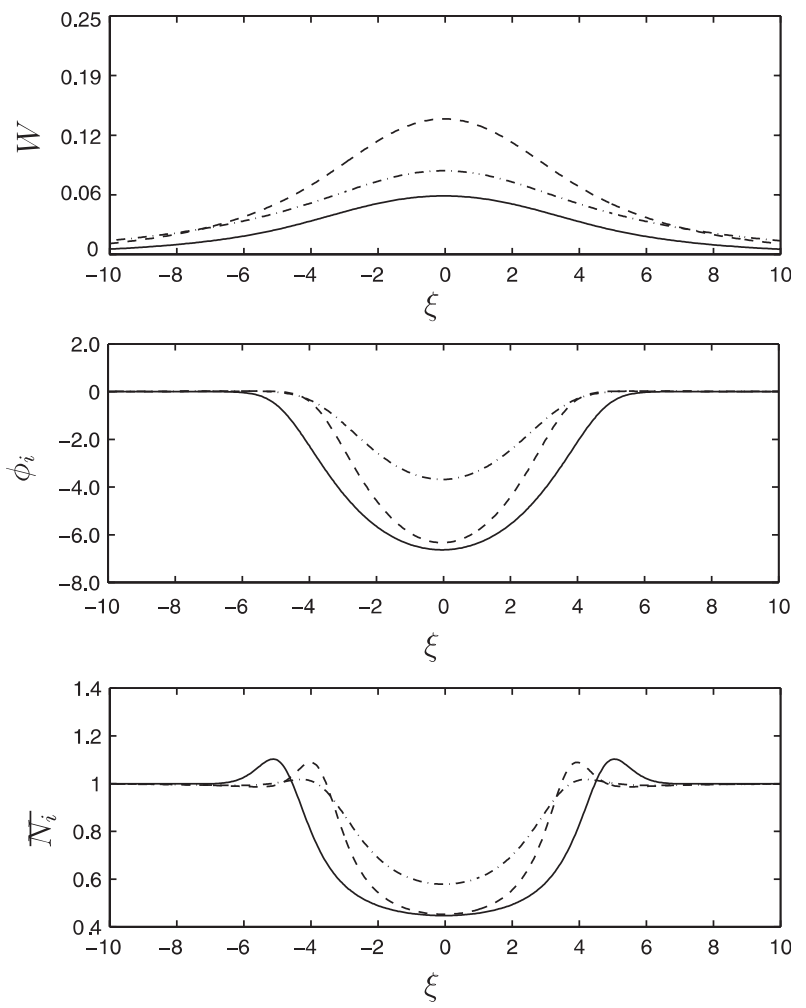

Fig. 40. The Langmuir wave electric field amplitude (upper panel), electrostatic potential (middle panel), and ion density (lower panel) associated with an ion hole in the presence of Langmuir waves. Parameters are $M_{\mathrm{i}}=0, \alpha=1.0$ and $\lambda=0.290$ (solid line), for $M_{\mathrm{i}}=0$ and $\alpha=1.2$ and $\lambda=0.290$ (dashed line) and for $M_{\mathrm{i}}=0.9$ and $\alpha=1.0$ and $\lambda=0.146$ (dash-dotted line). We used $\tau=0.1$ and $\beta_{\mathrm{i}}=-1.0$ in all cases (after Ref. [154]).

Fig. 39. The moving ion hole without dust becomes much shallower and also somewhat narrower than the standing ion hole without dust. We next studied the linear trapping of Langmuir waves in the ion holes, with the same parameters as in Fig. 39. Accordingly, we insert the potentials into Eq. (161) and solve the linear eigenvalue problems for $\lambda$ and $W$. For each of the three cases, we could only find one positive eigenvalue $\lambda_{1}$, listed in the left column of Table 2 . Each of these eigenvalues were associated with a symmetric, bell-shaped eigenfunction $W_{1}$. In the presence of high-amplitude Langmuir waves (see Fig. 40), the ion hole in general becomes wider and deeper, allowing the positive eigenvalues to become larger; see the right columns of Table 2. The deepening of the ambipolar negative potential well is a feature closely related to the strongly non-isothermal trapped ion distribution function. For this case, the electrostatic potential had small-amplitude maxima $\phi_{i, \max }>0$ of the order $\approx 10^{-3}$ on each side of the ion hole, this maximum of the potential was larger for the case with larger $M_{\mathrm{s}}$.

In the numerical solutions of Eqs. (144) and (145), the second derivatives were approximated by a second-order centered scheme [157], and the values of $W$ and $\varphi$ were set to zero at the boundaries of the computational domain. The resulting nonlinear system of equations was solved with Newton's method. We used 2000 sampling points to resolve the solution. 


\subsection{Trapping of intense electromagnetic waves by REHs}

Intense electromagnetic waves are common in astrophysical settings as well as in inertial confinement fusion schemes that contain extremely hot plasmas. In the latter, one may encounter efficient nonlinear interactions between radiation and the background plasma. In what follows, we present fully relativistic nonlinear theory and computer simulations of intense localized electromagnetic that are trapped in REHs in a relativistically hot electron plasma [155]. We adopt the Maxwell-Poisson-relativistic Vlasov system, which accounts for relativistic electron mass increase in the electromagnetic fields and the relativistic radiation ponderomotive force, in addition to trapped electrons which support the driven REHs, and show how the latter trap intense laser light. The present complex nonlinear interactions between intense electromagnetic waves and REHs produce intense electromagnetic envelope solitons composed of localized light wave envelope and large amplitude localized positive electric potential distributions. We also display numerical studies of the dynamics of interacting electromagnetic envelope solitons by means of a fully relativistic Vlasov simulations. Interesting nonlinear features of laser light intensification and corresponding density cavitation have been observed.

Let us consider the relevant equations describing the action of intense laser light on the electrons in a relativistically hot collisionless plasma, as well as the electromagnetic wave equation accounting for the relativistic mass increase of the electrons and the electron density modification due to the radiation relativistic ponderomotive force [80]

$$
F=-m_{\mathrm{e}} c^{2} \frac{\partial \gamma}{\partial z}
$$

where

$$
\gamma=\left(1+\frac{p^{2}}{m_{\mathrm{e}}^{2} c^{2}}+\frac{\mathrm{e}^{2}|\mathbf{A}|^{2}}{m_{\mathrm{e}}^{2} c^{4}}\right)^{1 / 2}
$$

is the relativistic gamma factor. Here, $p$ is the $x$ component of the electron momentum, and we have used that the perpendicular (to the $x$ direction) momentum $p_{\perp}=e \mathbf{A} / c$, where $\mathbf{A}$ is the vector potential of the circularly polarized electromagnetic waves. The dynamics of the coupled electromagnetic waves and relativistic electron holes with immobile ions is governed by

$$
\begin{aligned}
& \frac{\partial^{2} \mathbf{A}}{\partial t^{2}}-\frac{1}{\mu^{2}} \frac{\partial^{2} \mathbf{A}}{\partial x^{2}}+\int_{-\infty}^{\infty} \frac{f_{\mathrm{e}}}{\gamma} \mathrm{d} p \mathbf{A}=0, \\
& \frac{\partial f_{\mathrm{e}}}{\partial t}+\frac{p}{\gamma} \frac{\partial f_{\mathrm{e}}}{\partial x}+\frac{\partial\left(\phi-\gamma / \mu^{2}\right)}{\partial x} \frac{\partial f_{\mathrm{e}}}{\partial p}=0
\end{aligned}
$$

and

$$
\frac{\partial^{2} \phi}{\partial x^{2}}=\int_{-\infty}^{\infty} f_{\mathrm{e}} \mathrm{d} p-1
$$

where $\mathbf{A}$ is scaled by $m_{\mathrm{e}} c / e$ and the other quantities are normalized the same way as in Section 4.1. We have denoted $\gamma=\left(1+\mu^{2} p^{2}+|\mathbf{A}|^{2}\right)$.

Far away from the relativistic electron hole, where $\phi=|\mathbf{A}|=0$, the electrons are assumed to obey a Jüttner-Synge distribution function [130]

$$
\tilde{f}_{0}(p)=a_{0} \exp \left[-\frac{1}{\mu^{2}}\left(\sqrt{1+\mu^{2} p^{2}}-1\right)\right],
$$

where the normalization constant $a_{0}$ is given by Eq. (72). For $f_{\mathrm{e}}=\tilde{f}_{0}(p)$, the last term in the left-hand side of Eq. (164) takes the value

$$
\int_{-\infty}^{\infty} \frac{\tilde{f}_{0}(p)}{\sqrt{1+\mu^{2} p^{2}}}=\frac{K_{0}\left(\mu^{-2}\right)}{K_{1}\left(\mu^{-2}\right)} \equiv \Omega_{p}^{2},
$$

where $K_{0}$ and $K_{1}$ are the modified Bessel functions of second kind. The frequency $\Omega_{p}$ represents the normalized (by $\left.\omega_{\text {pe }}\right)$ relativistic plasma frequency at equilibrium. 
The properties of driven relativistic electron holes can be investigated as follows. We use the ansatz $f(p, \xi)$ for the relativistic electron distribution function, and assume that $\phi$ and $|A|^{2}$ depend on $\xi$ only, where $\xi=x-M_{\mathrm{e}} t$. Then, Eqs. (165) and (166) take the form

$$
\left(-M_{\mathrm{e}}+\frac{p}{\gamma}\right) \frac{\partial f_{\mathrm{e}}}{\partial \xi}+\frac{\partial\left(\phi-\gamma / \mu^{2}\right)}{\partial \xi} \frac{\partial f_{\mathrm{e}}}{\partial p}=0
$$

and

$$
\frac{\mathrm{d}^{2} \phi}{\mathrm{d} \xi^{2}}=\int_{-\infty}^{\infty} f_{\mathrm{e}} \mathrm{d} p-1
$$

respectively. The general solution of Eq. (169) is $f_{\mathrm{e}}=f_{0}(\mathscr{E})$, where $f_{0}$ is some function of one variable and

$$
\mathscr{E}=-M_{\mathrm{e}} p+\frac{1}{\mu^{2}}\left(\gamma-\frac{1}{\gamma_{0}}\right)-\phi
$$

is the energy integral. Here, we have denoted $\gamma_{0}=1 / \sqrt{1-\mu^{2} M_{\mathrm{e}}^{2}}$. We note that trapped electrons have negative energy while untrapped (free) electrons have positive energy.

In in the slowly varying envelope approximation, viz. $\mathbf{A}=(1 / 2) A(x, t)(\hat{\mathbf{y}}+\mathrm{i} \hat{\mathbf{z}}) \exp \left(-\mathrm{i} \omega_{0} t+\mathrm{i} k_{0} x\right)+$ complex conjugate, Eq. (164) can be written as

$$
2 \mathrm{i} \omega_{0}\left(\frac{\partial A}{\partial t}+v_{\mathrm{g}} \frac{\partial A}{\partial x}\right)+\frac{1}{\mu^{2}} \frac{\partial^{2} A}{\partial x^{2}}-\left(\int_{-\infty}^{\infty} \frac{f_{\mathrm{e}}}{\gamma} \mathrm{d} p-\Omega_{p}^{2}\right) A=0,
$$

where $\omega_{0}=\left(\Omega_{p}^{2}+k_{0}^{2} / \mu^{2}\right)^{1 / 2}$ is the electromagnetic wave frequency, $v_{\mathrm{g}}=k_{0} / \mu^{2} \omega_{0}$ is the group velocity, and $\gamma=\sqrt{1+\mu^{2} p^{2}+|A|^{2}}$. Introducing $A=W(\xi) \exp (-\mathrm{i} \Theta t+\mathrm{i} K x)$ into Eq. (172), where $W$ is a real-valued function, we obtain $K=\mu^{2} \omega_{0}\left(M_{\mathrm{e}}-v_{\mathrm{g}}\right)$ and

$$
\frac{\mathrm{d}^{2} W}{\mathrm{~d} \xi^{2}}-\lambda W-\mu^{2}\left(\Omega^{2}-\Omega_{p}^{2}\right) W=0
$$

where $\lambda=-2 \omega_{0} \mu^{2} \Theta+\mu^{4} \omega_{0}^{2}\left(M_{\mathrm{e}}^{2}-v_{\mathrm{g}}^{2}\right)$ represents a nonlinear frequency shift, and the gamma factor becomes $\gamma=\sqrt{1+\mu^{2} p^{2}+W^{2}}$. Here, $\Omega^{2}=\int_{-\infty}^{\infty}\left(f_{\mathrm{e}} / \gamma\right) \mathrm{d} p$ represents the square of the local electron plasma frequency that accounts for the relativistic electron mass increase in the electron hole potential and the electromagnetic wave fields.

A condition for the untrapped electron is that far away from the relativistic electron hole, the electron distribution function should smoothly connect to the Jüttner-Synge distribution function. In order to impose this condition for the free electrons, we use the solution

$$
f_{\mathrm{e}}=f_{0}(\mathscr{E})=\tilde{f}_{0}[\tilde{p}(\mathscr{E})], \quad \mathscr{E}>0
$$

where $\tilde{p}(\mathscr{E})$ is a function of the energy such that $\tilde{p}(\mathscr{E}) \rightarrow p$ when $W \rightarrow 0$ and $\phi \rightarrow 0$. Such a function can be found with the help of the energy integral by setting

$$
-M_{\mathrm{e}} \tilde{p}(\mathscr{E})+\frac{1}{\mu^{2}}\left(\sqrt{1+\mu^{2} \tilde{p}^{2}(\mathscr{E})}-\frac{1}{\gamma_{0}}\right)=\mathscr{E} .
$$

Solving for $\tilde{p}(\mathscr{E})$, we have

$$
\tilde{p}_{ \pm}(\mathscr{E})=\gamma_{0}^{2} M_{\mathrm{e}}\left(\mu^{2} \mathscr{E}+\frac{1}{\gamma_{0}}\right) \pm \frac{\gamma_{0}^{2}}{\mu} \sqrt{\left(\mu^{2} \mathscr{E}+\frac{1}{\gamma_{0}}\right)^{2}-\frac{1}{\gamma_{0}^{2}}}
$$


where $\tilde{p}_{+}(\mathscr{E})$ and $\tilde{p}_{-}(\mathscr{E})$ correspond to a modified momentum for free electrons on each side of the trapped electron population in momentum space. Using $\tilde{p}(\mathscr{E})=\tilde{p}_{+}(\mathscr{E})$ and $\tilde{p}(\mathscr{E})=\tilde{p}_{-}(\mathscr{E})$ in Eq. (174), we obtain the distribution function for free electrons. In the limit of vanishing energy, $\mathscr{E}=0^{+}$, we have $\tilde{p}_{ \pm}(0)=\gamma_{0} M_{\mathrm{e}}$, and the value of the distribution function is

$$
\left.f_{\mathrm{e}}\right|_{\mathscr{E}=0^{+}}=f_{0}(0)=\tilde{f}_{0}\left(\gamma_{0} M_{\mathrm{e}}\right)=a_{0} \exp \left[-\left(\gamma_{0}-1\right) / \mu^{2}\right]
$$

which should be matched with the distribution function for the trapped electrons with $\mathscr{E}=0$ in order to obtain a continuous distribution function. For the trapped electrons, we choose a relativistic Maxwell-Boltzmann distribution with a negative "temperature," viz.

$$
f_{\mathrm{e}}=a_{0} \exp \left(-\frac{\gamma_{0}-1}{\mu^{2}}-\beta_{\mathrm{e}} \mathscr{E}\right), \quad \mathscr{E}<0
$$

The separatrix between the free and trapped electron distributions is found where $\mathscr{E}=0$ in Eq. (171); solving for $p$, we then have

$$
p_{ \pm}=\gamma_{0}^{2} M_{\mathrm{e}}\left(\mu^{2} \phi+\frac{1}{\gamma_{0}}\right) \pm \frac{\gamma_{0}^{2}}{\mu} \sqrt{\left(\mu^{2} \phi+\frac{1}{\gamma_{0}}\right)^{2}-\frac{1+W^{2}}{\gamma_{0}^{2}}}
$$

where $p_{-}$and $p_{+}$constitute the limits between the trapped and free electron distributions in momentum space. Using these limits and combining Eqs. (174), (176) and (178), we can now write

$$
f_{\mathrm{e}}= \begin{cases}a_{0} \exp \left[-\frac{1}{\mu^{2}}\left(\sqrt{1+\mu^{2} \tilde{p}_{+}^{2}(\mathscr{E})}-1\right)\right], & p>p_{+}, \\ a_{0} \exp \left[-\frac{\gamma_{0}-1}{\mu^{2}}-\beta_{\mathrm{e}} \mathscr{E}\right], & p_{-} \leqslant p \leqslant p_{+} \\ a_{0} \exp \left[-\frac{1}{\mu^{2}}\left(\sqrt{1+\mu^{2} \tilde{p}_{-}^{2}(\mathscr{E})}-1\right)\right], & p<p_{-} .\end{cases}
$$

Integrating the distribution function (180) over the momentum space, we obtain the total electron number density as a function of $\phi$ and $W$, which are calculated self-consistently by means of the Poisson and Schrödinger equations (170) and (173), respectively.

Fig. 41 exhibits the influence of intense electromagnetic waves on relativistic electron holes, described by the coupled system of equations (170) and (173). In the Schrödinger equation (173), $\lambda$ represents the eigenvalue, and the square of the local electron plasma frequency, $\Omega^{2}$, enters as a "potential." We see that for larger electromagnetic fields $W$, the relativistic electron hole potential $\phi$ becomes larger and the relativistic electron hole wider, admitting larger eigenvalues $\lambda$. This can be explained in that the relativistic ponderomotive force of localized electromagnetic waves pushes the electrons away from the center of the relativistic electron hole, leading to an increase of the electrostatic potential distribution and a widening of the relativistic electron hole. We see that the depletion of the electron density in the relativistic electron hole is only minimal, while the local electron plasma frequency $\Omega$ is strongly reduced owing to the increased mass of the electrons that are accelerated by the relativistic electron hole potential. The linear trapping of electromagnetic waves in relativistic electron holes is displayed in Fig. 42, where we have assumed a zero electromagnetic field $(W=0)$ in the expression for $\gamma$ used in Eq. (173) and in the energy integral. The eigenvalue problem admits a discrete set of localized eigenfunctions with positive eigenvalues, and in this case we found two even and one odd eigenfunction corresponding to three different eigenvalues. Eq. (173) has been solved as a linear eigenvalue problem for $W$, where the amplitude $W_{\max }$ of the electromagnetic waves was kept fixed, to obtain new values on $W$ and $\lambda$. Then, the procedure of solving for $\phi$ and $W$ was repeated until convergence. The second derivatives were approximated with a second-order centered scheme with the function values set to zero at the boundaries. 

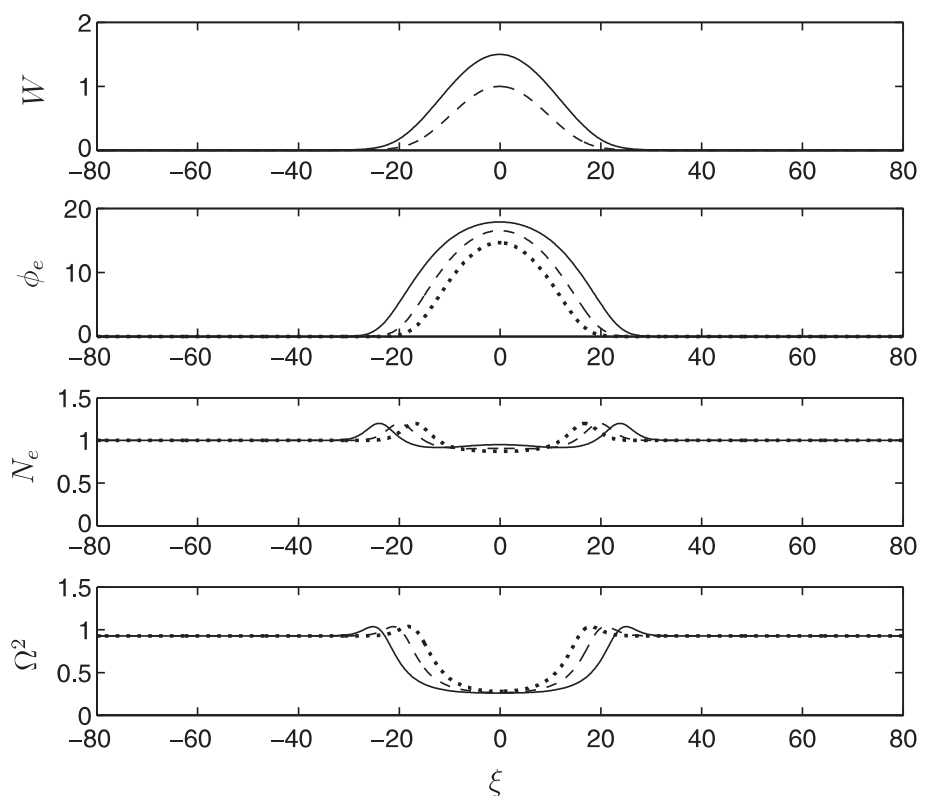

Fig. 41. Large-amplitude trapped electromagnetic wave envelope (upper panel), the potential (second panel), the electron number density (third panel), and the square of the local electron plasma frequency (lower panel) for large amplitude electromagnetic waves with a maximum amplitude of $W_{\max }=1.5$ (solid lines) and $W_{\max }=1.0$ (dashed lines), and as a comparison a relativistic electron hole with small-amplitude electromagnetic waves which have $W_{\max } \ll 1$ (dotted lines). The nonlinear frequency shift for the $W_{\max }=1.5$ case is $\lambda=0.099$, and for the $W_{\max }=1.0$ case it is $\lambda=0.095$, to be compared with the small-amplitude case which has $\lambda=0.088$. Parameters are: $M_{\mathrm{e}}=0.7, \mu=0.4$, and $\beta_{\mathrm{e}}=-0.5$ (after Ref. [155]).

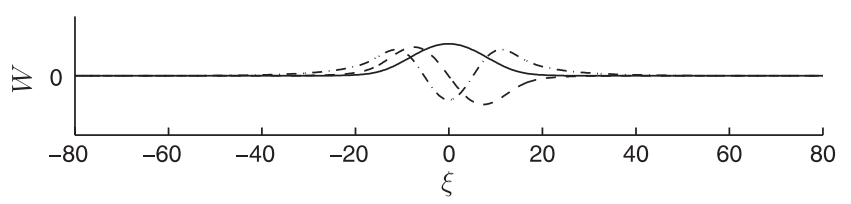

Fig. 42. Small-amplitude trapped electromagnetic waves (given in arbitrary units) in a relativistic electron hole. Three eigenstates of trapped electromagnetic waves, corresponding to the eigenvalues $\lambda_{1}=0.088$ (solid line), $\lambda_{2}=0.053$ (dashed line) and $\lambda_{2}=0.013$ (dash-dotted line). The parameters used are the same as for the dotted lines in Fig. 41 (after Ref. [155]).

In order to study the dynamics of interacting electromagnetic envelope solitons composed of localized electromagnetic waves and relativistic electron holes, we have solved the time-dependent, relativistic Vlasov equation (165) together with the Schrödinger equation (172) numerically. The results are displayed in Figs. 43 and 44. As an initialcondition to the simulation, we used solutions to the quasi-stationary equations described above, where the left electron hole initially has the speed $M_{\mathrm{e}}=0.7$ and is loaded with electromagnetic waves with $W_{\max }=1.5$, while the right electron hole has the speed $M_{\mathrm{e}}=-0.3$, and is loaded with electromagnetic waves with $W_{\max }=2.5$. Further, we used $k_{0}=v_{\mathrm{g}}=0$ in the initial condition for $A$ and in the solution of Eq. (172). In Fig. 43 we show the phase-space distribution of the electrons and the electromagnetic field amplitude at different times. We see that the relativistic electron holes loaded with trapped electromagnetic waves collide, merge and then split into two electron holes, while there are a few strongly peaked electromagnetic wave envelopes at $x \approx 70$ remaining after the splitting of the relativistic electron hole, and where a population of electrons has been accelerated to large energies. The time development of the electromagnetic wave amplitudes, relativistic electron hole potential, the squared local plasma frequency and the electron number density is shown in Fig. 44. We observe collision and splitting of the relativistic electron holes and the creation of localized electromagnetic solitary waves at $z \approx 70$; clearly visible in the left two panels at $t>150$. The electromagnetic solitary waves are created by the combined action of the relativistic electron mass increase and relativistic ponderomotive force of localized electromagnetic waves, which have been further intensified due to nonlinear interactions where the collapsing relativistic electron hole has deposited its energy into the plasma. 

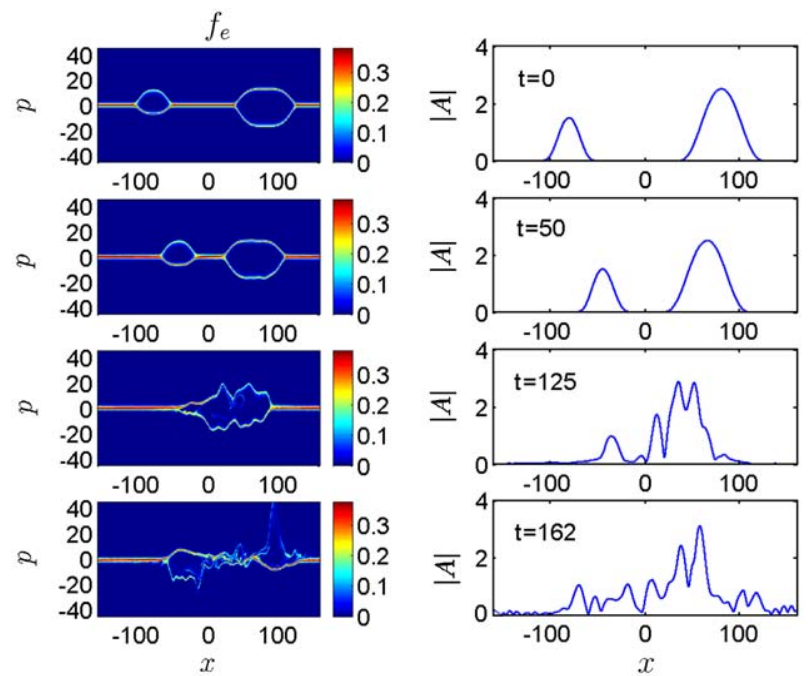

Fig. 43. Phase-space plots of the electron distribution function $f_{\mathrm{e}}$ (left panels) and the amplitude of the vector potential $A$ (right panels) for $t=0$, 50, 125 and 162 (after Ref. [155]).
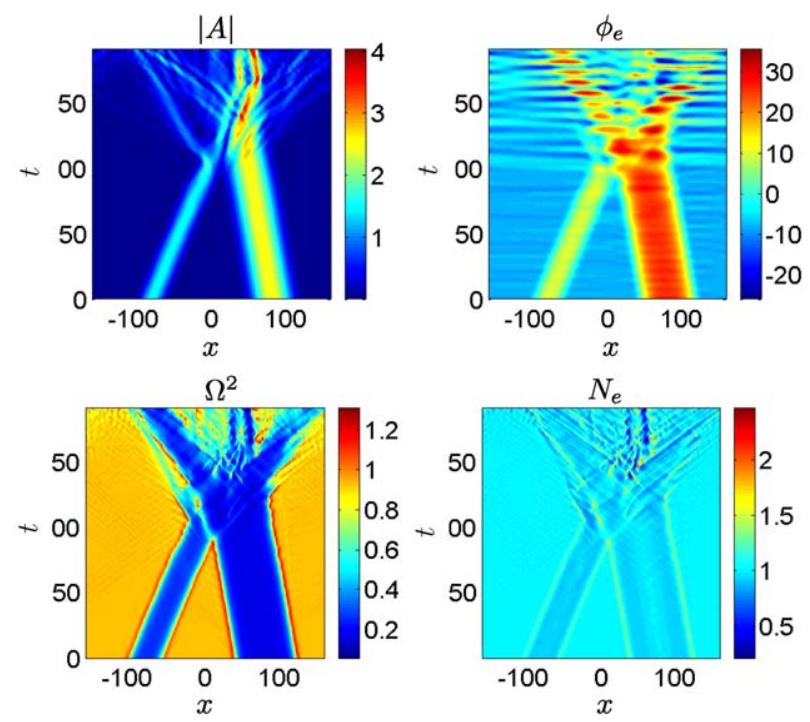

Fig. 44. The amplitude of the vector potential (upper left panel), potential (upper right panel), squared local plasma frequency (lower left panel) and electron density (lower right panel) for two colliding relativistic electron holes (after Ref. [155]).

\section{Summary and conclusions}

In this paper, we have reviewed the formation and dynamics of coherent nonlinear structures involving phasespace vortices in electron-ion and electron-positron (or pair-ion) plasmas that is free of either an external magnetic field or embedded in it. We have discussed the underlying physics of the electron and ion holes as well as double layers and presented appropriate models that describe their stationary features and existence regimes. We note that a new and largely unexplained type of double layers with tripolar electric fields have been observed in the Earth's ionosphere/magnetosphere and in solar wind by numerous spacecrafts. In studying the dynamics of ion and electron holes, analytic solutions of quasi-stationary electron and ion holes have been employed as initial conditions in computer simulations of the Vlasov-Poisson system, in order to understand the complex nonlinear dynamics of those nonlinear 
structures in an electron-ion plasma. We observe that electron holes in a plasma with mobile ions show an interesting dynamics when the speed of the electron hole is small enough. A standing electron hole accelerates locally the ions due to the positive electron hole potential. The self-created ion density cavity, on the other hand, accelerates the electron hole, which leaves the ion cavity and propagates with a constant speed. The propagating electron hole can be trapped at ion density maxima, where it again accelerates the ions locally and a new ion density cavity is formed, etc. On the other hand, colliding ion holes may create non-Maxwellian electron distributions due to the acceleration of electrons in the negative ion hole potential. This can create streams of accelerated electrons before and during the ion hole collisions, and a flat-topped electron distribution between the ion holes, after collisions. The accelerated streams of electrons can excite high-frequency Langmuir waves due to a streaming instability. Thus, both ion and electron holes show an interesting and complex dynamics during their interaction with the background plasma, with a variety of couplings between fast and slow timescales. Knowledge of dynamically evolving electron and ion holes and their interaction with the background plasma is required for understanding the properties of localized electric pulses in the Earth's auroral zone and in the magnetosphere.

We have also presented theoretical and simulation studies of the formation and dynamics of holes in a non-relativistic pair plasma (electron-positron or positive and negative ions), which is found in astrophysical environments and in laboratory experiments. The existence of large amplitude ion holes with a maximum speed of the same order as the ion thermal speed has been established. Also presented is a theory for relativistic electron holes (REHs). Due to the relativistic electron mass increase, the size of the electron hole as well as the amplitude of the electron hole potential increase dramatically with increasing relativistic electron temperature. Simulation studies of interactions between REHs show that they interact in a complex fashion, and may merge when they collide, resulting in accelerated particles and a release of the electromagnetic radiation.

The present review gives a flavor of electron trapping in a magnetized plasma. Here, the external magnetic field affects the electron distribution function and is responsible for asymmetric electron holes whose scale sizes along and across the magnetic field direction are different. Such coherent structures have indeed been observed by numerous spacecrafts in the Earth's auroral zone and in the magnetosphere. Our theoretical models support the space observations. It should be mentioned that a recent laboratory experiment [33] has conclusively demonstrated the formation of electrostatic DLs and electron holes in a magnetized low-pressure mercury plasma column. The magnetic field locally increases the electron current density and makes the DL form at the center of the magnetic constriction where it remained at rest. Here, one also observes a pure electron hole, which is a symmetric triple-layer having a bell-shape potential profile. However, no theoretical explanation currently exists for this experimental observation.

The presence of large amplitude Langmuir and electromagnetic waves provides interesting nonlinear couplings with electron and ions holes in plasmas. Nonlinear couplings come about due to the ponderomotive force for the Langmuir wave case, and due to the combined action of relativistic mass increase and the relativistic ponderomotive force for the intense electromagnetic wave case. In the high-frequency Langmuir wave case, one observes the high-frequency wave trapping by ion holes, while intense electromagnetic waves are trapped by the REHs that we discussed above. Plasmas with intense electrostatic fields, which are driven by localized envelops of Langmuir and intense electromagnetic waves, are suited for charged particle acceleration involving collective interactions. Specifically, ultra-high gradients of electrostatic fields can cause acceleration of electrons to extremely energies in relativistically hot plasmas, such as those in inertial confinement fusion as well as in compact astrophysical objects.

In conclusion, the results contained in this review article should help to understand the salient features of coherent nonlinear structures in non-equilibrium plasmas which contain particle distributions that are far away from the Maxwellian. Such non-isothermal particle distributions and associated electrostatic structures are frequently found in space and laboratory experiments. We hope that the present review will be useful to understand the essentials of kinetic structures which cannot be treated by means of existing fluid theories.

\section{Outlook and future directions}

As we have seen, coherent nonlinear structures involving non-isothermal particle distributions are unique in that they have to be modeled with the Vlasov and Maxwell equations. Complexities occur when the plasma contains ambient magnetic fields and plasma density gradients, a scenario quite common in space and laboratory environments. Observations from on-board instruments of numerous spacecrafts (e.g. Freja [164], FAST [47,48], POLAR [42], etc.) 
have revealed 3D structures which are not fully understood. Self-consistent calculations of non-isothermal distribution functions in non-uniformed magnetized plasmas with large amplitude localized electrostatic fields are rather intricate and challenging. However, physical insight of a particular localized structure can be gained by modelling distribution functions that are consistent with observations. For example, it would be worthwhile to formulate a theory for tripolar electrostatic fields which are anisotropic with respect to the magnetic field direction. This will require the knowledge of non-isothermal electron and ion distribution functions and associated density distributions and localized electric fields in the presence of a magnetic field. The dynamics of 3D electron and ion holes in magnetized space plasmas has been numerically studied in the small-amplitude limit by employing Schamel's distribution functions for the trapped and free particles [165,166]. Furthermore, attempts have also been made [167,168] to construct 3D BGK solitary waves on the basis of steady-state Vlasov and Poisson equations in magnetized plasmas. Here, there is a new direction in obtaining possible solutions in the form of spatially localized axially symmetric potential hump structures associated with electron holes in the cylindrical coordinates. One should also carry out simulations of the multi-dimensional Vlasov-Poisson system of equations with some initial nonthermal particle distribution functions and associated 3D BGK solitary waves, in order to study the dynamics and stability of the observed structures in magnetized plasmas.

The electron and ion holes have the tendency to trap Langmuir and electromagnetic waves under certain conditions. In a magnetized plasma, upper-hybrid and electromagnetic electron-cyclotron waves may be trapped in two- and 3D electron and ion holes. Furthermore, relativistic electron holes in a magnetized plasma could be expected to have a complex profile. It is anticipated that magnetic fields may produce 3D stable localized structures, which can trap intense electromagnetic waves that are affected by the magnetic field itself. We note that recent observations from laser-plasma interaction experiments [169] reveal a signature of gigagauss magnetic fields. In such a situation, there may also exist possibilities of pair creation and ultra-intense electric fields. The latter can serve the purpose of accelerating electrons to extremely high energies. We are hoping that plasma-based charged particle accelerators involving kinetic electrostatic structures would be able to provide clues to the origin of gamma-ray bursts and ultra-high energy cosmic rays. In fact, future analytical and simulation investigations shall concentrate on demonstrating the efficiency of accelerating charged particles (electrons, positrons and protons) by intense localized coherent structures and fields that involve non-isothermal particle distribution functions in non-uniform magnetized plasmas. Computer simulations [93] and observations [170] also indicate that both the magnetic field and relativistic effects play a major role in the dynamics of BGK modes which are decisive for the subsequent surfing acceleration [171-173] of charged particles to highly relativistic energies.

\section{Acknowledgements}

The authors acknowledge the valuable contributions of Hans Schamel who pioneered the field of kinetic structures in plasmas, as well as of Dusan Jovanović who was deeply involved with the development of theories for phase-space holes in magnetized plasmas. We thank both of them for many useful discussions. We also thank an anonymous referee for useful suggestions that improved the presentation of our paper.

This work was partially supported by the Deutsche Forschungsgemeinschaft (Bonn) through the Sonderforschungsbereich 591, as well as by the European Commission (Brussels) through Contract No. HPRN-CT-2001-00314 for carrying out the tasks of the research training network entitled "Turbulent Boundary Layers in Geospace Plasmas". The Swedish National Supercomputer Centre (NSC) provided the computer resources to carry out this work.

\section{References}

[1] R.Z. Sagdeev, in: M.A. Leontovich (Ed.), Reviews of Plasma Physics, vol. 4, Consultants Bureau, New York, 1966, pp. 23.

[2] R.Z. Sagdeev, The 1976 Oppenheimer lectures: critical problems in plasma astrophysics. II. Singular layers and reconnection, Rev. Mod. Phys. 51 (1979) 11-20.

[3] I.B. Bernstein, J.M. Greene, M.D. Kruskal, Exact nonlinear plasma oscillations, Phys. Rev. 108 (1957) 546-550.

[4] D. Andersson, L. Helczynski-Wolf, M. Lisak, V. Semenov, Features of modulational instability of partially coherent light: importance of the incoherence spectrum, Phys. Rev. E 69 (2004) 025601/1-4.

[5] R.A. Dory, Mass-conjugate instabilities, J. Nucl. Energy, Part C Plasma Phys. 6 (1964) 511-512.

[6] K.V. Roberts, H.L. Berk, Nonlinear evolution of a two-stream instability, Phys. Rev. Lett. 19 (1967) 297-300.

[7] P.H. Sakanaka, Formation and interaction of ion-acoustic solitary waves in a collisionless warm plasma, Phys. Fluids 15 (1972) $304-310$. 
[8] D.L. Newman, M.V. Goldman, M. Spector, F. Perez, Dynamics and instability of electron phase-space tubes, Phys. Rev. Lett. 86 (2001) 1239-1242.

[9] L.K.S. Daldorff, P. Guio, S. Børve, H.L. Pécseli, J. Trulsen, Ion phase space vortices in 3 spatial dimensions, Europhys. Lett. 54 (2001) $161-167$.

[10] V.L. Krasovsky, H. Matsumoto, Y. Omura, Interaction dynamics of electrostatic solitary waves, Nonlinear Proc. Geophys. 6 (1999) $205-209$.

[11] G. Vetoulis, M. Oppenheim, Electrostatic mode excitation in electron holes due to wave bounce resonances, Phys. Rev. Lett. 86 (2001) $1235-1238$.

[12] P. Guio, S. Børve, L.K.S. Daldorff, J.P. Lynov, P. Michelsen, H.L. Pécseli, J.J. Rasmussen, K. Saeki, J. Trulsen, Phase space vortices in collisionless plasmas, Nonlinear Proc. Geophys. 10 (2003) 75-86.

[13] P. Guio, H.L. Pécseli, Phase space structures generated by an absorbing obstacle in a streaming plasma, Geophys. Res. Lett. 31 (2004) L03806/1-4, doi: 10.1029/2003GL018461.

[14] K. Saeki, H. Genma, Electron-hole disruption due to ion motion and formation of coupled hole and ion-acoustic soliton in a plasma, Phys. Rev. Lett. 80 (1998) 1224-1227.

[15] M.V. Medvedev, P.H. Dimond, M.N. Rosenbluth, V.I. Shevchenko, Asymptotic theory of nonlinear Landau damping and particle trapping in waves of finite amplitude, Phys. Rev. Lett. 81 (1998) 5824-5826.

[16] C. Lancellotti, J.J. Dorning, Nonlinear Landau damping in a collisionless plasma, Phys. Rev. Lett. 80 (1998) 5236.

[17] G. Manfredi, Long-time behavior of nonlinear Landau damping, Phys. Rev. Lett. 79 (1997) 2815-2818.

[18] T.H. Dupree, Theory of phase-space density holes, Phys. Fluids 25 (1982) 277-289.

[19] J.P. Holloway, J.J. Dorning, Undamped plasma waves, Phys. Rev. A 44 (1991) 3856-3868.

[20] H. Schamel, Hole equilibria in Vlasov-Poisson systems: a challenge to wave theories of ideal plasmas, Phys. Plasmas 7 (2000) $4831-4844$.

[21] H. Abbasi, N.L. Tsintsadze, D.D. Tskhakaya, Influence of particle trapping on the propagation of ion cyclotron waves, Phys. Plasmas 6 (1999) 2373-2379.

[22] H. Schamel, Stationary solutions of the electrostatic Vlasov equation, Plasma Phys. 13 (1971) 491-505.

[23] H. Schamel, Stationary solitary, snoidal and sinusoidal ion acoustic waves, Plasma Phys. 14 (1972) 905-924.

[24] H. Schamel, Theory of electron holes, Phys. Scripta 20 (1979) 336-342.

[25] H. Schamel, Electron holes, ion holes and double layers, Phys. Rep. 140 (1986) 161-191.

[26] S. Bujarbarua, H. Schamel, Theory of electron and ion holes, J. Plasma Phys. 25 (1981) 515-529.

[27] H. Schamel, Stability of electron vortex structures in phase space, Phys. Rev. Lett. 48 (1982) 481-483.

[28] H. Schamel, R. Fedele, Kinetic theory of solitary waves on coasting beams in synchrotrons, Phys. Plasmas 7 (2000) 3421-3430.

[29] L. Stenflo, N.L. Tsintsadze, T.D. Buadze, Solitary acoustic waves in weakly ionized gases, Phys. Lett. A 135 (1989) 37-38.

[30] R. Fedele, Envelope Solitons versus Solitons, Phys. Scripta 65 (2002) 502-508.

[31] R. Fedele, H. Schamel, Solitary waves in the Madelung's fluid: connection between the nonlinear Schrödinger equation and the Kortewegde Vries equation, Eur. Phys. J. B 27 (2002) 313-320.

[32] K. Saeki, P. Michelsen, H.L. Pécseli, J.J. Rasmussen, Formation and coalescence of electron solitary holes, Phys. Rev. Lett. 42 (1979) 501-504.

[33] G. Petraconi, H.S. Maciel, Formation of electrostatic double-layers and electron-holes in a low pressure mercury plasma column, J. Phys. D: Appl. Phys. 36 (2003) 2798-2805.

[34] H.L. Pécseli, J. Trulsen, R.J. Armstrong, Experimental observation of ion phase-space vortices, Phys. Lett. 81A (1981) 386-390.

[35] H.L. Pécseli, J. Trulsen, R.J. Armstrong, Formation of ion phase-space vortices, Phys. Scripta 29 (1984) 241-253.

[36] H.L. Pécseli, Ion phase-space vortices and their relation to small amplitude double-layers, Laser Part. Beams 5 (1987) $211-217$.

[37] Y. Nakamura, H. Bailung, P.K. Shukla, Observation of ion-acoustic shocks in a dusty plasma, Phys. Rev. Lett. 83 (1999) $1602-1605$.

[38] C. Franck, T. Klinger, A. Piel, H. Schamel, Dynamics of periodic ion holes in a forced beam-plasma experiment, Phys. Plasmas 8 (2001) 4271-4274.

[39] A.A. Kabantsev, J.H. Yu, R.B. Lynch, F.F. Driscoll, Trapped particles and asymmetry-induced transport, Phys. Plasmas 10 (2003) $1628-1635$.

[40] M. Temerin, K. Cherny, W. Lotko, F.S. Mozer, Observations of double layers and solitary waves in the auroral plasma, Phys. Rev. Lett. 48 (1982) 1175-1179.

[41] R. Boström, G. Gustafsson, B. Holback, G. Holmgren, H. Koskinen, P. Kintner, Characteristics of solitary waves and weak double layers in the magnetospheric plasma, Phys. Rev. Lett. 61 (1988) 82-85.

[42] J. Dombeck, C. Cattell, J. Crumley, W.K. Peterson, H.L. Collin, C. Kletzing, Observed trends in auroral zone ion mode solitary wave structure characteristics using data from Polar, J. Geophys. Res. Space Phys. 106 (2001) 19013-19022.

[43] C. Cattell, J. Crumley, J. Dombeck, J. Wygant, F.S. Mozer, Polar observations of solitary waves at the Earth's magnetopause, Geophys. Res. Lett. 29 (2002) 1065.

[44] J.P. McFadden, C.W. Carlson, R.E. Ergun, D.M. Klumpar, E. Moebius, Ion and electron characteristics in auroral density cavities associated with ion beams: no evidence for cold ionospheric plasma, J. Geophys. Res. Space Phys. 104 (1999) 14671-14682.

[45] J.P. McFadden, C.W. Carlson, R.E. Ergun, F.S. Mozer, L. Muschietti, I. Roth, E. Moebius, FAST observations of ion solitary waves, J. Geophys. Res. 108 (2003) 8018, COA 19-1 - 19-17, doi: 10.1029/2002JA009485.

[46] H. Matsumoto, H. Kojima, T. Miyatake, Y. Omura, M. Okada, I. Nagano, M. Tsutsui, Electrostatic solitary waves (ESW) in the magnetotail: BEN wave forms observed by Geotail, Geophys. Res. Lett. 21 (1994) 2915-2918;

H. Matsumoto, H. Kojima, T. Miyatake, Y. Omura, M. Okada, I. Nagano, M. Tsutsui, Relation between electrostatic solitary waves and hot plasma flow in the plasma sheet boundary layer: Geotail observations, Geophys. Res. Lett. 21 (1994) 2919-2922.

[47] R.E. Ergun, C.W. Carlson, J.P. McFadden, F.S. Mozer, L. Muschietti, I. Roth, R.J. Strangeway, Debye-scale plasma structures associated with magnetic-field-aligned electric fields, Phys. Rev. Lett. 81 (1998) 826-829. 
[48] R.E. Ergun, C.W. Carlson, J.P. McFadden, F.S. Mozer, G.T. Delory, W. Peria, C.C. Chaston, M. Temerin, I. Roth, L. Muschietti, R. Elphik, R. Strangeway, R. Pfaff, C. Cattell, D. Klumpar, E. Shelly, W. Peterson, E. Moebius, L. Kistler, FAST satellite observations of large-amplitude solitary structures, Geophys. Res. Lett. 25 (1998) 2041-2044.

[49] R.E. Ergun, Y.-J. Su, L. Andersson, C.W. Carlson, J.P. McFadden, F.S. Mozer, D.L. Newman, M.V. Goldman, R.J. Strangeway, Direct observation of localized parallel electric fields in a space plasma, Phys. Rev. Lett. 87 (2001) 45003/1-4.

[50] S.D. Bale, P.J. Kellogg, D.E. Larson, R.P. Lin, K. Goetz, R.P. Lepping, Bipolar electrostatic structures in the shock transition region, Evidence of electron phase space holes, Geophys. Res. Lett. 25 (1998) 2929-2932.

[51] S.D. Bale, A. Hull, D.E. Larson, R.P. Lin, L. Muschietti, P.J. Kellogg, K. Goetz, S.J. Monson, Electrostatic turbulence and Debye-scale structures associated with electron thermalization at collisionless shocks, Astrophys. J. 575 (2002) L25-L28.

[52] M. Hoshino, Coupling across many scales, Science 299 (2003) 834-835.

[53] F.S. Mozer, R. Ergun, M. Temerin, C. Cattell, J. Dombeck, J. Wygant, New features of time domain electric field structures in the auroral acceleration region, Phys. Rev. Lett. 79 (1997) 1281-1284.

[54] J.S. Pickett, J.D. Menietti, D.A. Gurnett, B. Tsurutani, P.M. Kintner, E. Klatt, A. Balogh, Solitary potential structures observed in the magnetosheath by the cluster spacecraft, Nonlinear Proc. Geophys. 9 (2002) 1-9 [Also in Nonlin. Proc. Geophys. 10 (2003) 3-11].

[55] J.S. Pickett, S.W. Kahler, L.-J. Chen, R.L. Huff, O. Santolik, Y. Khotyaintsev, P.M.E. Décréau, D. Winningham, R. Frahm, M.L. Goldstein, G.S. Lakhina, B.T. Tsurutani, B. Lavraud, D.A. Gurnett, M. André, A. Fazakerley, A. Balogh, H. Rème, Solitary waves observed in the auroral zone: the cluster multi-spacecraft perspective, Nonlinear Proc. Geophys. 11 (2004) 183-196.

[56] J.F. Drake, M. Swisdak, C. Cattell, M.A. Shay, B.N. Rogers, A. Zeiler, Formation of electron holes and particle energization during magnetic reconnection, Science 299 (2003) 873-877.

[57] C. Cattell, J. Domebeck, J. Wygant, J. F. Drake, M. Swisdak, M. L. Goldstein, W. Keith, A. Fazakerley, M. André, E. Lucek, A. Balogh, Cluster observations of electron holes in association with magnetotail reconnection and comparison to simulations, J. Geophys. Res. 110 (2005) A01211/1-16, doi: 10.1029/2004JA010519.

[58] J.R. Franz, P.M. Kintner, andJ.S. Pickett, POLAR observations of coherent electric field structures, Geophys. Res. Lett. 25 (1998) 1277-1280.

[59] A. Hasegawa, Stimulated modulational instabilities of plasma waves, Phys. Rev. A 1 (1970) 1746-1750.

[60] V.I. Karpman, High frequency electromagnetic field in plasma with negative dielectric constant, Plasma Phys. 13 (1971) $477-490$.

[61] V.I. Karpman, On the dynamics of sonic-Langmuir solitons, Phys. Scripta 11 (1975) 263-265.

[62] V.I. Karpman, Nonlinear Waves in Dispersive Media, Pergamon, Oxford, 1975.

[63] V.E. Zakharov, Collapse of Langmuir waves, Zh. Eksp. Teor. Fiz. 62 (1972) 1745-1751 [Sov. Phys. JETP 35 (1972) 908-914].

[64] L.I. Rudakov, Deceleration of electron beams in a plasma with a high level of Langmuir turbulence, Dokl. Akad. Nauk SSSR 207 (1972) 821 -823 [Sov. Phys. Dokl. 17 (1973) 1166].

[65] R.K. Varma, N.N. Rao, Theory of sonic-Langmuir solitons, Phys. Lett. A 79 (1980) 311-314.

[66] N.N. Rao, R.K. Varma, A theory for Langmuir solitons, J. Plasma Phys. 27 (1982) 95-120.

[67] H. Schamel, M.Y. Yu, P.K. Shukla, Finite amplitude envelope solitons, Phys. Fluids 20 (1977) 1286-1288.

[68] H. Schamel, P.K. Shukla, Envelope solitons in the presence of nonisothermal electrons, Phys. Rev. Lett. 36 (1976) $968-972$.

[69] H. Schamel, V. Maslov, Langmuir contraction caused by electron holes, Phys. Scripta T 82 (1999) 122-124.

[70] V.V. Yan'kov, Two types of Langmuir solitons, Pis'ma Zh. Eksp. Teor. Fiz. 29 (1979) 179-180 [JETP Lett. 29 (1979) 160-161].

[71] Yu.V. Mokhov, K.V. Chukbar, Langmuir solitons of the Bernstein-Greene-Kruskal type, Fiz. Plazmy 10 (1984) 206-208 [Sov. J. Plasma Phys. 10 (1984) 122-123].

[72] F. Califano, M. Lontano, Vlasov simulations of strongly nonlinear electrostatic oscillations in a one-dimensional electron-ion plasma, Phys. Rev. E 58 (1998) 6503-6511.

[73] J.G. Wang, D.L. Newman, M.V. Goldman, Vlasov simulations of electron heating by Langmuir turbulence near the critical altitude in the radiation-modified ionosphere, J. Atm. Sol.-Terr. Phys. 59 (1997) 2461-2474.

[74] V.D. Shapiro, V.I. Shevchenko, in: A.A. Galeev, R.N. Sudan (Eds.), Handbook of Plasma Physics, vol. II, North-Holland, Amsterdam, 1984, p. 8.

[75] M.V. Goldman, Strong turbulence of plasma waves, Rev. Mod. Phys. 56 (1984) 709-735.

[76] H.C. Wong, R. Stenzel, A.Y. Wong, Development of "cavitons" and trapping of rf field, Phys. Rev. Lett. 33 (1974) 886-889.

[77] H. Ikezi, R.P.H. Chang, R.A. Stern, Nonlinear evolution of the electron-beam-plasma instability, Phys. Rev. Lett. 36 (1976) $1047-1051$.

[78] T. Intrator, C. Chan, N. Hershkowitz, D. Diebold, Nonlinear self-contraction of electron waves, Phys. Rev. Lett. 53 (1984) $1233-1235$.

[79] A.Y. Wong, B.H. Quon, Spatial collapse of beam-driven plasma waves, Phys. Rev. Lett. 34 (1975) 1499-1502.

[80] P.K. Shukla, N.N. Rao, M.Y. Yu, N.L. Tsintsadze, Relativistic nonlinear effects in plasmas, Phys. Rep. 138 (1986) 1-149.

[81] D.S. Montgomery, R.J. Focia, H.A. Rose, D.A. Russell, J.A. Cobble, J.C. Fernández, R.P. Johnson, Observation of stimulated electronacoustic-wave scattering, Phys. Rev. Lett. 87 (2001) 155001/1-4.

[82] R. Bingham, Accelerator physics: in the wake of success, Nature (London) 424 (2003) 258-259.

[83] R. Bingham, J.T. Mendonça, P.K. Shukla, Plasma based charged-particle accelerators, Plasma Phys. Control Fusion 46 (2004) R1-R23.

[84] J.T. Mendonça, Theory of Photon Acceleration, Institute of Physics, Bristol, 2001.

[85] T. Piran, Gamma-ray bursts and the fireball model, Phys. Rep. 314 (1999) 575-667.

[86] V.A. Kozlov, A.G. Litvak, E.V. Suvorov, Envelope solitons of relativistic strong electromagnetic waves, Sov. Phys. JETP 49 (1979) $75-80$.

[87] P.K. Kaw, A. Sen, T. Katsouleas, Nonlinear 1D laser pulse solitons in a plasma, Phys. Rev. Lett. 68 (1992) 3172-3175.

[88] T.Zh. Esirkepov, F.F. Kamenets, S.V. Bulanov, N.M. Naumova, Low-frequency relativistic electromagnetic solitons in collisionless plasmas, JETP Lett. 68 (1998) 36-41.

[89] S.V. Bulanov, T.Zh. Esirkepov, N.M. Naumova, F. Pegoraro, V.A. Vshivkov, Solitonlike electromagnetic waves behind a superintense laser pulse in a plasma, Phys. Rev. Lett. 82 (1999) 3440-3443. 
[90] D. Farina, S.V. Bulanov, Relativistic electromagnetic solitons in the electron-ion plasma, Phys. Rev. Lett. 86 (2001) 5289-5292.

[91] N.M. Naumova, S.V. Bulanov, T.Zh. Esirkepov, D. Farina, K. Nishihara, F. Pegoraro, H. Ruhl, A.S. Sakharov, Formation of electromagnetic postsolitons in plasmas, Phys. Rev. Lett. 87 (2001) 185004/1-4.

[92] M. Borghesi, S. Bulanov, D.H. Campbell, R.J. Clarke, T.Zh. Esirkepov, M. Galimberti, L.A. Gizzi, A.J. MacKinnon, N.M. Naumova, F. Pegoraro, H. Ruhl, A. Schiavi, O. Willi, Macroscopic evidence of soliton formation in multiterawatt laser-plasma interaction, Phys. Rev. Lett. 88 (2002) 135002/1-4.

[93] M.E. Dieckmann, B. Eliasson, P.K. Shukla, Streaming instabilities driven by mildly relativistic proton beams in plasmas, Phys. Plasmas 11 (2004) 1394-1401.

[94] W.L. Kruer, J.M. Dawson, R.N. Sudan, Trapped-particle instability, Phys. Rev. Lett. 23 (1969) 838-841.

[95] M.V. Goldman, Theory of stability of large periodic plasma waves, Phys. Fluids 13 (1970) 1281-1289.

[96] M.E. Dieckmann, B. Eliasson, A. Stathopoulos, A. Ynnerman, Connecting shock velocities to electron-injection mechanisms, Phys. Rev. Lett. 92 (2004) 065006/1-4.

[97] H. Schamel, S. Bujarbarua, Solitary plasma hole via ion-vortex distribution, Phys. Fluids 23 (1980) 2498-2499.

[98] L.P. Block, in: B. Hultqvist, L. Stenflo (Eds.), Physics of the Hot Plasma in the Magnetosphere, Plenum, New York, 1975, pp. 229-249.

[99] J.S. Levine, F.W. Crawford, A fluid description of plasma double-layers, J. Plasma Phys. 23 (1980) 223-247.

[100] A. Hasegawa, T. Sato, Existence of negative potential solitary-wave structure and formation of a double layer, Phys. Fluids 25 (1982) 632-635.

[101] K.Y. Kim, Weak monotonic double layers, Phys. Lett. 97 A (1983) 45-51.

[102] D.L. Newman, M.V. Goldman, R.E. Ergun, A. Mangeney, Formation of double layers and electron holes in a current-driven space plasma, Phys. Rev. Lett. 87 (2001) 255001/1-4.

[103] H. Schamel, Weak double layers: existence, stability and evidence, Z. Naturforsch. 38 (1983) 1170-1183.

[104] A. Mangeney, C. Salem, C. Lacombe, J.-L. Bougeret, C. Perche, R. Manning, P.J. Kellogg, K. Goertz, S.L. Monson, J.-M. Bosqued, WIND observations of coherent electrostatic waves in the solar wind, Ann. Geophys. 17 (1999) 307-320.

[105] C. Lacombe, J.L. Bougeret, C. Perche, R. Manning, P. Kellogg, K. Goetz, S. Monson, J.M. Bosqued, Evidence for the interplanetary electric potential? WIND observations of electrostatic fluctuations, Ann. Geophys. 20 (2002) 609-618.

[106] H. Schamel, Kinetic theory of phase space vortices and double layers, Phys. Scripta T2 (1982) 228-237.

[107] D. Jovanović, P.K. Shukla, G. Morfill, A nonlinear model for coherent tripolar electric field structures in the Earth's auroral zone and solar wind, J. Plasma Phys. 71 (2005) 203-211.

[108] B. Eliasson, P.K. Shukla, Dynamics of electron holes in an electron-oxygen-ion plasma, Phys. Rev. Lett. 91 (2004) 045001/1-4.

[109] B. Eliasson, The parallel implementation of the one-dimensional Fourier transformed Vlasov-Poisson system, Comput. Phys. Commun. 170 (2005) 205-230.

[110] B. Eliasson, P.K. Shukla, Production of nonisothermal electrons and Langmuir waves because of colliding ion holes and trapping of plasmons in an ion hole, Phys. Rev. Lett. 92 (2004) 95006/1-4.

[111] P.K. Shukla, B. Eliasson, Trapping of plasmons in ion holes, JETP Lett. 77 (2003) 647-652 [Zh. Eksp. Teor. Fiz. 77 (2003) 778-783].

[112] M.J. Rees, in: G.B. Gibbons, S.W. Hawking, S. Sikias (Eds.), The Very Early Universe, Cambridge University Press, Cambridge, 1983.

[113] Positron-Electron Pairs in Astrophysics, M. L. Burns et al. (Eds.), AIP, New York, 1983.

[114] F.C. Michel, Theory of pulsar magnetospheres, Rev. Mod. Phys. 54 (1986) 1-66.

[115] T. Piran, The physics of gamma-ray bursts, Rev. Mod. Phys. 76 (2004) 1143-1210.

[116] N.C. Woolsey, C. Courtois, R.O. Dendy, Laboratory plasma astrophysics simulation experiments using lasers, Plasma Phys. Control Fusion 46 (2004) B397-B405.

[117] V.I. Berezhiani, D.D. Tskhakaya, P.K. Shukla, Pair production in a strong wake field driven by an intense short laser pulse, Phys. Rev. A 46 (1992) 6608-6612.

[118] P. Helander, D.J. Ward, Positron creation and annihilation in tokamak plasmas with runaway electrons, Phys. Rev. Lett. 90 (2003) 135004/1-4.

[119] R. Alkofer, M.B. Hecht, C.D. Roberts, S.M. Schmidt, D.V. Vinnik, Pair creation and an X-ray free electron laser, Phys. Rev. Lett. 87 (2001) 193902/1-4.

[120] W. Oohara, D. Date, R. Hatakeyama, Electrostatic waves in a paired fullerene-ion plasma, Phys. Rev. Lett. 95 (2005) 175003/1-4.

[121] H. Schamel, A. Luque, Kinetic theory of periodic hole and double layer equilibria in pair plasmas, New J. Phys. 7 (2005) 69/1-9.

[122] B. Eliasson, P.K. Shukla, Solitary phase-space holes in pair plasmas, Phys. Rev. E 71 (2005) 046402/1-5.

[123] W. Oohara, R. Hatakeyama, Pair-ion plasma generation using fullerenes, Phys. Rev. Lett. 91 (2004) 205005/1-4.

[124] W. Oohara, S. Ishiguro, R. Hatakeyama, N. Sato, Dynamic evolution of plasma structures due to local production of massive negative ions, J. Phys. Soc. Jpn. 71 (2002) 373-376.

[125] W. Oohara, R. Hatakeyama, S. Ishiguro, Plasma state transition originating from local production of massive negative ions, Phys. Rev. E 68 (2003) 066407/1-8.

[126] N. Meyer-Vernet, Flip-flop of electric potential of dust grains in space, Astron. Astrophys. 105 (1982) 98-106.

[127] M. Horányi, C.K. Goertz, Coagulation of dust particles in a plasma, Astrophys. J. 361 (1990) 155-161.

[128] B. Smiley, S. Robertson, M. Horányi, T. Blix, M. Rapp, R. Latteck, J. Gumbel, Measurement of positively and negatively charged particles inside PMSE during MIDAS SOLSTICE 2001, J. Geophys. Res. Atm. 108 (2003) 8444.

[129] B. Eliasson, P.K. Shukla, Theory of relativistic electron holes in hot plasmas, Phys. Lett. A 338 (2005) 237-242.

[130] S.R. de Groot, W.A. van Leeuwen, C.G. Weert, Relativistic Kinetic Theory, Principles and Applications, North-Holland, Amsterdam, 1980.

[131] I.S. Gradshteyn, I.M. Ryzhik, Table of Integrals, Series, and Products, fourth ed., Academic, New York, 1965.

[132] S.K. Lele, Compact finite difference schemes with spectral-like resolution, J. Comput. Phys. 103 (1992) 16-42.

[133] D. Jovanović, P.K. Shukla, L. Stenflo, F. Pegoraro, Nonlinear model for electron phase-space holes in magnetized space plasmas, J. Geophys. Res. Space Phys. 107 (2002) 1110. 
[134] Y. Omura, H. Matsumoto, T. Miyake, H. Kojima, Electron beam instabilities as generation mechanism of electrostatic solitary waves in the magnetotail, J. Geophys. Res. Space Phys. 101 (1996) 2685-2698.

[137] N. Singh, S.M. Loo, B. Earl Wells, C. Deverapalli, Three-dimensional structure of electron holes driven by an electron beam, Geophys. Res. Lett. 27 (2000) 2469-2472.

[138] D. Jovanović, F. Pegoraro, Kinetic vortex chain solution in the drift-wave plasma regime, Phys. Rev. Lett. 84 (2000) 95-98.

[139] D. Jovanović, P.K. Shukla, Nonlinear model for coherent electric field structures in the magnetosphere, Phys. Rev. Lett. 84 (2000) $4373-4376$.

[140] D.A. D'Ippolito, R.C. Davidson, A hybrid kinetic model for high- $\beta$ plasmas, Phys. Fluids 18 (1975) 1507-1523.

[143] H. Schamel, V.I. Maslov, Adiabatic growth of electron holes in current-carrying plasmas, Phys. Scripta T50 (1994) $42-46$.

[144] H. Schamel, Theory of solitary holes in coasting beams, Phys. Rev. Lett. 79 (1997) 2811-2814.

[145] B.N. Kuvshinov, F. Pegoraro, J. Rem, T.J. Schep, Drift-Alfvén vortices with finite ion gyroradius and electron inertia effects, Phys. Plasmas 6 (1999) 713-728.

[146] D. Jovanović, P.K. Shukla, Formation of nonlinear magnetic islands via trapped electrons in the lower hybrid-range, Phys. Plasmas 11 (2004) 4946-4953.

[147] D. Jovanović, P.K. Shukla, Magnetic reconnection mediated by lower-hybrid phase-space vortices, Phys. Rev. Lett. 93 (2004) 015002/1-4.

[148] D. Jovanović, P.K. Shukla, Linear theory for fast collisionless magnetic reconnection in the lower-hybrid frequency range, Phys. Plasmas 12 (2005) 052114/1-10.

[149] X.H. Deng, H. Matsumoto, Rapid magnetic reconnection in the Earth's magnetosphere mediated by whistler waves, Nature (London) 410 (2001) 557-559.

[150] F.S. Mozer, S.D. Bale, T.D. Phan, Evidence of diffusion regions at a subsolar magnetopause crossing, Phys. Rev. Lett. 89 (2002) 015002/1-4.

[151] A. Runov, R. Nakamura, W. Baumjohann, T.L. Zhang, M. Volwerk, H.-U. Eichelberger, A. Balogh, Cluster observation of a bifurcated current sheet, Geophys. Res. Lett. 30 (2003) 1036.

[152] S.K. Solanki, A. Lagg, J. Woch, N. Krupp, M. Collados Three-dimensional magnetic field topology in a region of solar coronal heating, Nature (London) 425 (2003) 692-695.

[153] See many articles in the Special Issue: Fourth International Workshop on Nonlinear Waves \& Chaos in Space Plasmas, Nonlinear Proc. Geophys. 10 (2002).

[154] P.K. Shukla, B. Eliasson, Complete theory for Langmuir envelope solitons in dusty plasmas, Phys. Plasmas 11 (2004) 584-592.

[155] P.K. Shukla, B. Eliasson, Localization of intense electromagnetic waves in a relativistically hot plasma, Phys. Rev. Lett. 94 (2005) 65002/1-4.

[156] M. Abramowitz, I.A. Stegun, Handbook of Mathematical Functions, Dover, New York, 1972.

[157] E. Isaacson, H.B. Keller, Analysis of Numerical Methods, Dover, New York, 1994.

[158] D.A. Mendis, M. Rosenberg, Cosmic dusty plasmas, Ann. Rev. Astron. Astrophys. 32 (1994) 419-463.

[159] F. Verheest, Waves in Space Dusty Plasmas, Kluwer Academic, Dordrecht, 2000.

[160] P.K. Shukla, A.A. Mamun, Introduction to Dusty Plasma Physics, Institute of Physics, Bristol, 2002.

[161] D.A. Mendis, Progress in the study of dusty plasmas, Plasma Sources Sci. Technol. 11 (2002) A219-A228.

[162] See for example, many articles in: M. Horányi, S. Robertson, B. Walch (Eds.), Physics of Dusty Plasmas, AIP, New York, 1998.

[163] J.P. Boeuf, C. Pusset, in: A. Bouchoule (Ed.), Dusty Plasmas, John Wiley \& Sons, New York, 1999.

[164] P.O. Dovner, A.I. Eriksson, R. Bostrom, B. Holback, Freja multiprobe observations of electrostatic solitary structures, Geophys. Res. Lett. 21 (1994) $1827-1830$.

[165] A.A. Mamun, B. Eliasson, P.K. Shukla, Three-dimensional electroacoustic solitary waves induced by ion holes in magnetized plasmas, Phys. Plasmas 11 (2004) 4535-4538.

[166] P.K. Shukla, A.A. Mamun, B. Eliasson, 3D electron-acoustic solitary waves introduced by phase space electron vortices in magnetized space plasmas, Geophys. Res. Lett. 31 (2004) L07803/1-4.

[167] V.L. Krasovsky, H. Matsumoto, Y. Omura, Effect of trapped-particle deficit and structure of localized electrostatic perturbations of different dimensionality, J. Geophys. Res. 109 (2004) A04217/1-9 doi: 10.1029/2003JA010198.

[168] Li-Jen Chen, David J. Thouless, Jian-Ming Tang, Bernstein-Greene-Kruskal solitary waves in three-dimensional magnetized plasma, Phys. Rev. E 69 (2004) 50 (055401/1-4).

[169] U. Wagner, M. Tatarakis, A. Gopal, F.N. Beg, E.L. Clark, A.E. Dangor, R.G. Evans, M.G. Haines, S.P.D. Mangles, P.A. Norreys, M.-S. Wei, M. Zepf, K. Krushelnick, Laboratory measurements of 0.7 GG magnetic fields generated during high-intensity laser interactions with dense plasmas, Phys. Rev. E 70 (2004) 026401/1-5.

[170] K.G. McClements, R.O. Dendy, R. Bingham, J.G. Kirk, L.O.C. Dury, Acceleration of cosmic ray electrons by ion-excited waves at quasiperpendicular shocks, Mon. Not. R. Astron. Soc. 291 (1997) 241-249.

[171] R.Z. Sagdeev, V.D. Shapiro, Influence of transverse magnetic field on Landau damping, JETP Lett. 17 (1973) 279-282 Pis'ma Zh Eksp. Teor. fiz. (1973) 389-394.

[172] J.M. Dawson, V.K. Decyk, .Huff Robert W, I. Jechart, T. Katsouleas, J.N. Leboeuf, B. Lembege, R.M. Martinez, Y. Ohsawa, S.T. Ratliff, Damping of large-amplitude plasma waves propagating perpendicular to the magnetic field, Phys. Rev. Lett. 50 (1983) $1455-1458$.

[173] T. Katsouleas, J.M. Dawson, Unlimited electron acceleration in laser-driven plasma waves, Phys. Rev. Lett. 51 (1983) $392-395$. 